\title{
Hirshfeld surface analysis of crystal packing in aza-aromatic
}

\section{picrate salts}

Eric J. Chan, ${ }^{\mathrm{a}, \#}$ Simon Grabowsky, ${ }^{\mathrm{a},}{ }^{*}$ Jack M. Harrowfield, ${ }^{\mathrm{a}, \ddagger}$ Ming Wen Shi, ${ }^{\mathrm{a}}$ Brian W. Skelton, ${ }^{\mathrm{a}, \dagger}$ Alexandre N. Sobolev ${ }^{\mathrm{a}}$ and Allan H. White ${ }^{\mathrm{a}}$

Single crystal X-ray structure determinations have previously been described for picrate salts of a variety of nitrogen bases. Herein these have been extended to encompass monoprotonated mono- and oligo-dentate cyclic nitrogen-donor ligand systems derived from pyridine, some via saturation (piperidine and morpholine), and others via lateral extension of the aromatic system (2,2'-bipyridine, 1,10-phenanthroline, 2,9-dimethyl-1,10-phenanthroline, bis(2pyridyl)amine, 2,2':6',2"-terpyridine, and 8-hydroxyquinoline). Hydrogen-bonding

\footnotetext{
a School of Chemistry and Biochemistry, M310, The University of Western Australia, Crawley, WA 6009, Australia;

*e-mail: simon.grabowsky@uwa.edu.au,Tel.: +61 (0)8 6488 3515, Fax: +61 (0)8 64881005

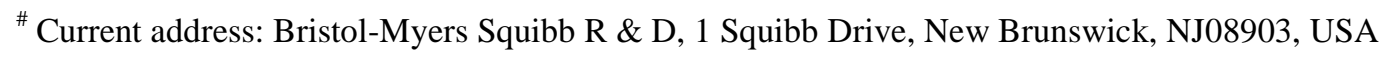

${ }^{\ddagger}$ Current address: Institut de Science et d'Ingenierie Supramoleculaires, Université Louis Pasteur, 8 allée Gaspard Mongé, 67083 Strasbourg, France

${ }^{\dagger}$ Current address: Centre for Microscopy, Characterisation and Analysis, M310, The University of Western Australia, Crawley, WA 6009, Australia
}

Electronic Supplementary Information (ESI) available: .CIF files for the structurally characterised compounds of

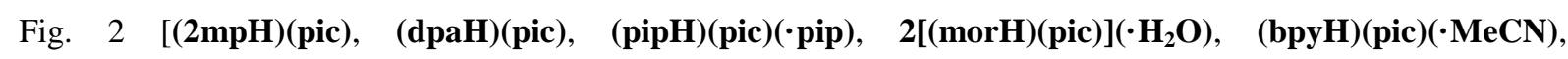
(tpyH)(pic), (phenH)(pic), (dmpH)(pic), (ohqH)(pic)] (CCDC: 606097-606105). Tables: saturated base nonhydrogen geometries (S1), pyridine-base ring non-hydrogen geometries (S2), phenanthroline non-hydrogen geometries (S3), quinoline-derived array non-hydrogen geometries (S4), non-hydrogen atom picrate anion geometries (S5), interspecies approaches and analysis of contact types for the compounds of Table 1 (S6, S7). Figures: Unit cell projections (S1), aromatic component overlap projections (S2), Hirshfeld surface projections (S3), for the compounds of Table 1. 
interactions are dominant determinants of the structures, complemented by or in competition with parallel stacking of anion and (aromatic) base planes. Furthermore, nitro...nitro, nitro... $\pi$ and phenoxy-O... $\pi$ inter-species contacts play a significant role in the crystal packing. It also appears that cation...anion interactions arising from $\mathrm{CH}$ (adjacent to $\mathrm{NH}$ )...o-nitro-O interactions are more important than the available secondary bifurcating component associated with any NH...o-nitro-O approach, resulting in many cases in a bidentate HN...CH base approach to an $\mathrm{O}(\mathrm{NO})$... O...(NO)O triadic array. The nature of the anion-cation interactions and their importance is explored using the Hirshfeld surface method. The precision of the structure determinations establishes the quinonoid form of the picrate to be a widespread contributor. Theoretical calculations on picric acid and the parent pyridiniumpicrate ion pair confirm the energetic favourability of the base-triad approach and the dominance of the quinonoid resonance form.

\section{Introduction}

The crystalline form of picric acid (2,4,6-trinitrophenol) (picH) ${ }^{1}$ presents an interesting demonstration of the multiple types of forces which may control the form of crystalline solids more generally, hydrogen-bonding ${ }^{2}$ and aromatic-aromatic interactions ${ }^{3}$ being two of the most familiar. These two interactions in particular appear to reach an interesting balance in the case of picric acid, the lattice of which is, at first sight, somewhat anomalous when compared with those of a wide variety of picrate derivatives. Thus, picric acid (Scheme 1), which has been subjected to extensive crystallographic studies (orthorhombic, $P c a 2_{1}$ ), ${ }^{1}$ has been perceived as showing 'herringbone' arrays of stoichiometric units in its lattice in which there are sets of molecules for which the mean ring planes are parallel, one of the necessary features of/prerequisites for 'stacking' (cf. Fig. 1). ${ }^{3,4}$ The projection down $a$ clearly displays the 

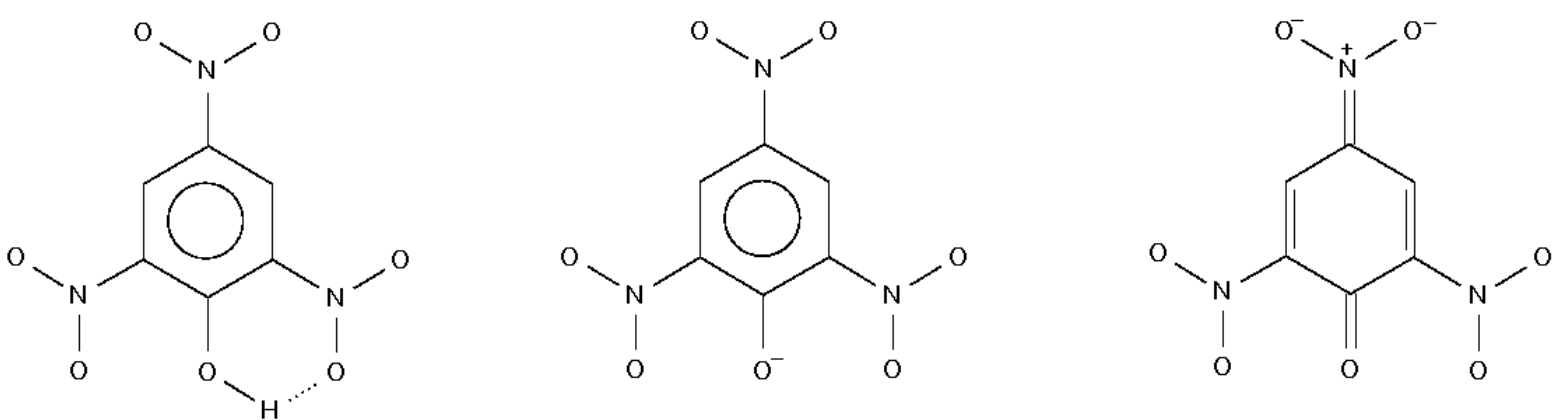

Scheme 1 The protonated and deprotonated forms of picric acid (picH), showing the most relevant Lewis (quinonoid) resonance structure (right). Nitro groups are represented schematically with single lines between the $\mathrm{N}-\mathrm{O}$ atoms to accommodate the various possible resonance forms.

'herring-bone' packing of molecules 1 , forming stacks of channels which lie along $a$, one such clearly seen in the projection down $c$. Molecules 2 also display 'herring-bone' packing, seen in the projection down $c$, the view into the channel being seen in the projection down $a$. However, instead of packing as infinite stacks, as molecules 1 do (separated by arrays of molecules 2) (see projection down $a$ ), the channels of molecule 2 pack pairwise side by side (see projection down $c$ ). The projection down $b$ shows dispositions of the two types of sheet comprised of the two different 'herring-bone' arrays generated by molecules 1,2 . Although these are associated with some short interatomic contacts, they are found only peripherally, involving nitro-group oxygen atoms, adjacent units showing no overlap in projection, perpendicular to the mean ring planes. Recent low-temperature studies ${ }^{1 \mathrm{f,g}}$ have secured the acidic hydrogen atom dispositions in each of the two molecules of the asymmetric unit as being disposed in intramolecular hydrogen bonds, contacting the oxygen atoms of adjacent $o$ nitro groups, as above. In contrast, the long-known adducts of picric acid with aromatic hydrocarbons - the 1:1 picric acid:benzene, naphthalene, anthracene, phenanthrene complexes being fundamental examples, ${ }^{5}$ well-characterized to the extent of demonstrating stacks of 
alternating acid/aromatic hydrocarbon entities - involve extensive overlap of their ring atoms, and entail contacts in the range 3.5 - $3.6 \AA$ between them. In the benzene:picric acid complex, ${ }^{5 a}$ the picric acid units lie in sheets with pairs confronting so as to suggest intermolecular hydrogen-bonding involving the phenolic and 2,6-nitro groups, seemingly giving the acidic protons a coordination number of three (hydrogen atoms unfortunately not being recorded in the structure determination). The structures of aromatic aza-

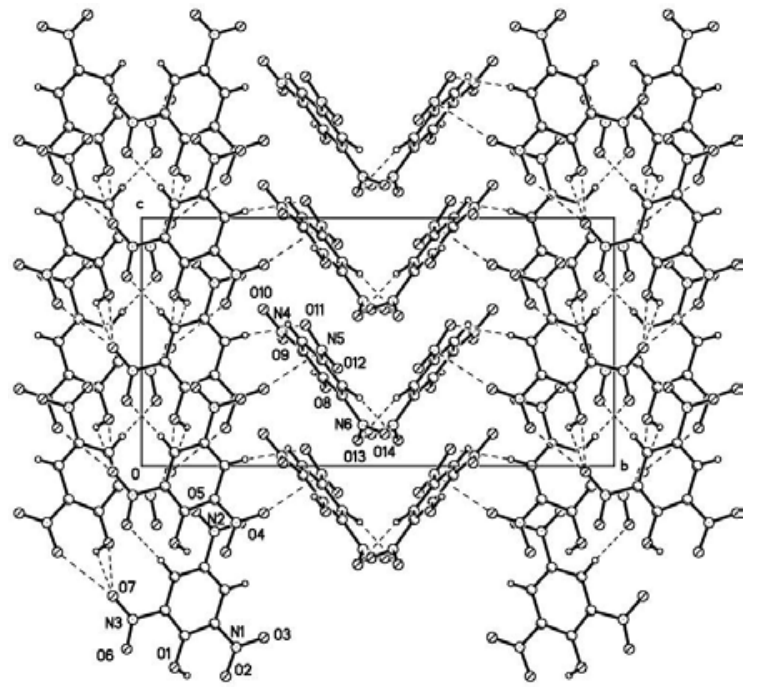

a)

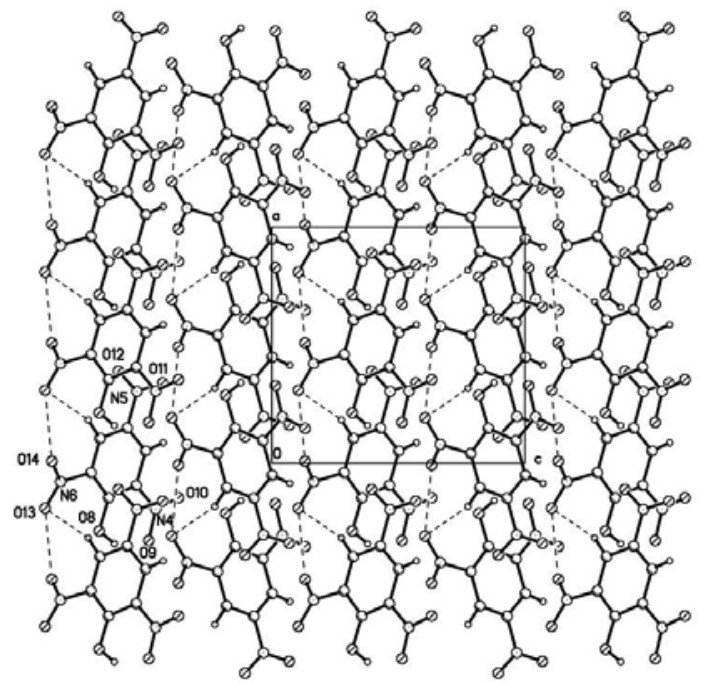

b)

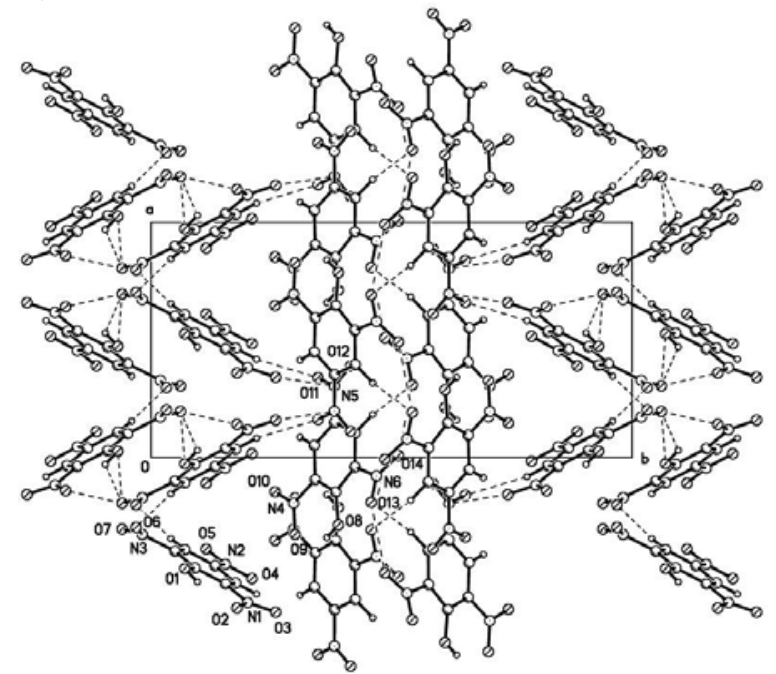

c)

Fig. 1 a) - c) Unit cell projections down $a, b, c$ respectively for picric acid (picH). The independent molecules 1 and 2 form two different hydrogen-bonded strands, with hydrogenbonding interactions being highlighted through dashed lines. 
cyclic picrates relevant to the present have been recorded in ref. ${ }^{6}$, and references therein (see later). When the proton of picric acid is replaced by a metal, true stacking interactions of picrate units become prominent, ${ }^{3 \mathrm{c}}$ albeit not universal, and hydrogen-bonding can also remain an influence when, for example, water of crystallisation is present. The structure of $\left[\mathrm{Fe}\left(\mathrm{OH}_{2}\right)_{6}\right](\mathrm{pic})_{2} \cdot 2 \mathrm{H}_{2} \mathrm{O}^{7}$ provides a clear illustration of this, but in organic salts classically used for derivatization and identification purposes, the work of ref. ${ }^{8}$ has identified a motif whereby, in hydrated forms, lattice water molecules may bridge protonated amine cation and picrate anion components ( $c$. Fig. 3 (e) below as an example of such a motif). The propensity of the highly acidic picric acid to form nicely crystalline salts has been particularly exploited in extending aromatic frameworks such as the above with pendant amine groups, as with salts of various anilines, ${ }^{9}$ and of substituted pyridines; ${ }^{8}$ it has also been utilized as a tool for exploring the manner in which variation in the $\mathrm{pK}$ of diverse bases, mostly peripherally substituted pyridines, ${ }^{1 \mathrm{~g}, 10}$ affects acid-base interaction and the packing thereof. In most of these arrays, the protonic hydrogen atom on the amine group has been perceived as interacting strongly with the phenolic oxygen atom of the picrate anion (Scheme 1), less so (chelate-wise) with an o-nitro oxygen atom, with further possibly more significant supportive interaction identified as possible between the latter and the adjacent C-H hydrogen. ${ }^{1 \mathrm{~g}}$ The parent pyridinium salt itself has been well-studied and shown to exist in two polymorphs: mono- and tri-clinic. $^{11}$

Although the above studies are extensive, there has been no systematic attempt thus far to explore more extended aromatic systems with embedded (rather than peripheral/exocyclic) basic nitrogen atoms. Thus, we anticipated that in salts of picric acid with aza-cyclic, in particular, aromatic, bases yet another determinant of equilibrium between the various types of forces might be pertinent. Several of the extended pyridine-derived aza-aromatic bases chosen in the present study (Scheme 2): 2,2'-bipyridine ('bpy'), 1,10-phenanthroline ('phen'), 
2,9-dimethyl-1,10-phenanthroline ('dmp'; 'neocuproin'), 2,2';6',2"-terpyridine ('tpy'), 2,2'dipyridylamine ('dpa') and 8-hydroxyquinoline ('ohq') are of particular interest because they are useful metal ion sequestrants involved in parallel studies of their complexation of metal picrates, ${ }^{12}$ and also because they are molecules capable of being involved, together with or in competition with picrate, in stacking and other aromatic or nitro-group interactions. ${ }^{3 a, 13} \mathrm{~A}$ parallel exploration of related systems has been made by examining salts formed with polyfunctional bases in which an aliphatic amine centre is the site of protonation, the present work also being a de-facto extension of detailed crystallographic studies of the salts formed by some of the present bases with other acids; ${ }^{14-18}$ we note also the recently reported study of competition between hydrogen-bonding and stacking interactions recorded for some picrate salts of some protonated linear $\alpha, \omega$-diamine systems. ${ }^{19}$

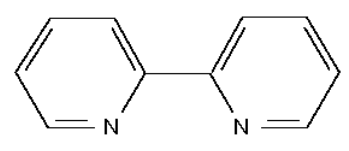

bpy<smiles>c1ccc(-c2cccc(-c3ncccn3)n2)nc1</smiles>

tpy<smiles>c1ccc(Nc2ccccn2)nc1</smiles>

dpa<smiles>Oc1cccc2cccnc12</smiles>

ohq

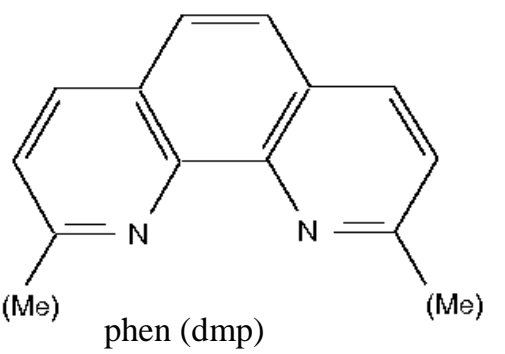

Scheme 2 The platform of extended pyridine-derived aza-aromatic bases structurally characterized in the present study as their picric acid salts. Note that for bpy, tpy different rotamers are possible, indicated in tpy by $(\mathrm{N})$.

In this work, developing from the pyridinium picrate polymorphs, we have also explored initially cyclic base arrays involving the related saturated piperidine ('pip') and morpholine ('mor'), followed by extension of the pyridine system by way of substitution, and 
then ring closure to the oligodentate forms noted above (Scheme 2); a number of related arrays have been the subject of study recently, some differing only in respect of solvation or hydration, or, as above, polymorphism, and we consider these aspects in conjunction with the present new results. All investigated compounds are summarised in Table $1 .^{1 \mathrm{~g}, 8,11 c, 20-30}$ In particular, in elucidating the structures of all of these compounds, Hirshfeld surface analysis methods ${ }^{31-33}$ have been employed to assist our better understanding of the diverse interactions possible between the various lattice components, particularly in respect of the relationship between polymorphs, ${ }^{34,35}$ and the different modes of overlap of the aromatic components; some extension to related Meisenheimer complexes ${ }^{30}$ is also explored.

Table 1 Picrate compounds referred to in the present study

$(\alpha)$ Picric acid (picH) $\left(\right.$ Pna $\left._{1}\right)$

$\left(\right.$ PICRAC13 $\left.^{1 \mathrm{~g}}\right)$

( $\beta$ ) Pyridinium and 2-substituted pyridinium (substituents other than pyridyl) picrates

(i,ii) Pyridinium picrate, (pyH)(pic) (mono- $\left(\boldsymbol{P 2} \mathbf{2}_{1} / \mathbf{c}\right)$ and tri-clinic $(\boldsymbol{P} \overline{\mathbf{1}})$ phases)

(PYRPIC02,03 ${ }^{11 \mathrm{c}}$ )

(iii) Pyridinium picrate : 1-naphthylamine (1:1), (pyH)(pic)(·naph) $\left(\mathbf{P 2} \mathbf{1}_{1} / \boldsymbol{a}\right)$ (PYNPCR ${ }^{20}$ )

(iv) 2-Methylpyridinium picrate, (2mpH)(pic) (x2) ( $\mathbf{P} \overline{\mathbf{1}})$ (this work)

(v) (2-Pyridinium)(2-pyridyl)amine picrate, $(\mathbf{d p a H})(\mathbf{p i c})\left(\boldsymbol{P 2} \mathbf{2}_{1} / \mathbf{c}\right)$ (this work)

$(\gamma)$ Aza-alicyclic base salts - Saturated derivatives of pyridinium picrate

(i) Piperidinium picrate, (pipH)(pic) ( $P \overline{\mathbf{1}})$ and

(VAZJAI ${ }^{21}$ )

(ii) its Piperidine solvate, (pipH)(pic)(·pip) $\left(\mathbf{P 2} \mathbf{2}_{1} / \mathbf{n}\right)$

(this work)

(iii) Morpholinium picrate, (morH)(pic) (x2) ( $\mathbf{P} \overline{\mathbf{1}})$ and (KOMTUC $^{22 a}$ )

(iv) its Hydrate, 2[(morH)(pic)] $\left(\cdot \mathbf{H}_{2} \mathbf{O}\right)(C 2 / c)$

(this work)

(ס) Bipyridinium picrates and derivative systems

(i) 2,2'-BipyridylH ${ }^{+}$picrate, (bpyH)(pic) ( $\left.\mathbf{P \overline { 1 }}\right)$

(UCOFUO $^{23}$ )

(ii) its Acetonitrile solvate, (bpyH)(pic)( $\cdot \mathbf{M e C N})(x 2)(\mathbf{P} \overline{\mathbf{1}})$

(this work)

(iii) 4,4'- BipyridylH ${ }_{2}^{2+}$ bis(picrate), (bpy' $\left.\mathbf{H}_{2}\right)(\mathbf{p i c})_{2}(\mathrm{x} 0.5)$ ( $\left.\boldsymbol{P} \overline{\mathbf{1}}\right)$ and

(KAMPIY $^{24}$ )

(iv) its Hydrate, $\left(\mathbf{b p y}^{\prime} \mathbf{H}_{2}\right)(\mathbf{p i c})_{2}\left(\cdot \mathbf{H}_{2} \mathbf{O}\right)(\mathbf{C} 2 / \mathbf{c})$ (UJOQUF ${ }^{25}$ )

(v) 2,2':6',2"-Terpyridinium picrate, (tpyH)(pic) ( $\boldsymbol{P} \overline{\mathbf{1}})$

(this work) 
(ع) 1,10-Phenanthrolinium picrate and derivative systems

(i) 1,10-Phenanthrolinium picrate, (phenH)(pic) $\left(\boldsymbol{P} \mathbf{2}_{\mathbf{1}} / \mathbf{c}\right)$

(this work)

(ii) Ammonium picrate : 1,10-phenanthroline (1:1), $\left(\mathbf{N H}_{4}\right)(\mathbf{p i c})(\cdot \mathbf{p h e n})\left(\boldsymbol{P} \mathbf{2}_{1} / \mathbf{c}\right)$

$\left(\right.$ AMPCPL $\left.^{26 a}\right)$

(iii) '1,10-Phenanthrolinium 4,4'-bipyridylH ${ }^{+}$1,10-phenanthroline' bis(picrate),

(INOSUZ ${ }^{27}$ )

' [(phenH)(bpy'H)(phen)]' (pic) ${ }_{2}$ (x0.5) ( P $\left.\overline{\mathbf{1}}\right)$

(iv) 2,9-Dimethyl-1,10-phenanthrolinium picrate, $(\mathbf{d m p H})(\mathbf{p i c})(\mathbf{P} \overline{\mathbf{1}})$

(this work)

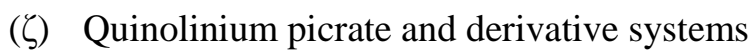

(i) Quinolinium picrate, (quinH)(pic) $\left(\mathbf{P 2} \mathbf{1}_{1} / \mathbf{c}\right)$

(UBEGAL ${ }^{8}$ )

(ii) iso-Quinolinium picrate, (iqH)(pic) $\left(\boldsymbol{P} 2_{1} / \boldsymbol{a}\right)$

(JUSRUK ${ }^{28}$ )

(iii) 2-Methylquinolinium picrate, $(\mathbf{2 m q H})(\mathbf{p i c})(\boldsymbol{P} \overline{\mathbf{1}})$

(VATTER $^{29}$ )

(iv) 8-Hydroxyquinolinium picrate, $(\mathbf{o h q H})(\mathbf{p i c})\left(\boldsymbol{P} \mathbf{2}_{1} / \mathbf{c}\right)$

(this work)

(v,vi) The 8-Hydroxyquinoline 'precursor', (oqpic) (C2/c)

$\left(\right.$ JOKTOS $\left.^{30}\right)$

and the 2-Hydroxymethylpyridine (omppic) Meisenheimer Salt $\left(\boldsymbol{P 2}_{\mathbf{1}} / \boldsymbol{n}\right)$

(JOKTIM $\left.^{30}\right)$

CCDC codes and references are given for those derivative of previous work.

\section{Experimental}

Syntheses. Although many amine picrate salts crystalline nicely from aqueous solution, the present compounds were prepared on a millimolar scale by adding the base to the correct molar ratio of picric acid (caution: picric acid and many of its salts are known explosives!) dissolved in ca $5 \mathrm{~mL}$ of acetonitrile or, if liquid at room-temperature, the corresponding base itself. The compounds are rather insoluble; to obtain crystals suitable for structure determinations they were carefully heated in small quantities of solvent until largely dissolved, the resulting partial solutions then being filtered into test tubes and the filtrates allowed to cool slowly by standing the tubes in hot water kept in a Dewar flask. Despite the possibility of thermal instability, perhaps manifest in explosive properties, all compounds melted without decomposition, enabling the measurement of melting points (Table 2). 
Structure determinations. CCD area-detector diffractometer data were measured (monochromatic Mo-K $\alpha$ radiation, $\lambda=0.7107_{3} \AA$, $\omega$-scans, $T$ ca $100 \mathrm{~K}$ ) yielding $N_{\mathrm{t}(\text { otal) }}$ reflections, these merging to $N$ unique ( $R_{\text {int }}$ cited) after 'empirical'/multiscan absorption correction, and being used in the full matrix least squares refinements on $F^{2}$. Anisotropic displacement parameters for the non-hydrogen atoms were refined, whereas hydrogen atom treatment followed a riding model except in respect of protonating species which were refined in $\left(x, y, z, U_{i s o}\right)$; reflection weights were $\left(\sigma^{2}\left(F_{\mathrm{o}}^{2}\right)+(\mathrm{a} P)^{2}(+\mathrm{b} P)\right)^{-1}\left(P=\left(F_{\mathrm{o}}^{2}+2 F_{\mathrm{c}}^{2}\right) / 3\right) . N_{\mathrm{o}}$ with $I>2 \sigma(I)$ were considered 'observed'. Neutral atom complex scattering factors were employed within the SHELXL97 program. ${ }^{36}$ Pertinent results are given in Tables 2-4 and the Figures of the main text and the Supporting Information. In the Figures, ellipsoids, where shown, are at the $50 \%$ probability amplitude displacement level for the non-hydrogen atoms, hydrogen atoms, where shown, having arbitrary radii of $0.1 \AA$. 
Table 2 Crystal/refinement data

\begin{tabular}{|c|c|c|c|c|c|c|c|c|c|}
\hline Cpd & $($ pipH)(pic)·pip & $2[(\mathrm{morH})(\mathrm{pic})] \cdot \mathrm{H}_{2} \mathrm{O}^{a}$ & $(2 \mathrm{mpH})(\mathrm{pic})^{b}$ & $\left(\right.$ bpyH)(pic) $\cdot \mathrm{MeCN}^{c}$ & (phenH)(pic) & (dmpH)(pic) & $(\mathrm{ohqH})(\mathrm{pic})^{d}$ & (dpaH)(pic) & (tpyH)(pic) \\
\hline Formula & $\mathrm{C}_{16} \mathrm{H}_{25} \mathrm{~N}_{5} \mathrm{O}_{7}$ & $\mathrm{C}_{20} \mathrm{H}_{26} \mathrm{~N}_{8} \mathrm{O}_{17}$ & $\mathrm{C}_{12} \mathrm{H}_{10} \mathrm{~N}_{4} \mathrm{O}_{7}$ & $\mathrm{C}_{18} \mathrm{H}_{14} \mathrm{~N}_{6} \mathrm{O}_{7}$ & $\mathrm{C}_{18} \mathrm{H}_{8} \mathrm{~N}_{5} \mathrm{O}_{7}$ & $\mathrm{C}_{20} \mathrm{H}_{15} \mathrm{~N}_{5} \mathrm{O}_{7}$ & $\mathrm{C}_{15} \mathrm{H}_{10} \mathrm{~N}_{4} \mathrm{O}_{8}$ & $\mathrm{C}_{16} \mathrm{H}_{12} \mathrm{~N}_{6} \mathrm{O}_{7}$ & $\mathrm{C}_{21} \mathrm{H}_{14} \mathrm{~N}_{6} \mathrm{O}_{7}$ \\
\hline$M_{\mathrm{r}}(\mathrm{Da})$ & 399.4 & 650.5 & 322.2 & 426.4 & 409.3 & 437.4 & 374.3 & 400.3 & 462.4 \\
\hline Cryst. syst. & Monoclinic & Monoclinic & Triclinic & Triclinic & Monoclinic & Triclinic & Monoclinic & Monoclinic & Monoclinic \\
\hline Space group & $P 2_{1} / n(\# 14)$ & $C 2 / c(\# 15)$ & $P 1(\# 2)$ & $P 1(\# 2)$ & $P 2_{1} / c(\# 14)$ & $P 1(\# 2)$ & $P 2_{1} / c(\# 14)$ & $P 2_{1} / C(\# 14)$ & $P 1(\# 2)$ \\
\hline$a(\AA)$ & 6.5862(5) & $17.8023(10)$ & $8.191(3)$ & $3.7068(10)$ & $13.509(5)$ & $8.2754(16)$ & $8.259(2)$ & $9.200(1)$ & $8.2700(5)$ \\
\hline$b(\AA)$ & 16.8950(13) & $15.2063(9)$ & 11.869(4) & 13.382(3) & 7.028(3) & 10.872(2) & $9.243(2)$ & $14.649(2)$ & $10.6206(6)$ \\
\hline$c(\AA)$ & $17.1440(14)$ & 20.3551(12) & 13.953(5) & 18.423(5) & 18.804(7) & $11.452(2)$ & 19.849(5) & 13.183(2) & $11.4612(7)$ \\
\hline$\alpha(\operatorname{deg})$ & & & $86.255(5)$ & 98.732(4) & & 98.064(4) & & & 82.126(1) \\
\hline$\beta$ (deg) & $99.306(2)$ & 102.331(1) & $82.754(6)$ & 93.699(4) & $98.116(6)$ & 104.517(3) & $91.282(4)$ & 108.918(2) & 86.883(1) \\
\hline$\gamma(\operatorname{deg})$ & & & $80.752(5)$ & $95.739(4)$ & & $102.449(4)$ & & & 71.856(1) \\
\hline Cell volume $\left(\AA^{3}\right)$ & 1882.5(3) & 5383.1(5) & 1326.8(8) & 895.8(4) & 1767.3(12) & 953.2(3) & $1514.9(7)$ & $1680.7(4)$ & $947.52(10)$ \\
\hline$D_{c}\left(\mathrm{~g} \mathrm{~cm}^{-3}\right)$ & $1.40_{9}$ & $1.60_{5}$ & $1.61_{3}$ & $1.58_{1}$ & $1.53_{8}$ & $1.52_{4}$ & $1.64_{1}$ & $1.58_{2}$ & $1.62_{1}$ \\
\hline$Z(\mathrm{fw})$ & 4 & 8 & 4 & 2 & 4 & 2 & 4 & 4 & 2 \\
\hline$\mu_{\mathrm{Mo}}\left(\mathrm{mm}^{-1}\right)$ & 0.11 & 0.14 & 0.14 & $0.12_{5}$ & $0.12_{2}$ & 0.12 & 0.14 & 0.13 & $0.12_{6}$ \\
\hline Spec. (mm) & $0.37,0.36,0.35$ & $0.32,0.27,0.25$ & $0.23,0.16,0.14$ & $0.22,0.17,0.15$ & $0.18,0.17,0.08$ & $0.42,0.36,0.18$ & $0.45,0.35,0.16$ & $0.40,0.36,0.31$ & $0.35,0.35,0.30$ \\
\hline$T_{\min } / T_{\max }$ (ratio) & 0.93 & 0.87 & 0.83 & 0.87 & 0.84 & 0.82 & 0.92 & 0.94 & 0.85 \\
\hline $2 \theta_{\max }(\mathrm{deg})$ & 75 & 67 & 58 & 58 & 57 & 60 & 58 & 67 & 75 \\
\hline$N_{\mathrm{t}}$ & 29353 & 37364 & 11660 & 8120 & 14321 & 11218 & 13846 & 22442 & 17639 \\
\hline$N\left(R_{\text {int }}\right)$ & $9889(0.037)$ & $9892(0.034)$ & $6180(0.038)$ & $4238(0.030)$ & $4149(0.059)$ & $5564(0.037)$ & $3773(0.022)$ & $6132(0.028)$ & $9451(0.020)$ \\
\hline$N_{0}(I>2 \sigma(I))$ & 6005 & 6865 & 4200 & 3104 & 2679 & 3260 & 2782 & 4482 & 6789 \\
\hline$R 1(I>2 \sigma(I))$ & 0.058 & 0.059 & 0.060 & 0.058 & 0.053 & 0.058 & 0.067 & 0.060 & 0.051 \\
\hline$w R 2$ (all data) & 0.166 & 0.166 & 0.164 & 0.20 & 0.142 & 0.168 & 0.133 & 0.171 & 0.149 \\
\hline a,b & $0.089,0.162$ & $0.091,2.6$ & 0.089, - & $0.097,0.81$ & 0.077, - & 0.095, - & $0.067,0.46$ & $0.097,0.52$ & $0.089,0.104$ \\
\hline$S$ & 0.99 & 1.01 & 1.08 & 1.11 & 1.05 & 1.00 & 1.03 & 1.04 & 1.03 \\
\hline$\left|\Delta \rho_{\max }\right|\left(\mathrm{e} \AA^{-3}\right)$ & 0.61 & 0.55 & 0.67 & 0.58 & 0.29 & 0.54 & 0.41 & 1.01 & 0.72 \\
\hline Solvent & Piperidine & Morpholine & $2 \mathrm{mp}$ & $\mathrm{MeCN}$ & $\mathrm{MeCN}$ & $\mathrm{MeCN}$ & $\mathrm{MeCN}$ & $\mathrm{MeCN}$ & $\mathrm{MeCN}$ \\
\hline
\end{tabular}




\section{Melting}

point $\left({ }^{\circ} \mathrm{C}\right)^{\mathrm{e}}$

${ }^{a} T$ was $170 \mathrm{~K} .{ }^{b}$ A recent determination (CCDC: $\mathbf{P E M C U G}{ }^{37}$ ) reports a room-temperature, single counter instrument study; one of the nitro group oxygen atoms is modelled as

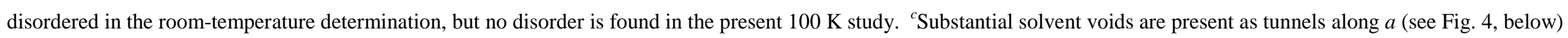

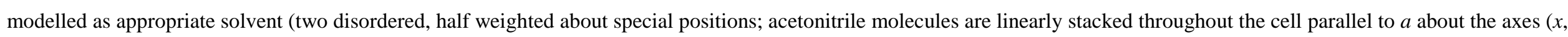
$1 / 2,1 / 2)$ and $(x, 1 / 2,0)$, one solvent molecule per axis per translation) with ill-defined atom locations using Platon 'SQUEEZE'; ${ }^{38}$ results are appended to the relevant .CIF file. ${ }^{d}$ The subject of previous determinations at room-temperature. ${ }^{39}$ The values given are averages over four different trials. First to third trials were performed on a Hotstage microscope (Reichert, Austria) fitted with a graduated mercury thermometer (range 120-350 degrees C with one degree increments). The fourth trial was performed on a Gallenkamp Melting point Apparatus fitted with a graduated mercury thermometer (range 10 - 360 degrees $\mathrm{C}$ with 2 degree increments). The samples were roughly crushed powders loosely fitted into glass capillaries (less than $1 \mathrm{mg}$ ). Repeats were performed on fresh samples. The averaged standard uncertainty of the determinations is about $6.5^{\circ} \mathrm{C}$. 
Hirshfeld surface and theoretical treatment. The Hirshfeld surface (HS) approach as implemented in the program CrystalExplorer ${ }^{31}$ has been employed. Directions and strengths of intermolecular interactions within the crystal have been mapped onto the HSs using the

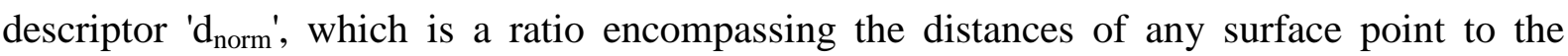
nearby interior $\left(\mathrm{d}_{\mathrm{i}}\right)$ and exterior $\left(\mathrm{d}_{\mathrm{e}}\right)$ atoms and the van der Waals radii of the atoms. ${ }^{32}$ Associated plots of $d_{i}$ vs $d_{e}$, referred to as 'fingerprint plots', are given, which are useful in identifying and comparing different kinds of interactions, ${ }^{33}$ particularly in similar crystal structures and polymorphs. ${ }^{34,35}$ For the generation of the fingerprint plots, and to allow a chemically meaningful analysis of HS properties, bond distances involving hydrogen atoms were reset to standard neutron diffraction values (C-H 1.083, N-H $1.009 \AA$ ). This entails elongation of the $\mathrm{C}, \mathrm{N}-\mathrm{H}$ bonds, impacting $\mathrm{H} . . . \mathrm{H}$ contacts in particular, so that $\mathrm{H} . . . \mathrm{H}$ contacts closer than the sum of their van der Waals radii (2.40 $\AA$ ) may be found. In the analysis of those published CIF files with SHELXL-type constraints on the bond distances, a contact distance offset was imposed in locating the closest individual $\mathrm{H}$...H contacts that are responsible for the features in the fingerprint plots of these compounds. The HSs were generated with the high resolution default with $\mathrm{d}_{\text {norm }}$ mapped over a colour scale ranging from -0.42 (red) - $1.6 \AA$ (blue), the fingerprint plots using the standard 0.6 - $2.6 \AA$ view of $\mathrm{d}_{\mathrm{e}} \mathrm{vs} \mathrm{d}_{\mathrm{i}}$. While the focus in the fingerprint plots is on the picrate ion throughout, a number of unusual features are found for some of the cations (for which the HSs are also shown), and we comment on these where appropriate. Crystal voids were calculated with CrystalExplorer based on either 0.002 au electron-density isosurfaces corresponding to smoothed van der Waals surfaces or 0.0003 au for the consideration of channels in the structures. ${ }^{31 \mathrm{c}}$

Geometry optimizations for the isolated picric acid molecule, the picrate anion, and a picrate-pyridinium contact pair were carried out at the B3LYP/6-311++G(2d,2p) level of theory using the program Gaussian $03 .{ }^{40}$ Frequency analyses were performed to ensure that the 
optimised structures were stationary points on the potential energy surface. Atomic charges were deduced from a subsequent natural population analysis being part of the NBO3.1 procedure implemented into Gaussian03. ${ }^{41,42}$

\section{Results and discussion}

Crystal structure determinations. A variety of salts, some solvated, formed by reaction between picric acid (picH) and several cyclic, mostly aromatic, nitrogen-bases, B, has been crystallized from various solvents as noted above, being, in general, golden-yellow/tan materials for which the results of the single crystal X-ray studies (Tables 2-4; S1-5) confirm/establish the stoichiometry/connectivity to be as given. All are of 1:1 acid:base stoichiometry, except in the case involving piperidine (pip) as the base, where the solvated species (pipH)(pic)(·pip) was obtained. Beyond this, the others, except the $\mathrm{morH}^{+}$and $b p y \mathrm{H}^{+}$ salts (see below), are unsolvated. In broad terms, the compounds are ionic $\mathrm{BH}^{+}$pic ${ }^{-}$with the lattice approaches between components as expected. The most conspicuous and persistent are those between the protonic hydrogen atoms and the phenoxide oxygen atoms. More distant approaches are possible to the o-nitro-oxygen atoms (Fig. 2), and also between the latter and nearby $\mathrm{C}-\mathrm{H}$ hydrogen atoms of aromatic rings, depending on the relative coplanarity of the various components. (The question of the validity of regarding these 'approaches' as 'interactions' is developed below, with the aid of Hirshfeld surface descriptions.). Table 4 presents detail for these approaches for the present and related compounds where relevant. Further obvious approaches may be noted arising from stacking/overlap of planar components; these will also be discussed below. A comprehensive suite of axial unit cell projections is presented in Figs. 1 (picric acid) and S1 together with the same for related compounds under consideration, as listed in Table 1; overlaps (mostly anion...anion) are 


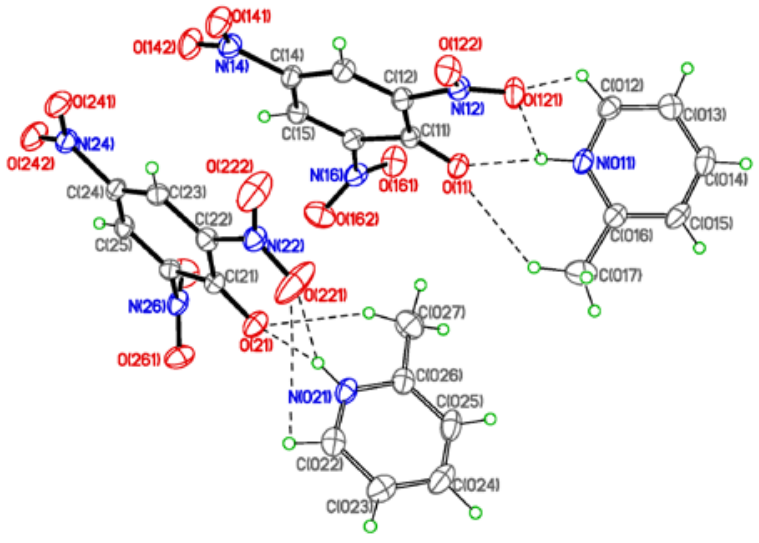

a) (ß)(iv) (2mpH)(pic)

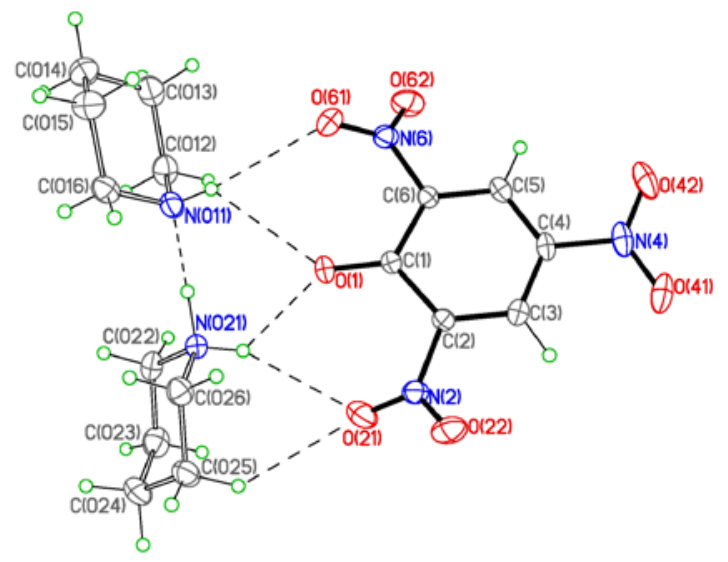

c) $(\gamma)(i i)(\mathbf{p i p H})(\mathbf{p i c})(\cdot \mathbf{p i p})$

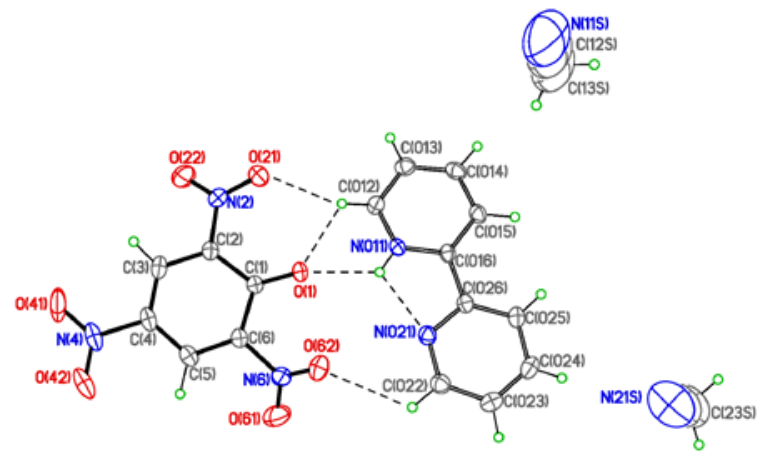

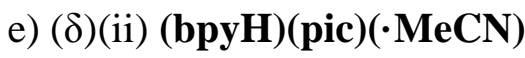

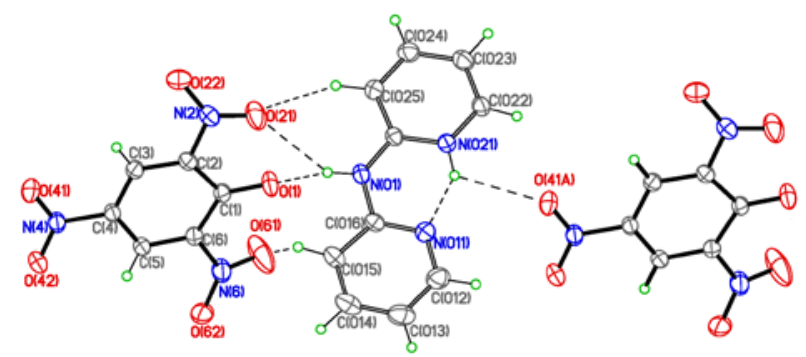

b) ( $\beta)(v)(\mathbf{d p a H})(\mathbf{p i c})$

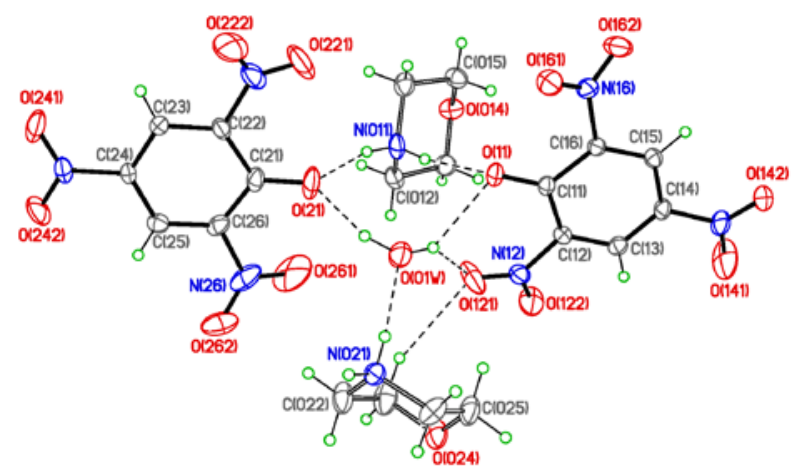

d) $(\gamma)\left(\right.$ iv) $2[(\mathbf{m o r H})(\mathbf{p i c})]\left(\cdot \mathbf{H}_{2} \mathbf{O}\right)$

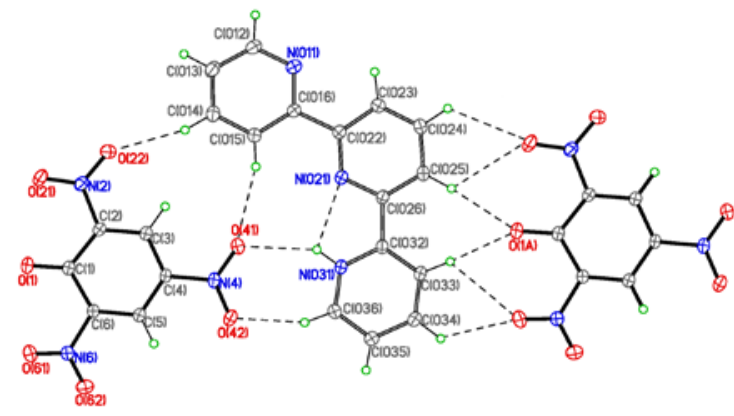

f) $(\delta)(v)($ tpyH)(pic) 


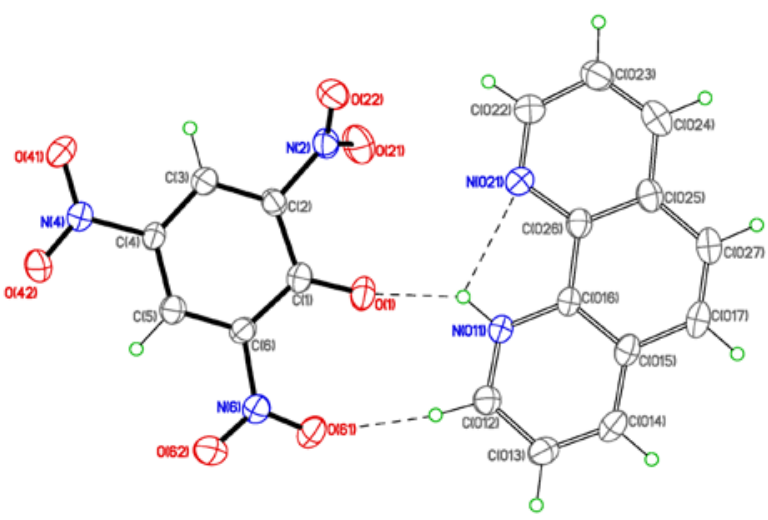

g) $(\varepsilon)(\mathrm{i})(\mathbf{p h e n H})(\mathbf{p i c})$

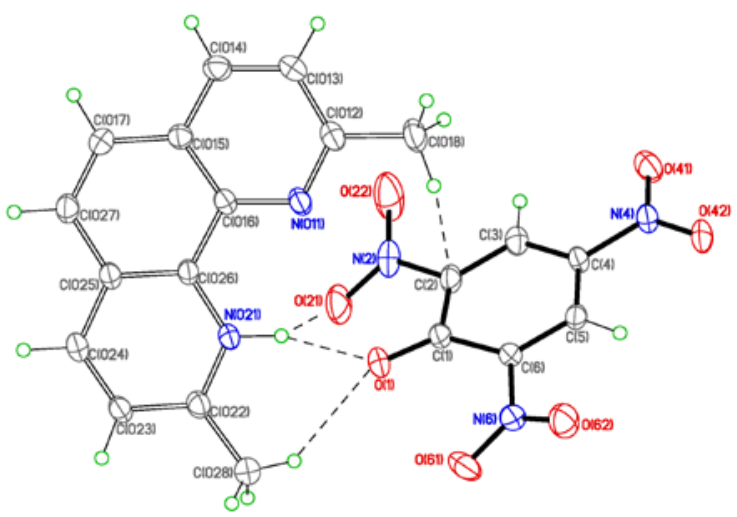

h) (E)(iv) (dmpH)(pic)

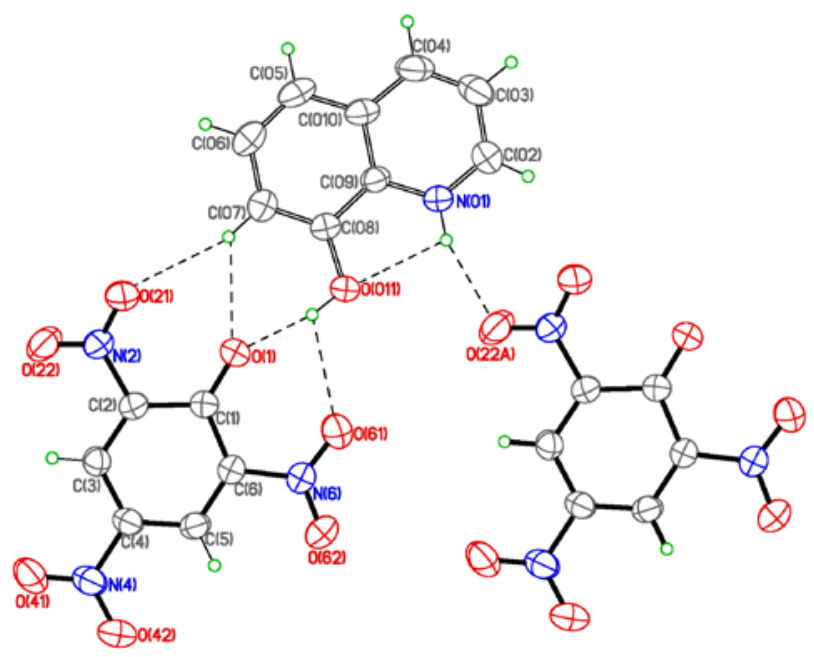

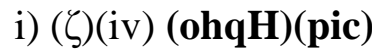

Fig. 2 a)-i) The asymmetric units (comprising the ion-pairs and any accompanying solvent) of the present determinations, showing the closer interspecies approaches (see also Table 2). Non-hydrogen atom labelling schemes are shown. Here and elsewhere, the lower case Greek alphabetical/Roman numeral codes follow the key to the compounds in the global scheme of Table 1.

shown in S2 and interatomic approaches in Tables 4 and S6, the latter using their 'local'/individual (i.e. CCDC) numbering schemes, as noted individually in the text. Hirshfeld surfaces and fingerprint plots for all compounds considered are comprehensively shown in the present text and Fig. S3. The former figures are projected normal to the planar aromatic component of the entity of interest, from both sides; the latter analyse the significant 
underlying contributing approaches (which, in respect of hydrogen atoms, may differ slightly from those recorded in Table S6 for reasons given in the Experimental Section).

\section{Intramolecular geometries and charges, experimental and theoretical. Detailed} experimental non-hydrogen geometries of the picrate components for the presently studied compounds are presented in Table S5. The descriptors are generally quite precise, being derivative of extensive $100 \mathrm{~K}$ data. Being pertinent to a diversity of compounds, these data highlight, perhaps more emphatically than any previous study, the considerable contribution of the quinonoid resonance form (see Scheme 1) across all of these species, a distribution in agreement with the interspecies interactions observed (see below); phenolic C-O distances are all $<1.271(2) \AA$, the shortest being 1.231(2) $\AA$, while the adjacent aromatic OC-C distances are $>1.437(2) \AA$ with $\mathrm{C}-\mathrm{CO}-\mathrm{C}$ all $<112.35(12)^{\circ}$. The $\mathrm{CNO}_{2}$ planes tend to lie parallel to the parent $\mathrm{OC}_{6} \mathrm{~N}_{3}$ plane, particularly that of the $p$-nitro group as befits the quinonoid descriptor (Table S5), but twisting out-of-plane may result in increased $\mathrm{O}$ (phenolic)...O(o-nitro) distances, particularly where there is no inhibition of that by 'chelation' of the cation protonic hydrogen atom between those atoms. In many cases, the $\mathrm{O}(o$-nitro $) \ldots \mathrm{O}$ (phenoxide)...O $(o-$ nitro) triad is an important motif, confronting the protonated amine-nitrogen and one or two adjacent $\mathrm{C}-\mathrm{H}$ hydrogen atoms; a more detailed discussion on the fundamental anion-cation interaction is presented in the next section.

Among the geometrical parameters of the aromatic base components (Tables S2-4), the usual variations are observed, with the angle at the nitrogen atom considerably increased (from $\mathrm{ca} 117$ to $123^{\circ}$ ) on protonation (the lone pair becoming dispersed as a bonding pair), and the associated N-C distances being essentially unchanged ( $c a 1.35 \AA$ ), as also the other intra-ring angles. Where there is a 2- or 6-substituent on the ring, the associated intra-ring angle is diminished in the protonated cases, enlarged in the others. The exocyclic angles are 
unequal, $\mathrm{N}(\mathrm{ar})-\mathrm{C}(\mathrm{ar})-\mathrm{C} / \mathrm{N}(\mathrm{exo})$ being less than $\mathrm{C}(\mathrm{ar})-\mathrm{C}(\mathrm{ar})-\mathrm{C} / \mathrm{N}(\mathrm{exo})$, perhaps a consequence of chelation of the relevant hydrogen atoms, being associated with diminished O...O distances (globally recorded in Table S6).

In addition, theoretical geometries and charges for gaseous picric acid and the picrate ion, derived as described above, together with their interaction with prototypical pyridine, are included in Table 3 and Scheme 3. A number of earlier theoretical calculations employing isolated-molecule geometry optimisation techniques have been reported for picric acid, ${ }^{43}$ and the picrate anion in various salts, ${ }^{44}$ one of the more recent ${ }^{43 a}$ also discussing calculations for the isolated picrate ion. The few distances and angles recorded therein and the charge distribution are harmonious with our present results, confirming the impact of the quinonoid contribution on the overall Lewis structure. An interesting divergence, however, is found in the twists of the pair of the $\mathrm{O}$-nitro groups, the $\mathrm{O}-\mathrm{N}-\mathrm{C}-\mathrm{C}$ torsions being recorded as 29.3, $25.5^{\circ}$, similar in magnitude to the present, but of opposed rather than same sign, so that the anion symmetry is $m$ rather than our more intuitive 2 (Table 4). That report and others ${ }^{43,44}$ also record studies for contact ion-pair systems with cations, the models for alkali-metal salts therein being overtaken by contemporary experimental results ${ }^{45}$ of a rather different nature.

All the reported theoretical calculations on picric acid or the picrate anion are on considerably lower levels of theory than those presented in this study, wherein a more extensive basis set involving diffuse functions aimed at improving the reliability and accuracy of the geometrical parameters and atomic charges for, at least, the charged species has been employed.

The results of the present theoretical calculations for the picrate anion are generally harmonious with the experimental results (Table S5). The calculations for the isolated anion in the gas phase result in the emergence of two-fold symmetry, wherein the nitro groups ortho to the phenolic oxygen atom are cosynchronously twisted out of plane, presumably in 
Table 3 Non-hydrogen atom picrate and picric acid geometries derived from theoretical calculations

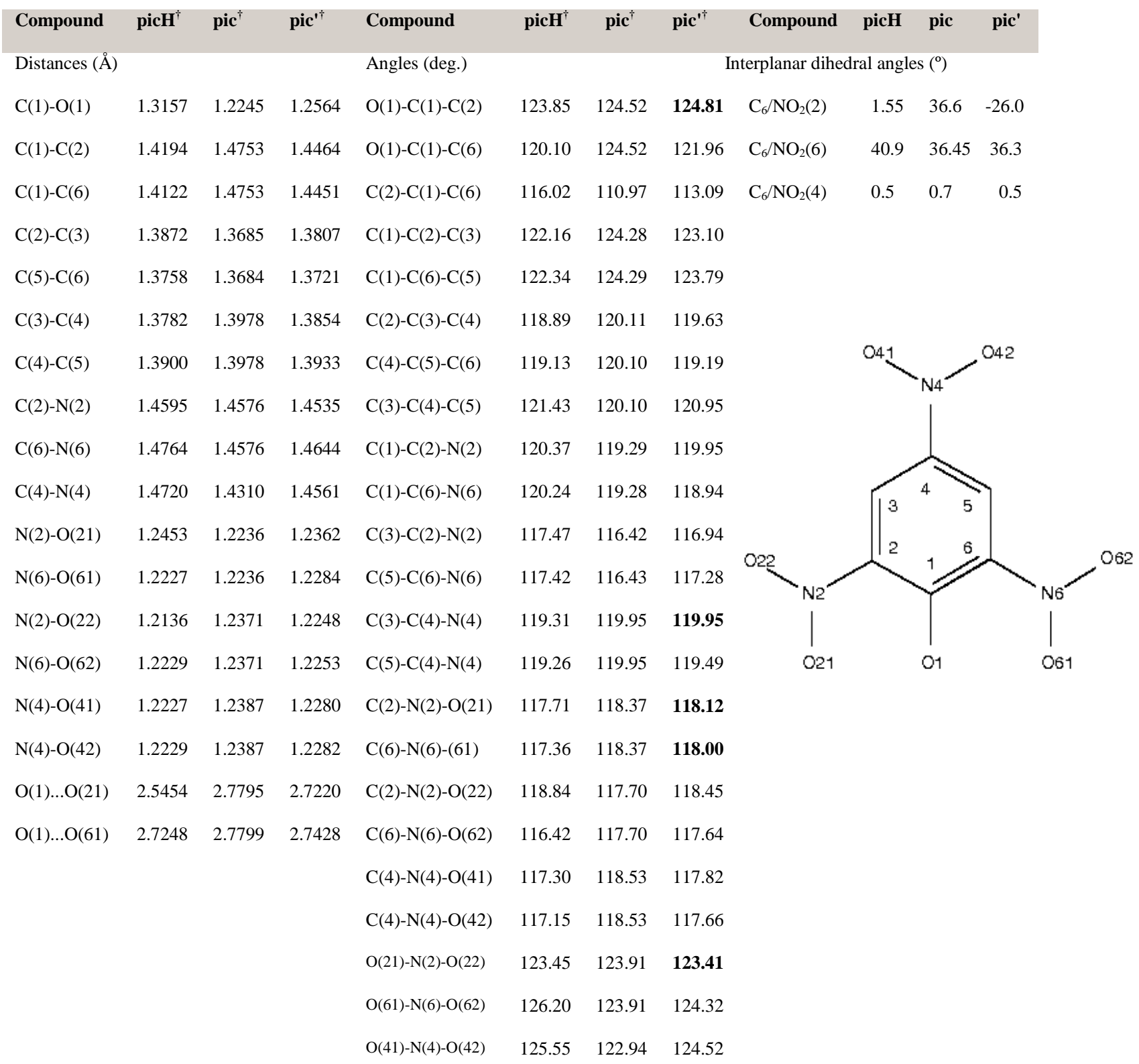

Values are given for the theoretical studies of picric acid (picH), the picrate anion in isolation (pic), and the picratepyridinium ion-pair (pic'). In the O...O distances, entries shown in bold are associated with chelation of a protonic hydrogen atom. The pictogram shows the ad-hoc numbering scheme used throughout this table.

avoidance of the ring substituents to either side. Quinonoid character, most emphatic in that half of the anion nearer the phenolic oxygen atom, is pronounced. There is a considerable asymmetry in the exocyclic angles at the pendant o-nitro group bonds, and a pronounced 
enlargement above the trigonal value of all $\mathrm{O}-\mathrm{N}-\mathrm{O}$ angles. The $\mathrm{C}-\mathrm{C}-\mathrm{C}$ angle opposite the phenolic oxygen pendant is greatly diminished below the trigonal value, in keeping with considerable double bond character in the C-O bond, and is compensated for by enlargement of the C-C-C angles opposite the o-nitro pendants. In the parent picric acid, there is an intramolecular hydrogen-bond between the phenolic hydrogen atom and the nearest oxygen atom of one of the $o$-nitro groups, resulting in the latter group lying coplanar with the aromatic ring. The associated $\mathrm{C}-\mathrm{O}$ and $\mathrm{N}-\mathrm{C}$ distances are lengthened, $c f$. the anion, the $\mathrm{N}-\mathrm{C}$ distance also, $c f$. its counterpart distance to the other $o$-nitro group, which, unaffected by the protonating hydrogen atom, remains twisted out of plane, presumably in consequence of steric interaction with the adjacent ring substituent atoms. The C-N distance at the $p$-position of the ring is also lengthened, $c f$. its anion counterpart with its quinonoid component. A considerable difference is now found between the $\mathrm{N}-\mathrm{O}$ distances of the $o$-nitro group which interacts with the protonic hydrogen, the proximate distance being elongated. The C-C distances to either side of the C-O bond are considerably shortened. In short, there are considerable changes in that part of the molecule associated with the $\mathrm{C}(1,2,6)$ atoms of the aromatic ring, less so in the (C3-5) component, except for the pendant at the p-position. Within the aromatic ring, charges at the $1,3,5$ positions in the anion are significant $(+0.43,2 \mathrm{x}-0.15 \mathrm{e})$, with a rather similar distribution in the acid. About the molecular peripheries significant positive charges are found at the hydrogen atoms, more so at the nitrogen atoms, all oxygen atoms having significant negative charges, with distributions throughout both species being rather similar. Here, and in the experimental data, those $o$-nitro groups not hydrogen-bonded to the phenolic groups are twisted considerably out of plane, despite the opposed charges of the adjacent hydrogen atoms. About the molecular peripheries of the acid, O...H...O distance strings are: 2.35(2); 2.47(2); 2.40(2) (x2) (mol.1) and 2.41(3), 2.41(3); 2.38(2), 2.35(2) $\AA$ (mol.2). 

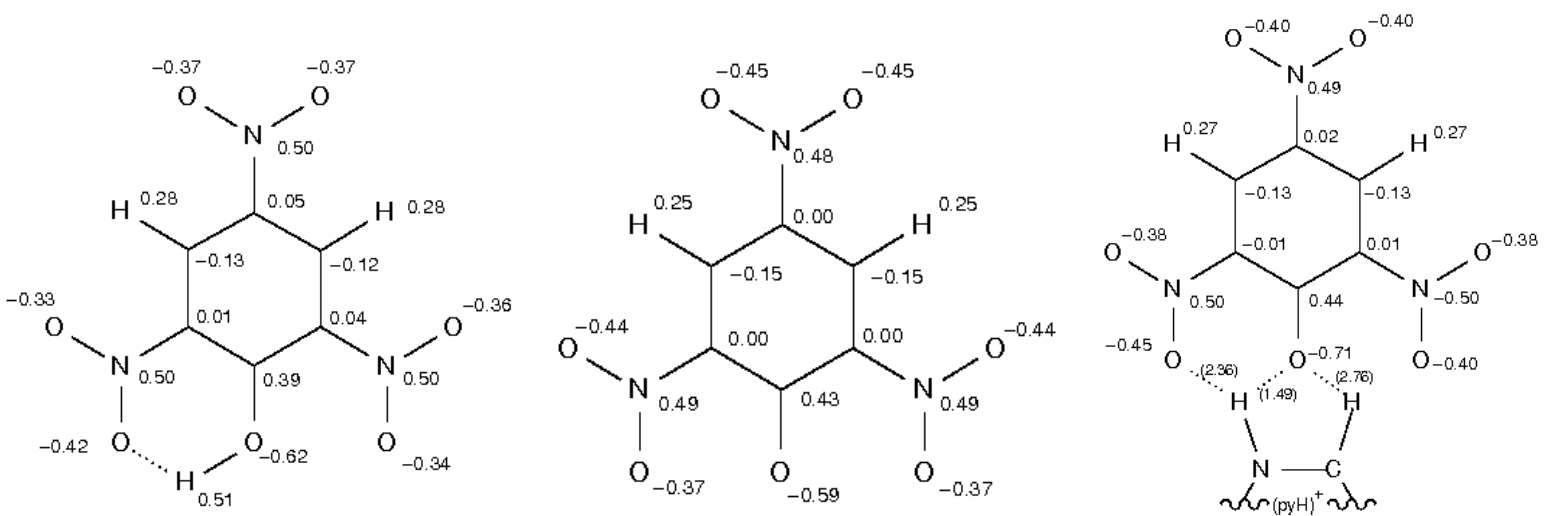

Scheme 3 Atom charges derivative of the theoretical calculations for the isolated picric acid (picH), left, an isolated picrate species (pic), center, and a picrate-pyridinium ion-pair (pic'), right (the latter also showing resulting H...O approaches $(\AA)$ ).

\section{Intermolecular geometries - fundamental anion-cation interactions and Hirshfeld}

surfaces. In the previous section, intramolecular geometries (covalent bonds) and associated charges were discussed that allow conclusions about the predominant Lewis resonance structures. In this section, the experimental intermolecular geometries are discussed, mainly pertaining to the fundamental anion-cation base...phenoxy-O hydrogen bonding motif NH...O...HC. Several variations of this motif exist (Scheme 4), with the base $\mathrm{NH}$ and $\mathrm{CH}$ groups additionally interacting with nitro-oxygen atoms leading to interesting chelating situations vis-a-vis the picrate O...O...O triad. However, the fundamental central NH...O...HC motif is preserved in most cases (Scheme 4). Hirshfeld surface representations (Fig. 3 and Fig. S3) show that this fundamental interaction guided by the protonated base N-H...phenoxyO approach leads to quasi-coplanar arrangements of protonated base cation and picrate anion in almost all cases ( $c f$. also Fig. 2). The motif of chelation or indentation around the negatively charged phenoxy-oxygen and positively charged N-H group can be apprehended easily by reference to these Hirshfeld surfaces (deep red regions on those of the picrate and the base, respectively, e.g., Figs. 3a) and b) as well as c) and d)). 
Table 4 summarizes the distances relevant to the NH...O...HC motifs that are depicted in Scheme 4, additionally providing distances between the base $\mathrm{NH}$ and $\mathrm{CH}$ groups to the nitro$\mathrm{O}$ atoms $\left(\mathrm{O}^{\prime}\right)$ in the picrate as well as the covalent phenoxy C-O bond distances. The latter are between 1.32 to $1.33 \AA$ in picric acid (protonated), contracting to ca $1.25 \AA$ in the deprotonated case, supporting the notion of a quinonoid resonance structure for the picrate anion. The differences in the hydrogen-bonding pattern lead to phenoxy C-O differences of up to $0.03 \AA$ ((tpyH)(pic) compared to (phenH)(pic)). The principal N...O distances are in general between 2.6 and $2.8 \AA$, and thus shorter than the $\mathrm{N} . . . \mathrm{O}^{\prime}($ nitro) distances which range between 2.8 and $3.1 \AA$ A. The N...O'(nitro) contacts are in turn mostly shorter than the C...O and C...O'(nitro) contacts, the latter ranging between 3.0 and $3.4 \AA$. These values are as expected, with the principal $\mathrm{N}-\mathrm{H} . . . \mathrm{O}$ hydrogen bond between the pair of charged species being a notably close interaction.

Fig. 3 shows a representative selection of plots for the cations and anions, exemplifying the important NH...O...HC motif on molecular Hirshfeld surfaces. In (pyH)(pic) (Figs. 3 a) and b)), $\mathrm{NH}$ and $\mathrm{CH}$ bonds are adjacent in the base, so that only one additional nitro group can be involved in the chelating interaction. In (quinH)(pic) (Figs. 3 c) and d)), separation of one of the $\mathrm{CH}$ groups from the $\mathrm{NH}$ group by extension of the aza-aromatic system leads to interactions with both nitro groups (cf. the relevant entries $(\beta)(i)$ and $(\zeta)(i)$ in Scheme 4). Similarly, derivatization of the aza-aromatic systems by other extensions results in different relative locations of the functional groups in the cation with respect to each other, allowing rational explanations of the observed patterns in Scheme 4 and Fig. S3 for most cases.

Two less prototypical but interesting special cases are shown in Figs. 3 e) and f). In $2[(\mathbf{m o r H})(\mathbf{p i c})]\left(\cdot \mathbf{H}_{2} \mathbf{O}\right)$ (Fig. 3 e)), a doubly protonated nitrogen atom bridges two picrate entities on one side, while a water molecule bridges the same ones from the other side (shown in Fig. S3( $\gamma($ (iv)). This leads to a more complex hydrogen-bonding pattern (Scheme $4(\gamma)(\mathrm{iv})$ ). 
In contrast, in $\left(\mathbf{N H}_{\mathbf{4}}\right)(\mathbf{p i c})(\cdot \mathbf{p h e n})^{26}$ the ammonium cation and the neutral 1,10-phenanthroline molecule form a composite, enabling a chelating motif with the picrate O...O...O triad that is very similar to the prototypical ones, but involving several NH groups instead of an NH plus $\mathrm{CH}$ pair (Scheme $4(\varepsilon)(\mathrm{ii}))$. Other relevant features or peculiarities in the fundamental base...phenoxy-O interactions are discussed in the sections dealing with the individual compounds below.

The quasi-coplanar approaches of the aromatic picrate anions and aza-aromatic bases governed by the principal NH...O...HC interactions allow opportunities for $\pi$-stacking between the planar ring fragments as well as concomitant nitro...nitro (i.e., O...O, N...O, N...N) and nitro... $\pi$ (i.e., N...C, O...C, N...N, O...N) interactions. Both types of interactions are significant for most compounds and also visible on the Hirshfeld surfaces (Fig. S3). Figs. 7 a) and b) show examples of pronounced red markers on the Hirshfeld surfaces indicative of significant $\pi \ldots \pi$ interactions between picrate anions in (pipH)(pic)(·pip). (For further discussion, see section $(\gamma)$ (ii) below.) On the left side of the Hirshfeld surface in Fig. 3 e) (as one of many examples (Fig. S3)), several red spots mark peripheral nitro-guided interactions. Details about these interactions will be discussed with the help of fingerprint plots, a breakdown of the Hirshfeld surface plots of Figs. 3, 7 and S3 into two-dimensional graphs, as described in the next section. 
( $\beta$

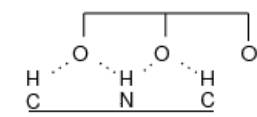

(i) $(\mathrm{pyH})(\mathrm{pic})$, monoclinic

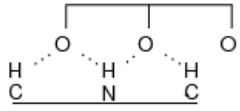

(ii) (pyH)(pic), triclinic

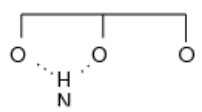

(iii) $(\mathrm{pyH})(\mathrm{pic})(\cdot$ naph

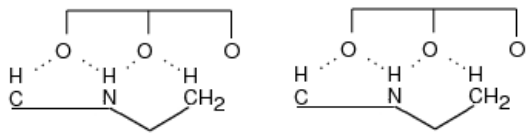

(iv) $2 \times(2 \mathrm{mpH})(\mathrm{pic})$

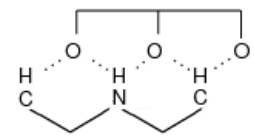

(v) $(\mathrm{dpaH})(\mathrm{pic})$

(v)

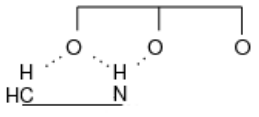

(i) $(\mathrm{pipH})(\mathrm{pic})$

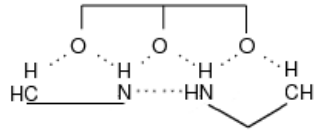

(ii) (pipH)(pic)(·pip)

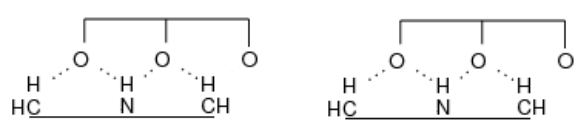

(iii) $2 x(m \circ r)(p i c)$

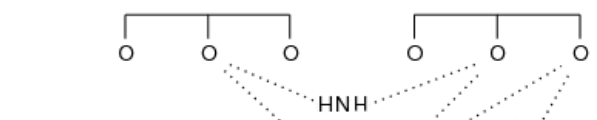

(iv) $2[(\mathrm{mor} H)(\mathrm{pic})]\left(\cdot \mathrm{H}_{2} \mathrm{O}\right)$

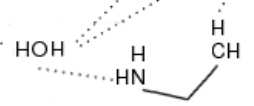

(6)

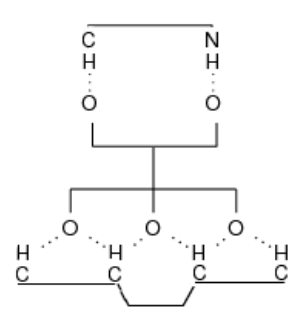

(i) (bpyH)(pic)
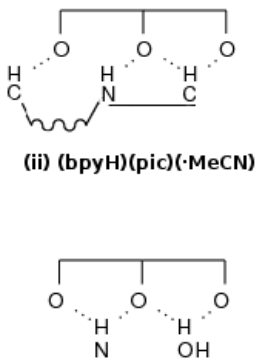

(iv) (bpy' $\left.\mathrm{H}_{2}\right)\left(\right.$ pic) $2\left(\mathrm{H}_{2} \mathrm{O}\right)$
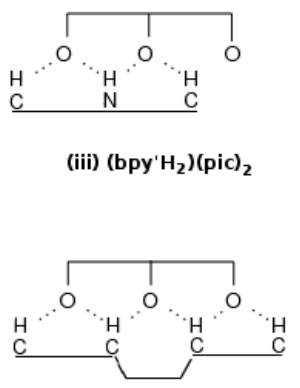

(v) (tpyH)(pic)

(ع)

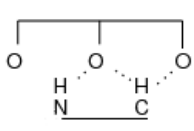

(i) (phenH)(pic)

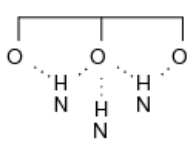

(ii) $\left(\mathrm{NH}_{4}\right)(\mathrm{pic})(\cdot \mathrm{phen})$

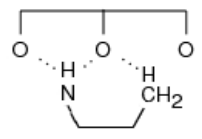

(iv) $(\mathrm{dmpH})(\mathrm{pic})$

(iii) $\left[(\right.$ phenH)(bpy' $\mathrm{H})($ phen) $]\left(\mathrm{pic}_{2}\right.$ has no picrate-O...base interactions.

(द)

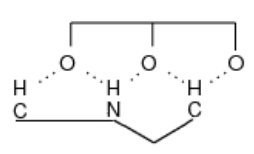

(i) $($ quinH)(pic)

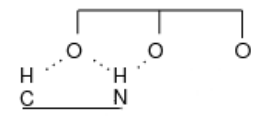

(ii) (iqH)(pic)

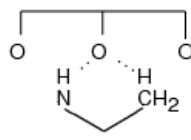

(iii) $(2 \mathrm{mqH})($ pic)

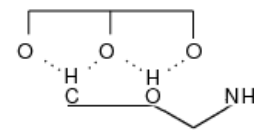

(iv) (ohqH)(pic)

Scheme 4 Schematic representation of the fundamental anion-cation interactions throughout the groups $(\beta-\zeta)$. 


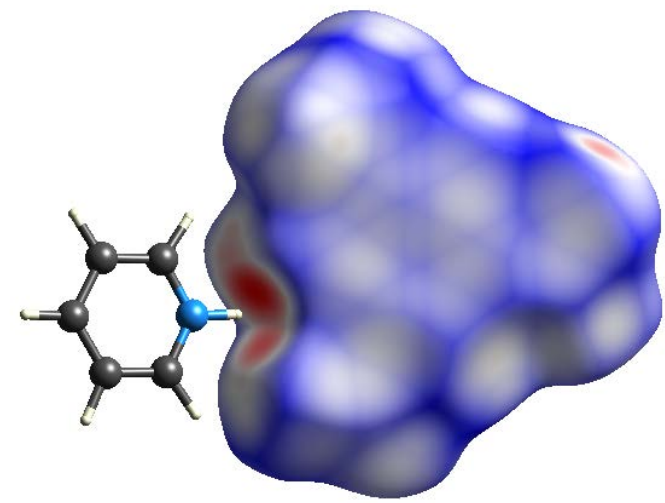

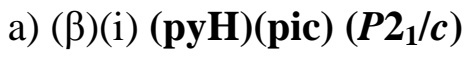

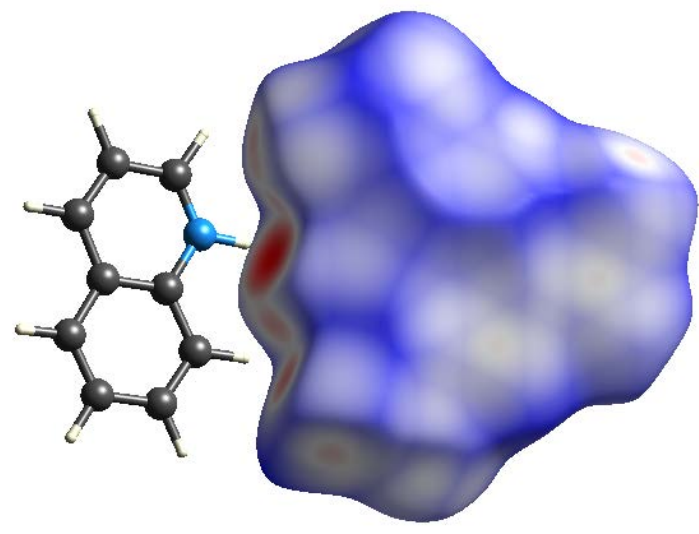

c) (ל)(i) (quinH)(pic)

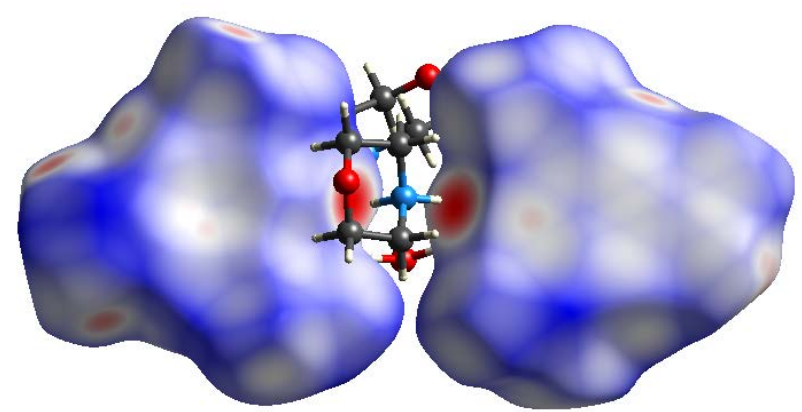

e) $(\gamma)\left(\right.$ iv) $2[($ morH $)($ pic $)]\left(\cdot \mathbf{H}_{2} \mathbf{O}\right)$

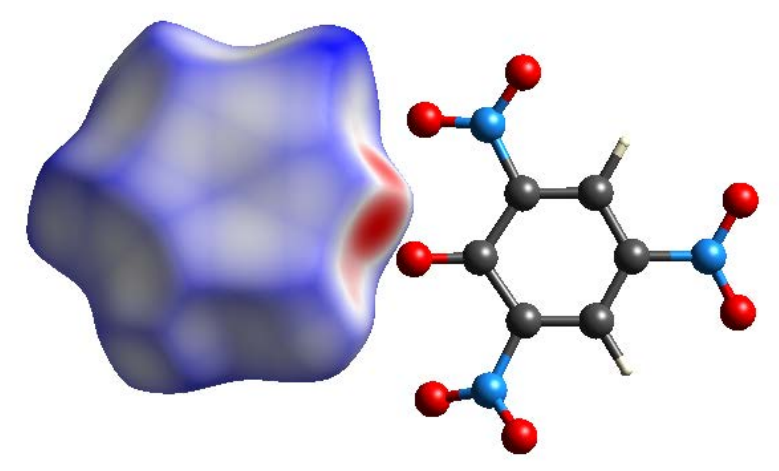

b) ( $\beta)(\mathrm{i})(\mathbf{p y H})(\mathbf{p i c})\left(P 2_{1} / c\right)$

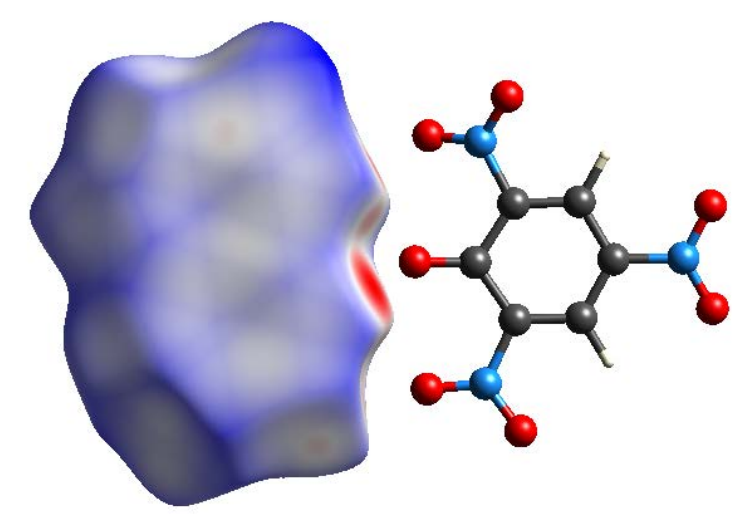

d) (ל)(i) (quinH)(pic)

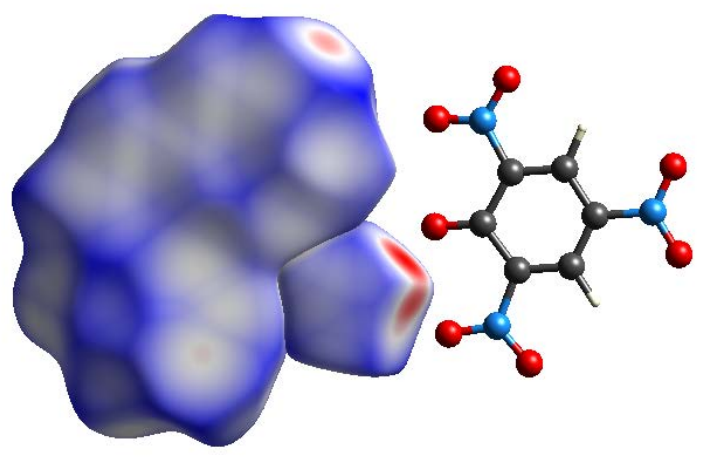

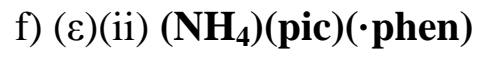

Fig. 3 a)-f) Hirshfeld surface representations for a representative selection of anion-cation ('solvent) clusters, with the property $\mathrm{d}_{\text {norm }}$ mapped, range -0.42 (red) to $1.6 \AA$ (blue) (see text). Note the composite (phen) $\left(\mathrm{NH}_{4}^{+}\right)$cation/base formation of $\mathrm{f}$ ). 
Table 4 Selected structural parameters for the base...phenoxy-O NH...O...HC approach, whereby O' are the $o$-nitro-O oxygen atoms, C-O are the phenoxy distances, and C...O(phenoxy) are shown in bold (cf. Scheme 4)

\begin{tabular}{|c|c|c|c|c|c|c|}
\hline Compd & C-O (A) & N...O (A) & N....' (A) & C.... $\left.\mathbf{O}^{(\cdot)}(\AA)\right)^{*}$ & REFCODE $^{\text {Ref }}$ & $\mathrm{C}_{6}(\mathrm{pic}) / \mathrm{NC}_{\mathrm{n}}(\mathrm{ar})\left(^{\circ}\right)$ \\
\hline (picH) (1) & $1.318(2)$ & - & - & - & PICRAC $^{1 \mathrm{~g}}$ & - \\
\hline (2) & $1.338(2)$ & - & - & - & & \\
\hline (pyH)(pic) (monoclinic) & $1.248(4)$ & $2.631(4)$ & $2.927(4)$ & 2.964(4), 3.179(4) & PYRPIC02 $^{11 c}$ & 18.69(9) \\
\hline (triclinic) & $1.241(6)$ & $2.666(5)$ & $2.875(6)$ & 3.061(6), 3.089(6) & PYRPIC03 $^{11 \mathrm{c}}$ & $9.9(2)$ \\
\hline (рyH)(pic)(·naph) & $1.240(7)$ & $2.694(7)$ & $2.811(7)$ & - & PYNPCR $^{20}$ & 67.11(3) \\
\hline (2mpH)(pic) (1) & $1.256(3)$ & $2.685(2)$ & $2.770(3)$ & 3.531(3), 3.102(3) & this work & 78.42(9) \\
\hline (2) & $1.258(3)$ & $2.664(2)$ & $2.756(3)$ & 3.352(3), 3.341(3) & & $77.00(9)$ \\
\hline (dpaH)(pic) & $1.247(2)$ & $2.755(2)$ & $3.121(2)$ & 3.424(2), 3.161(2), 3.448(2) & this work & 28.23(5) \\
\hline (pipH)(pic) & $1.243(3)$ & $2.767(2)$ & $2.996(3)$ & $3.437(3)$ & $\mathbf{V A Z J A I}^{21}$ & - \\
\hline (pipH)(pic)(·pip) & $1.2395(12)$ & 3.0507(13), 2.8143(12) & 3.3027(13), 3.3525(13) & 3.3891(17), 3.3946(15) & this work & - \\
\hline $2($ morH)(pic) (1) & $1.251(2)$ & $2.691(2)$ & $2.9248(13)$ & 3.278(2), 3.131(2) & KOMTUC $^{22 a}$ & - \\
\hline (2) & $1.2489(13)$ & $2.6837(14)$ & $2.923(2)$ & 3.280(2), 3.131(2) & & \\
\hline $2[(\operatorname{morH})(\mathrm{pic})]\left(\cdot \mathrm{H}_{2} \mathrm{O}\right)(1)$ & $1.261(2)$ & $2.808(2)$ & - & - & this work & - \\
\hline (2) & $1.261(2)$ & $2.705(2)$ & - & 3.554(5) (see text) & & \\
\hline (bpyH)(pic) & $1.243(2)$ & $*$ & $3.204(2) *$ & $\begin{array}{l}3.288(2), 3.325(2), 3.028(2) \\
3.147(2), 3.282(2), 3.323(2)\end{array}$ & $\mathbf{U C O F U O}^{23}$ & $16.74(6)$ \\
\hline (bpyH)(pic)(·MeCN) & $1.246(3)$ & 2.781(3) & - & 2.980(3), 3.271(4), 3.452(3) & this work & $10.78(7)$ \\
\hline$\left(\right.$ bpy' $\left.H_{2}\right)(\text { pic })_{2}$ & $1.250(2)$ & $2.647(2)$ & $3.097(3)$ & 3.162(2), 3.372(3), 3.414(2) & KAMPIY $^{24}$ & $51.29(5)$ \\
\hline
\end{tabular}




\begin{tabular}{|c|c|c|c|c|c|c|}
\hline$\left(\right.$ bpy' $\left.^{\prime} \mathrm{H}_{2}\right)(\mathbf{p i c})_{2}\left(\cdot \mathrm{H}_{2} \mathrm{O}\right)$ & $1.252(2)$ & $2.708(3)$ & $2.769(3)$ & - & UJOQUF $^{25}$ & $77.23(7)$ \\
\hline (tpyH)(pic) & $1.236(2)$ & $\dagger$ & $\dagger$ & $\dagger$ & this work & 11.94(3) \\
\hline (phenH)(pic) & $1.269(3)$ & $2.706(2)$ & - & 3.083(3), 3.324(3) & this work & $31.08(5)$ \\
\hline \multirow[t]{2}{*}{$\left(\mathrm{NH}_{4}\right)($ pic)(·phen) } & $1.250(3)$ & 2.834(3), 2.834(2), & 2.851(3), 3.080(4) & - & AMPCPL $^{26}$ & $0.90(7)$ \\
\hline & & 2.991(3) & & & & \\
\hline$\left[(\right.$ phenH $)(\text { bpy'H)(phen)](pic })_{2}$ & $1.238(3)$ & - & - & - & INOSUZ $^{27}$ & $\#$ \\
\hline$(\mathrm{dmpH})(\mathrm{pic})$ & $1.238(3)$ & $2.718(2)$ & $3.034(3)$ & 3.058(3) & this work & $47.65(5)$ \\
\hline (quinH)(pic) & $1.249(2)$ & $2.685(2)$ & $3.075(2)$ & 3.147(2), 3.081(2), 3.278(2) & UBEGAL $^{9}$ & $18.25(5)$ \\
\hline (iqH)(pic) & $1.250(5)$ & $2.590(4)$ & $3.086(5)$ & $3.077(6)$ & JUSRUK $^{28}$ & $3.17(12)$ \\
\hline (2mqH)(pic) & $1.243(3)$ & $2.749(3)$ & $2.857(3)$ & $3.443(4)$ & VATTER $^{29}$ & $84.21(10)$ \\
\hline (ohqH)(pic) & $1.234(2)$ & - & - & 3.081(2), 3.413(2) & this work & $4.14(5)$ \\
\hline (oqpic) & $1.353(5)$ & - & - & - & JOKTOS $^{30}$ & $65.1(2)^{\S}$ \\
\hline
\end{tabular}

* Nitro-O(4,5) are approached by $\mathrm{NH}(1), \mathrm{CH}(5)$ of the asymmetric unit at $\mathrm{N}(1) \ldots \mathrm{O}(5) 3.204(3) \AA, \mathrm{C}(5) \ldots \mathrm{O}(4) 3.284(3) \AA$; at the other end of the anion, the triad $\mathrm{O}(2,1,7)$ are approached by $\mathrm{CH}(3,2,7,8)(1+x, 1+y, z)$ as shown in Scheme 4, the six associated $\mathrm{C}(\mathrm{H}) \ldots \mathrm{O}$ distances ranged closely between 3.028(2)-3.325(2) $\AA$. † The primary array is similar to that for (bpyH)(pic): the picrate triad O(21,1,61) confronts four tpy CH groups $(24,25,33,34)(x, 1+y, z)$ as shown in Fig. 2 f) (O...C 3.110(2)-3.292(2) $\AA$ ) with $\mathrm{O}(41,42)$ of the asymmetric unit approaching $\mathrm{NH}(031), \mathrm{CH}(036)$ at 3.1083(13), 3.1666(14) $\AA$, with the greater complexity of $\mathrm{C}(014,015)$ of the additional pyridyl ring approached by O(22,41) at 3.2974(14), 3.3374(11) Å, nucleating a two-dimensional web-like array. \# pic/bpy; pic/phen interplanar dihedral angles are 4.61(9), 42.54(6); $47.11(8)^{\circ}{ }^{\S}{ }^{\S}$ Within JOKTIM (omppic) the intramolecular interplanar dihedral angle is 89.00(8) ${ }^{\circ}$. 
Fingerprint plots as global descriptors of crystal packings. Figs. 5 to 11 show the fingerprint plots of all independent picrate entities in the group of compounds summarised in Table 1 as obtained from a breakdown of the Hirshfeld surfaces mapped with the property $\mathrm{d}_{\text {norm}}$. They assist assignment of the crystal packing determinants; their interpretation is facilitated by the comprehensive list of all interspecies approaches and associated overlap projections given in the Supporting Information (Table S6). These figures comprise one of the most extensive comparisons of fingerprint plots presented for the same entity in different crystalline environments to date, with a large variety of shapes and features indicative of different types of crystal packing and contact types exhibited. The following sections will discuss in detail the packing of all compounds considered as expressed by their fingerprint plots and associated overlap pictures. Here, we present a summary of similarities and differences of the fingerprint plots.

In principle, the fingerprint plots only provide individual atom-atom distances. We have labelled the plots with an interpretation and assignment of the associated features in terms of the predominant intermolecular interaction types: hydrogen bonds, nitro...nitro, nitro... $\pi$ and $\pi . . . \pi$ interactions, and, hereafter, presume justification in referring to them as such. The prominent spikes in the plots are associated with closest contacts involving hydrogen atoms and can be attributed to $\mathrm{N}-\mathrm{H} . . \mathrm{O}, \mathrm{O}-\mathrm{H} . . . \mathrm{O}, \mathrm{C}-\mathrm{H} . . . \mathrm{O}, \mathrm{C}-\mathrm{H} . . . \mathrm{N}$, and $\mathrm{N}-\mathrm{H} . . . \mathrm{N}$ hydrogen bonds. In all cases, the dominant spike lies to the right (labelled $\boldsymbol{R}$ in Table S6), representing hydrogen bonds with the donor hydrogen atom being located within the picrate ion. Those for which the extension of the spike terminates with an atom-atom distance of $2.0 \AA$ or shorter, are associated with the major fundamental $\mathrm{N}-\mathrm{H}$...O ion-pair hydrogen-bond, as summarized in Table 4, with individual H...O distances becoming as short as $1.76 \AA$ (iso-quinolinium picrate, O...N 2.590(4) A). The only exception is 8-hydroxyquinolinium picrate in which the shortest hydrogen-bond is an unrepresentative $\mathrm{O}-\mathrm{H} . . . \mathrm{O}$ one. All other hydrogen-bonds exhibit 
distances of $\mathrm{H} . . . \mathrm{O} / \mathrm{H} . . . \mathrm{N}$ above $2.2 \AA$ (with a preponderance of C-H...O hydrogen-bonds), giving rise in each case to a more intensely coloured spike superimposed on the main spike (also denoted $\boldsymbol{R}$ in Table S6). In most fingerprint plots, secondary smaller spikes to the left of the main ones (labelled $\boldsymbol{L}$ in Table S6) arise from C-H...O hydrogen bonds with the donor hydrogen atom being located within the picrate ion. Occasionally, dihydrogen $(\mathrm{H} . . \mathrm{H})$ contacts are visible as a middle spike in the fingerprint plots (labelled $\boldsymbol{M}$ in Table S6). (Note that in the fingerprint plots, these dihydrogen contacts can appear as close as $2.2 \AA$ because they are affected most by the normalisation of $\mathrm{X}-\mathrm{H}$ distances to averaged neutron diffraction values within CrystalExplorer (see Experimental)). Peripheral spikes/features arise from CH... $\pi$ interactions. They are rather insignificant in the packing schemes of the discussed picrates, but are considered where obvious.

The central region of the fingerprint plots is dominated by contacts not involving hydrogen atoms. In most cases, $\pi \ldots \pi$ interactions arising from C...C, C...N, or N...N contacts can be distinguished from those involving the nitro groups. They are between approximately planar aromatic fragments, with inter-atomic distances ca $3.5 \AA$ or longer. The most intense central spots in the fingerprint plots (Figs. 5a) and 10a) as examples) occur at contact distances between 3.6 and $4.0 \AA$, showing that the preponderance of $\pi \ldots \pi$ contacts are in this rather long distance range. However, Table S6 only lists contacts shorter than 3.6 Å, labelled C. Interactions involving pairs of nitro groups, comprising N...O, O...O, or N...O contacts, ${ }^{46 a, b}$ respectively, are generally shorter than $3.5 \AA$, and therefore feature in a different (lower) central marker (also labelled $\boldsymbol{C}$ throughout Table S6), distinct from that for the $\pi \ldots \pi$ interactions, and also distinct from the central spikes, labelled $\boldsymbol{M}$. We note that intermolecular dichalcogen bonding in crystal packing has been described previously, ${ }^{46 a-c}$ but has only recently been established as a significant type of intermolecular interaction paralleling the more extensively studied dihalogen bonding. ${ }^{46 \mathrm{~d}}$ Certainly, in our study close contacts between 
pairs of electronegative atoms play a significant role in the crystal packing because of the dominance of the nitro groups, but we also find exceptionally close O...O contacts between the negatively charged phenoxy-O atoms of the picrate ion (e.g. 3.354(5) $\AA$ in the triclinic pyridinium picrate). Other types of contacts which occur in a similar distance range (i.e. 3.0 $3.5 \AA$ ), and which are thus to be found in the same central marker as the nitro...nitro interactions, are nitro...phenoxy-O (O...O and N...O), nitro... $\pi(\mathrm{O} \ldots \mathrm{C}, \mathrm{N} \ldots \mathrm{C}, \mathrm{O} \ldots \mathrm{N}, \mathrm{N} \ldots \mathrm{N})$, and phenoxy-O... $\pi(\mathrm{O} \ldots \mathrm{C}, \mathrm{O} \ldots \mathrm{N})$ interactions (all labelled $\boldsymbol{C}$ in Table S6). Some of the compounds discussed lack $\pi \ldots \pi$ interactions completely, but nitro...nitro as well as nitro... $\pi$ interactions are always present. Sometimes, the central regions showing $\pi \ldots \pi$ and nitro-involving interactions are indistinguishable when the contacts of the $\pi \ldots \pi$ interactions are exceptionally short and the nitro interactions themselves are not very pronounced.

The qualitative analysis of fingerprint plots emphasises individual contacts that are significantly shorter than all other inter-species contacts in the crystal packing as manifested through distinctive features arising in the plots. However, these prominent features do not necessarily represent the proportions of contacts of particular types in the global crystalpacking scheme. Fig. S7 gives these proportions of Hirshfeld-surface mediated contacts derived from a quantitative analysis of the fingerprint plots. In all of the salts of Table 1, hydrogen bonded (H...O) contacts amount to more than $50 \%$ of all contacts, which is remarkable since in molecular crystals $\mathrm{H} . . . \mathrm{H}$ contacts normally dominate, but here the latter are suppressed to below $10 \%$, consistent with the high melting points of the picrate salts (Table 2). Furthermore, the significance of nitro...nitro interactions as a central motif in the packing is confirmed by $\mathrm{N} . . . \mathrm{O}$ and $\mathrm{O} . . . \mathrm{O}$ contact contributions of between 10 and $20 \%$. For all compounds, the proportion of certain types of contacts correlates well with the presence or absence of corresponding central markers on the fingerprint plots, so that the qualitative analysis of the fingerprint plots (as employed in the following) is in fact representative in 
these cases. For example, nitro... $\pi(\mathrm{O} \ldots \mathrm{C}, \mathrm{N} \ldots \mathrm{C}, \mathrm{O} \ldots \mathrm{N}, \mathrm{N} \ldots \mathrm{N})$ interactions can be found with close to 30\% ((bpy' $\left.\mathbf{H}_{2}\right)(\mathbf{p i c})_{2}(\mathrm{x} 0.5)$, KAMPIY), or only 3\% ((pipH)(pic)(·pip)) (compare Fig. 6b) with Fig. 8c)). Also, the proportion of C...C contacts varies from 11.8 to $0.1 \%$ depending on the presence or absence of $\pi$-stacking geometries or a central marker in the fingerprint plots, respectively.

\section{(a) Picric acid}

Considering the crystal packing in the parent picric acid (picH; PICRAC ${ }^{1 \mathrm{~g}}$ ) (Fig. 1), two molecules comprise the asymmetric unit. The low-temperature studies exhibit no suggestion of disorder as suggested by the room-temperature determination of ref. ${ }^{1 b}$, not confirmed by the parallel studies of ref. ${ }^{1 c}$, which, however, noted further minor anomalies to be explored by neutron diffraction studies whose 'results will be published elsewhere'. The packing of the molecules has been discussed above. In neither of the arrays generated by molecules 1 and 2 are substantial overlaps found between the planar aromatic components; intermolecular contacts (Table S6( $\alpha)$ ) are to a considerable extent 'face-on' (as shown in the Hirshfeld surface projections below (Fig. S3a)), nearly all involving nitro-group oxygen atoms, and, in some cases, approaches between adjacent nitro-groups, some with $\mathrm{O} . . . \mathrm{O}$ as short as $2.807(3)$ and N...O as short as 2.771(2) Å. They are evident in a dispersed manner in the central regions of the fingerprint plots, in which any marker for $\pi . . \pi$ interactions is obviously absent. Most of the closest contacts are formed within the stacks of the same molecule ( 1 or 2 , respectively), and the few close contacts between species 1 and 2 are of the same type, e.g. one C-H...O hydrogen bond each. Thus, the fingerprint plots appear as quasi-symmetrical (Fig. 4), in clear contrast to the fingerprint plots of the picrate anions that interact with the protonated nitrogen bases. The donor hydroxyl O-H groups in the picric acid do not give rise to hydrogen bonds significantly closer than the C-H...O interactions, again in clear contrast to the remarkably 
short $\mathrm{N}-\mathrm{H} . . . \mathrm{O}$ hydrogen bonds found between the charged species in the salts as discussed below.

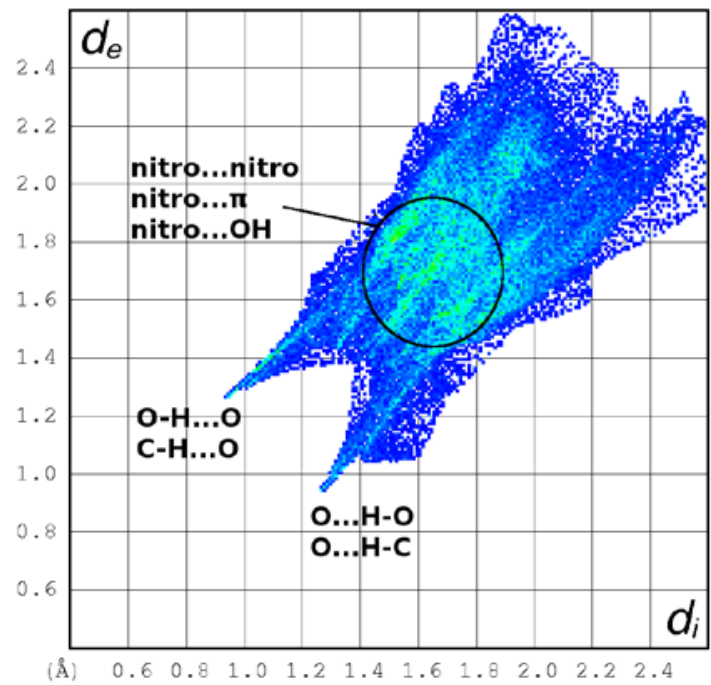

a) molecule 1

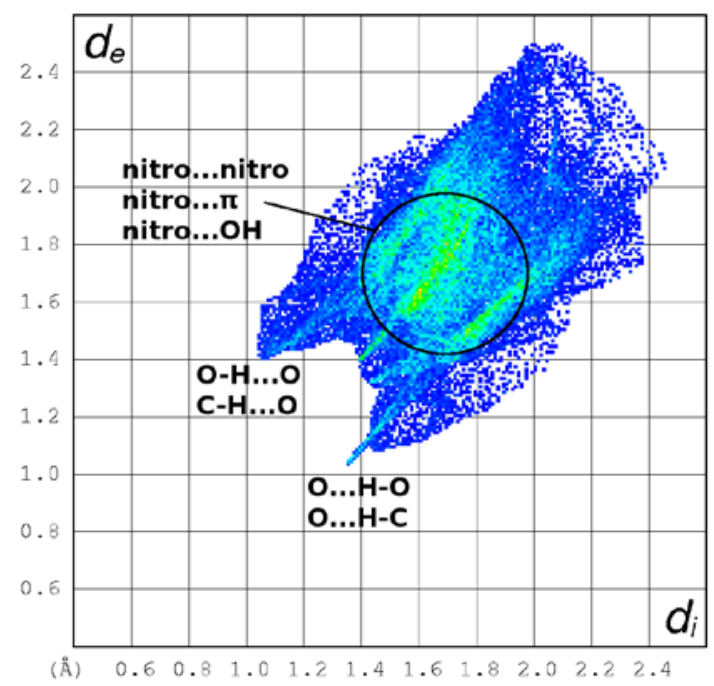

b) molecule 2

Fig. 4 Fingerprint plots of group ( $\alpha$ ): picric acid (picH) (after PICRAC13, ${ }^{1 \mathrm{~g}}$ two molecules), analyzed according to the various contacts displayed (see text).

\section{( $\beta$ ) Pyridinium and 2-substituted pyridinium (substituents other than pyridyl) picrates}

( $\beta$ ) (i,ii) Pyridinium picrate, mono- and tri-clinic. Beyond the parent acid, the salt pyridinium picrate (pyH)(pic) may be regarded as a useful 'substrate'/startingpoint/foundation for the present studies. Following earlier studies, ${ }^{11 a, b}$ one involving a report that it existed in two phases, ${ }^{11 \mathrm{e}}$ the structures of both of the latter (mono- and tri-clinic) have been resolved (PYRPIC02,03; Figs. S1( $\beta)(\mathrm{i}, \mathrm{ii})){ }^{11 \mathrm{c}}$ In keeping with many of the results of the more general study of (substituted) pyridinium picrate structures of ref. ${ }^{9}$ in which the substituent on the single aromatic ring may be structurally invasive, as is also the case in some of the adducts of ref. ${ }^{1 \mathrm{~g}}$, the dominant motif in both structures is a pyridinium picrate ion-pair (the asymmetric unit in each case). The acidic hydrogen atom is closely associated 
with the base (pyridine) nitrogen atom, but nevertheless interacting, quasi-three-coordinate, with a chelating approach by phenolic and adjacent $o$-nitro oxygen atoms. The nature of any interaction with the latter is a prominent feature to be considered throughout the remainder of the suite of compounds. We find it very frequently to be supported by $\mathrm{C}-\mathrm{H}$ interactions also, the latter having distances in this compound very similar in both forms (Tables S6( $\beta)(\mathrm{i}, \mathrm{ii})$ ). Schematic representations of the fundamental ion pair contacts can be found in Scheme 4 for all structurally defined compounds of the present type. The Hirshfeld surfaces shown in Figs. 3 a),b), provide a visual representation of the proton-chelating nature of the anion-cation contact, largely representative of the nature of this contact in the other compounds.

The dihedral angles between the quasi-parallel/coplanar pairs of aromatic planes of the ion-pair are also similar between the two phases $\left(18.69(9), 9.9(2)^{\circ}\right)$; the authors of ref. ${ }^{11 \mathrm{c}}$ regard the two phases as 'molecular crystals', differing in that (among other things), within the stacks observed in both, the ion pairs within each stack are parallel in the monoclinic form, antiparallel (inversion related) in the triclinic. In the monoclinic form (PYRPIC02), the unit cell projections clearly indicate the presence of columns of anions paired with cations side by side, albeit somewhat displaced and oblique to each other and to the unique axis, tending to a 'herringbone' aggregate, with the overlapping entities considerably displaced with respect to each other (Fig. S1( $\beta$ )(i)). Nonetheless, the dominant overlaps are those within the homogeneous stacks (Table S6( $\beta)(i) ;$ Fig. S2( $\beta)(i)$ ). The Hirshfeld plot features (Fig. 3(a)) clearly reflect the approach of the protonating hydrogen atom toward the cleft between the phenolic oxygen atom $(\mathrm{O}(7)$ in the CIF) and the adjacent $o$-nitro oxygen atom $(\mathrm{O}(1)$ (Table S6( $\beta)(\mathrm{i}))$, closer to the former. To either side the pyridinium $o$-hydrogen $\mathrm{H}(3)$ also approaches O(7) (evident on the cation Hirshfeld surface, Fig. 3a)) while a symmetry-related picrate hydrogen $\mathrm{H}(1)$ also approaches $\mathrm{O}(1)$. At the periphery of the column generated by the asymmetric unit, the approach between screw related p-nitro-oxygen and $\mathrm{CH}$-hydrogen atoms 
of anion and cation is evident. All of these features are reflected in the fingerprint plot (Fig. 5(a)), which, like nearly all others, is dominated by protonating hydrogen/phenoxide features. There is also clear evidence of $\pi . . . \pi$ interactions. Of further interest is the environment of the other $o$-nitro group oxygen atoms proximal to $\mathrm{O}(7)(\mathrm{O}(5,6))$ in the CIF, which, by virtue of the nitro-group being significantly twisted out of plane $\left(\mathrm{C}_{6} / \mathrm{CNO}_{2}\right.$ interplanar dihedral $\left.37.00(12)^{\circ}\right)$, are disposed with close contacts to a number of other atoms in an electronegative region of the anion which lies above it. This is evident in the lower central marker of the fingerprint plot.

The unit cell projections of the triclinic form (PYRPIC03) show the presence of inversion-related asymmetric units with approaches within the anion-cation pair very similar to those of the monoclinic form (Table 4), and lying obliquely, albeit quasi-normal, to $c$ in projection down that axis and stacking up it. These stacks are linked into sheets, which lie parallel to (110), by inversion-related cation-H...o-nitro-O approaches; the sheets are linked by similar interactions. Both anion...anion (inversion related; interplanar distance $3.48 \AA$ ) and anion...cation overlaps are found (Table S6( $\beta)($ ii); Figs. S1( $\beta)(i i)$, S2( $\beta)(i i))$. The former are more significant, the latter (and other) interactions more or less peripheral. The phenolic oxygen atoms closely approach the phenolate/o-nitro regions of inversion-related anions, readily seen in the $c$-axis projection. Although showing the same types of features in principle, the fingerprint plots of the two polymorphs differ significantly in respect of their $\pi \ldots \pi$ interactions and C-H...O hydrogen bonds (both with the donor and the acceptor groups being within the picrate ion) which are much more pronounced in the triclinic form, indicating closer and more numerous interactions of that type in that form. 


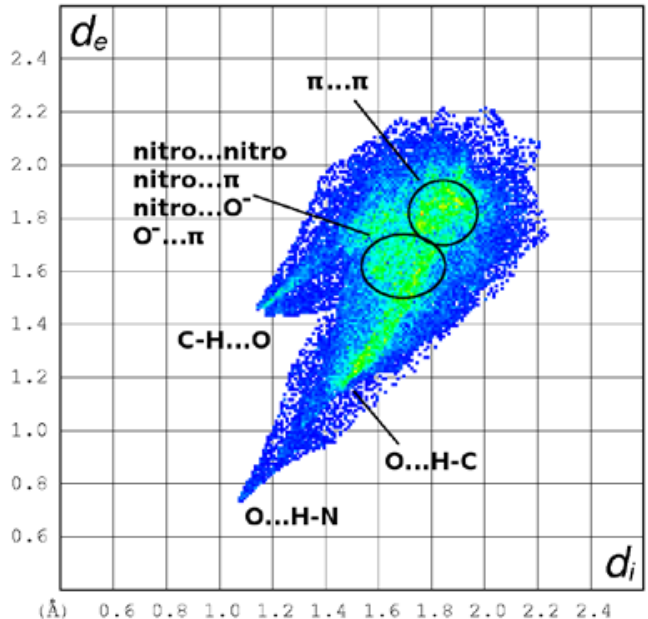

a) ( $\beta$ )(i) (pyH)(pic) (monoclinic)

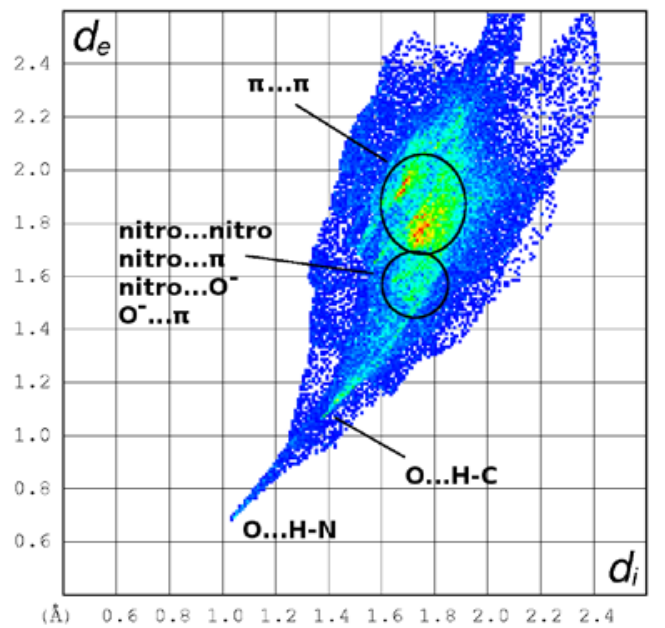

c) ( $\beta)($ iii) (pyH)(pic)(·naph)

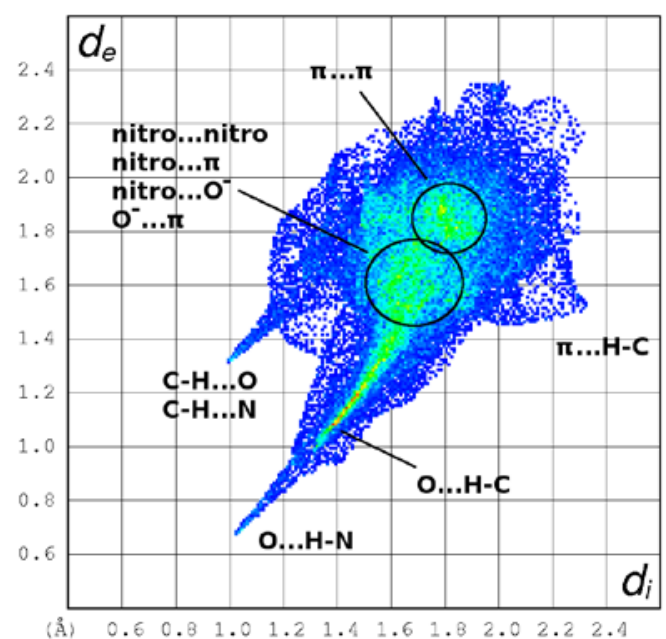

e) ( $\beta$ )(iv) (2mpH)(pic), molecule 2

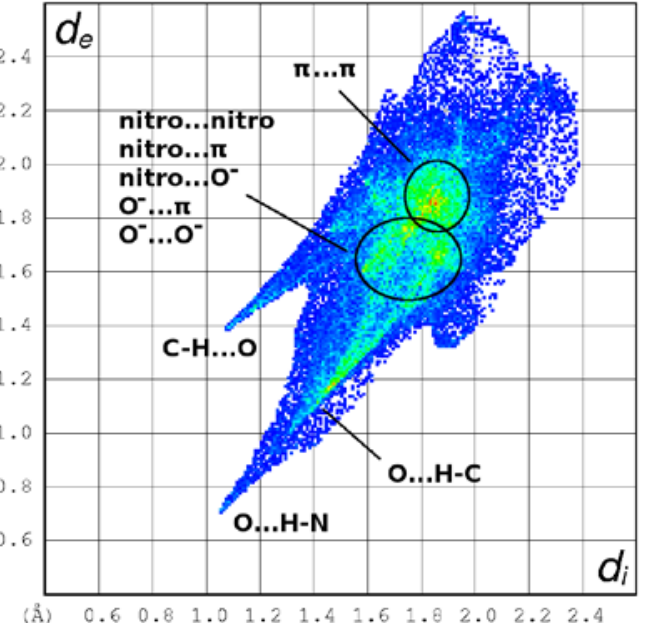

b) ( $\beta$ )(ii) (pyH)(pic) (triclinic)

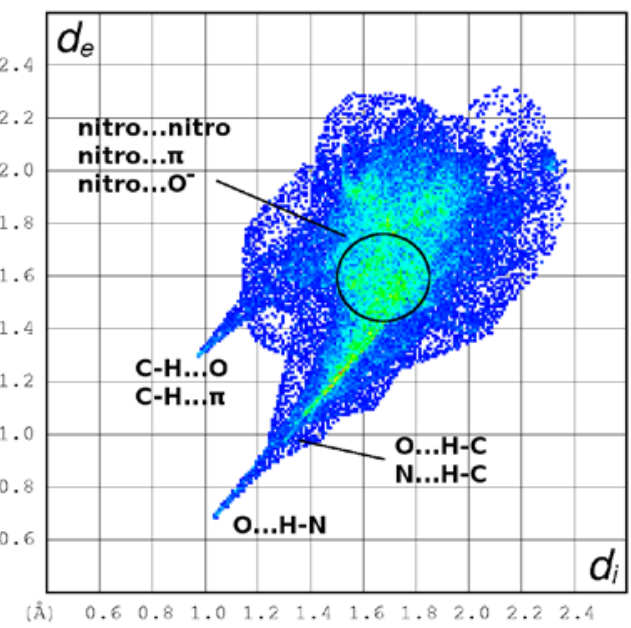

d) ( $\beta$ )(iv) (2mpH)(pic), molecule 1

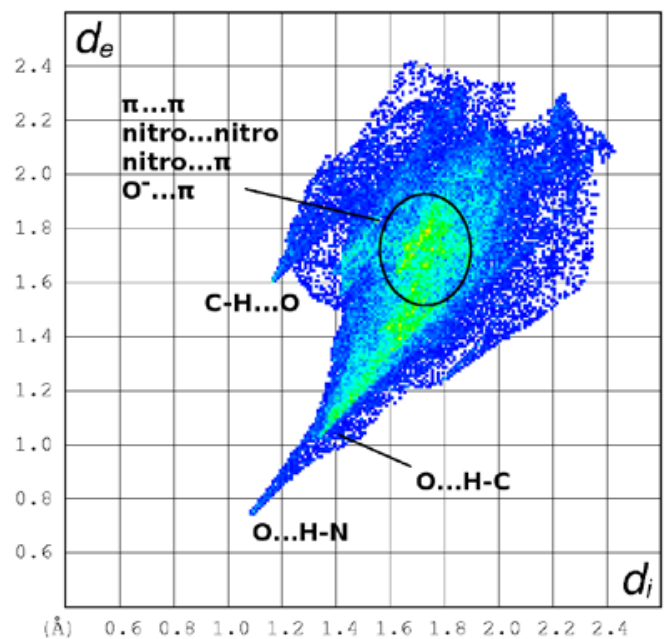

f) $(\beta)(v)(\mathbf{d p a H})(\mathbf{p i c})$

Fig. 5 a)-f) Fingerprint plots of group ( $\beta$ ) compounds: (substituted-) pyridinium salts. 
( $\beta$ ) (iii) Pyridinium picrate : 1-naphthylamine. The pyridinium-picrate interaction persists in the interesting ('Kofler's') ternary complex 'solvate' with 1-naphthylamine (PYNPCR ${ }^{20}$ ). Here, the latter unprotonated component does interact with the substrate via some degree of hydrogen-bonding by way of the $\mathrm{NH}_{2}$ group, but this appears to be relatively feeble as this component is disordered and interleaves the structure in a disordered fashion (Fig. S1( $\beta$ )(iii)), a serious impediment to any proper development of relevant Hirshfeld surface and fingerprint plots. One formula unit, again inclusive of an anion-cation pair, comprises the asymmetric unit of the structure; here, the two components lie oblique to each other (interplanar dihedral angle: $\left.67.11(3)^{\circ}\right)$ so that any $\mathrm{CH} . . . o-n i t r o-O$ support is absent (Fig. 2a)), perhaps accounting for the fact that phenoxy-O...N-py is slightly longer here than in the previous two phases, despite the oblique approach (Fig. S3( $\beta$ )(iii)). Although all components of the asymmetric unit, or their symmetry-related images, are parallel (Fig. S1( $\beta)(i i i)$ ), the predominant overlap is between pairs of inversion-related anions (interplanar distance $3.43 \AA$ ) (Fig. S2( $\beta$ )(iii)) with only peripheral interactions between the remainder. The unit cell projections of Fig. S1( $\beta$ )(iii) are unremarkable, although the projection down $a$ suggests an unrealistically close association of the cations about the centre of the cell. However, the projections down $(011,01 \overline{1})$ are very different, showing two sets of components to stack as parallel planes, oblique to these lines and forming sheets extended in these directions; the anion and cation components of the asymmetric unit are disposed in each sheet, with their hydrogen bonds linking them together. Notably, this is the only compound among all salts of Table 1 in which the C-H groups of the picrate moiety do not act as hydrogen bond donors, explaining the absence of the left spike usually found in the fingerprint plot (Fig. 5(c)). However, the remaining features of the fingerprint plot show a close similarity to those of the triclinic form of the unsolvated pyridinium picrate. The absence of diffuse features (e.g. as seen in (bpyH)(pic)( $\mathbf{M e C N})$ (Fig. 
8 b)), confirms that interactions between 1-naphthylamine and the picrate anion are negligible.

( $\beta$ ) (iv) 2-Methylpyridinium picrate. Extension of the pyridine ring system laterally, initially trivially by addition of a 2-methyl group, results in 2-methylpyridinium picrate, (2mpH)(pic). This compound, of the 'next simplest' aromatic base, 2mp, also the subject of study by others at room temperature (PEMCUG), ${ }^{37}$ crystallizes with twice the stoichiometric entity in the asymmetric unit, (Fig. 2a)) in the triclinic space group $P \overline{1}$. In both forms of the parent pyridinium salt, the planes of the cation and anion are quasi-parallel/coincident in each case (see above). Such is not the case here; in both ion-pairs of the asymmetric unit, the pair of planes are oblique to each other (Fig. 2a)), presumably a concomitant of the added hindrance imposed by the 2-methyl group. However, centrosymmetric overlaps are found between pairs of each of the independent anions although the interplanar spacings are larger than the previous $(3.54,3.33 \AA$ ) (Fig. S2( $\beta)(i v)$ ), with the closest contacts arising from the oxygen atoms of one of the $o$-nitro groups of each which are twisted well out of plane. As with the pyridinium salts, the transition to planar aza-aromatic base form raises additional possibilities for stacking interactions in the lattice, with differences in basicity of the nitrogen atom also expected to influence hydrogen-bonding interactions. A view projected down $c$ (Fig. $\mathrm{S1}(\beta)(\mathrm{iv})$ ) shows a segregation of the two types of aromatic units into sheets parallel to (010) stacking with parallel to near-orthogonal planes; projection down $a$ shows segregation of the two pairs into sheets normal to $c$, linked only by feeble (Ar)CH...o-nitro-O approaches. In fact, dissected thus and viewed again in projection down $c$ (Fig. S1( $\beta$ )(iv)) it is seen that the basic motif may be considered to be a two-layered ribbon for each component of the asymmetric unit, the two components displaced by 0.25 in $x$. 
Within each component of the asymmetric unit, (Fig. 2(a)), we see that the proton formally bound to the nitrogen atom is 'chelated' by picrate phenoxide- and nitro-oxygen atoms in the usual way, being 'three-coordinate', with supportive $\mathrm{CH}$...o-nitro-O approaches (the former now from the methyl $\mathrm{C}$ group), which persist despite the oblique approach of the aromatic planes (Fig. 2(a)). Notable among the 'peripheral' interactions are those arising from oxygen atoms in $o$-nitro groups twisted out-of-plane and approaching $\eta^{\mathrm{n}}$ to nearby cation aromatic rings $(\mathrm{O}(\mathrm{n} 61) \ldots \mathrm{C}(0 \mathrm{~nm}) \sim 3.4 \AA$ (Table $\mathrm{S} 6(\beta)(\mathrm{iv}))$. All these features are clearly evident in the fingerprint plots (Figs. 5 d), e)). The two fingerprint plots of the independent picrate anions in the asymmetric unit are very similar, much more so than in the fingerprint plots of the two polymorphic species of pyridinium picrate, but confirm that there is only a pseudo-symmetric and not a truly crystallographically symmetric relationship between them by the way the (faint) $\pi$-interactions feature. The anion of component 1 can be identified as the acceptor and the anion of component 2 as the donor of a C-H... $\pi$ interaction, whereas only anion 2 possesses an upper central marker indicative of faint $\pi . . . \pi$ interactions. Moreover, anion 1 is the acceptor and anion 2 the donor of a C-H...N hydrogen bond of a type that rarely occurs in the array of compounds investigated in this study.

( $\beta$ ) (v) (2-Pyridinium)(2-pyridyl)amine picrate. The parent 'dipyridylamine', 'dpa', has been studied in its neutral, protonated and various coordinated forms according to circumstances; the parent base is found in three different polymorphs, ${ }^{47}$ in all of which the molecules are of an anti-disposition, with one of the pyridyl nitrogen atoms lying 'cis' to the central NH group, so as to hydrogen-bond with a similar N-C-C-NH array which may be symmetry/centrosymmetrically related, and the other pyridyl nitrogen atom directed away. However, in the monoprotonated form, which has been the subject of a recent extensive study ${ }^{15}$ in diverse salts, as well as in the present study, we find the monocation in an essentially planar form, 
with the pair of pyridyl nitrogen atoms 'endo' and bridged by the added proton which lies closer to $\mathrm{N}(22)$ than $\mathrm{N}(12)(\mathrm{N}(12) \ldots \mathrm{N}, \mathrm{H}(22) 2.616(2)$, 1.83(2) $\AA$ ), and consistent with the basicity (in the solid state) being greater for the pyridyl- rather than the anilino- $N$ centre; a single formula unit comprises the asymmetric unit of the present structure. As is apparent from a view of the lattice down $a$ (Fig. $\mathrm{S} 1((\beta)(\mathrm{v}))$, the aromatic entities, picrate and (pyridinium)(pyridyl)amine, this time do not lie in parallel planes (the cation...anion interplanar dihedral angle is $\left.22.81(14)^{\circ}\right)$, although there are extensively overlapping inversion-related anion pairs, cations less extensively so, and a close anion : cation overlap (Fig. S2( $\beta)(v)$, also evident in projection down $b($ Fig. $\mathrm{S} 1(\beta)(\mathrm{v}))$, as well as inclined anion : cation pairs where significant overlap in projection occurs (Table S6( $\beta)(v)$ ). Hydrogen bonding involves both $\mathrm{NH}$ centres, the anilino hydrogen atom being chelated by a quasinormal picrate anion approach between phenoxide- and o-nitro-O atoms $(\mathrm{N}, \mathrm{H}(1) \ldots \mathrm{O}(1 ; 21)$ 2.758(2), 1.96(3); 3.121(3), 2.44(3) $\AA$ ) as seen in the HS display (Figs. S3( $\beta)(v)$ ), while the endo-chelated pyridinium/protonic hydrogen is contacted by a single nitro-O. In the fingerprint plot, Fig. 5(f), the central marker indicative of $\pi \ldots \pi$ interactions and the marker for interactions involving the nitro group have merged, which is in turn an indication for the preponderance of $\mathrm{C} . . . \mathrm{C} / \mathrm{N}$ contacts in the $\pi \ldots \pi$ interaction being shorter than usual, with the nitro interactions being less significant than in other compounds. The right spike representing the $\mathrm{N}-\mathrm{H} . . . \mathrm{O}$ ion-pair hydrogen bond is as dominant as in most compounds, but the C-H...O hydrogen bonds (left spike, with the picrate $\mathrm{C}-\mathrm{H}$ groups being the donors) are quite distant and less important in the crystal packing.

Pyridinium-picrate interactions: theoretical calculations. The fundamental ion-pair interactions have been further explored theoretically for a gas-phase picrate-pyridinium ion pair, adopting that of PYRPIC02 ${ }^{11 \mathrm{c}}$ as a starting configuration; an isolated picrate anion has 
also been the subject of study ( $c f$. Table 3). The geometries of the isolated ion pair have been optimized freely, allowing maximization of the total intermolecular interaction energy between the ions, employing diffuse functions to cover the long-range interactions sufficiently. The results are interesting: For the individual species the values for the resulting internal non-hydrogen bond lengths (theoretical) all lie between those for the isolated picrate and picric acid species; minor exceptions to the generalization are found among the angles, among the pendants, and exocylic to the aromatic ring of the picrate (Table 3). Upon geometry optimisation of the contact pair, the protonic hydrogen remains associated with the base, as one might expect, but with a rather more linear approach than in the experimental geometries of many of the other pairs of interest: H...phenolic-O, pyridine- $\mathrm{N}$ are 1.490, 1.081 $\AA$, with N-H...O $156.7^{\circ}$. The pyridinium-H...o-nitro-O distance is $2.357 \AA$ and the pyridinium ortho-H...phenolic-O $2.761 \AA$, so that the latter has not displaced the former 'chelating' mode of the protonic hydrogen in importance in this case. The combination of these interactions has the effect of creasing the picrate aromatic plane (still with considerable quinonoid character) across the line through the pair of carbon atoms ortho to the phenolic grouping, and, concomitantly, although the pair of $o$-nitro $\mathrm{CNO}_{2}$ planes are twisted relative to the aromatic plane, they are now mirror-related to the plane normal to the ring and through the phenolic $\mathrm{CO}$ and the para $\mathrm{CN}$ lines. In the picrate group, atomic charges are generally similar and inbetween those of the isolated picric acid and picrate species (Table 3 (footnote)), except in the vicinity of the phenolic CO group and the neighbouring $o$-nitro oxygen atoms; in particular, the charge on the phenolic oxygen atom is appreciably more negative.

\section{$(\gamma)$ Aza-alicyclic base salts - saturated derivatives of pyridinium picrate}

( $\gamma$ ) (i) Piperidinium picrate. Before embarking on consideration of the salts of more extended aza-aromatic bases, as available in the literature as summarized in Table 3, we were 
interested to consider the forms associated with the non-aromatic non-planar cyclic counterparts, as expressed in piperidine and morpholine, entailing tetrahedral nitrogen atoms each with two associated hydrogen atoms, and in which acid-base interactions, entailing these, and aromatic interactions arising from the anions only, might be expected (which is not to overlook the importance of anion stacking in other arrays). Indeed, in all of these, we find anion stacks, interspersed in diverse ways with cation/solvent arrays, to be a fundamental determinant in the organisation of the structures. During the present work, the structure of piperidinium picrate, (pipH)(pic) (VAZJAI), ${ }^{21}$ together with that of morpholinium picrate (morH)(pic) (KOMTUC) (two sources ${ }^{5}$ (see below)) (Figs. S1( $\left.\left.\gamma\right)(i, i i i)\right)$ were reported. In

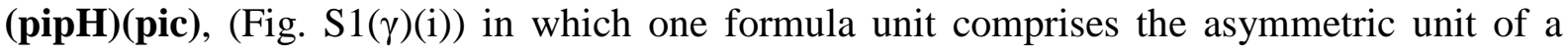
triclinic cell, one of the $\mathrm{NH}_{2}$ hydrogen atoms interacts with phenolic and adjacent/'chelating' $o$-nitro oxygen atoms in the manner found in the pyridinium salt (supported by a rather distant CH...O approach (Scheme 4)), the other with the other oxygen atom of a centrosymmetrically related o-nitro group; the 'plane' of the cation here lies oblique to those of the parallel anion aromatic planes. A considerable overlap, evident in the cell projection down $a$ of Fig. S1( $\gamma)(\mathrm{i})$ as well as Fig. S2( $\gamma)(\mathrm{i})$, is found between the centrosymmetrically related anions (interplanar distance $3.45 \AA$ ). Both dominating types of interactions - the cation-anion N-H...O hydrogen bond and the anion-anion aromatic overlap - are very pronounced in the fingerprint plot (Fig. 6a)) for this compound. Moreover, C-H...O hydrogen bonds and nitro-guided interactions are clearly discernible on the fingerprint plot, all of this in contrast to the fingerprint features of the monopiperidine solvate of the same compound presented in the following section. 


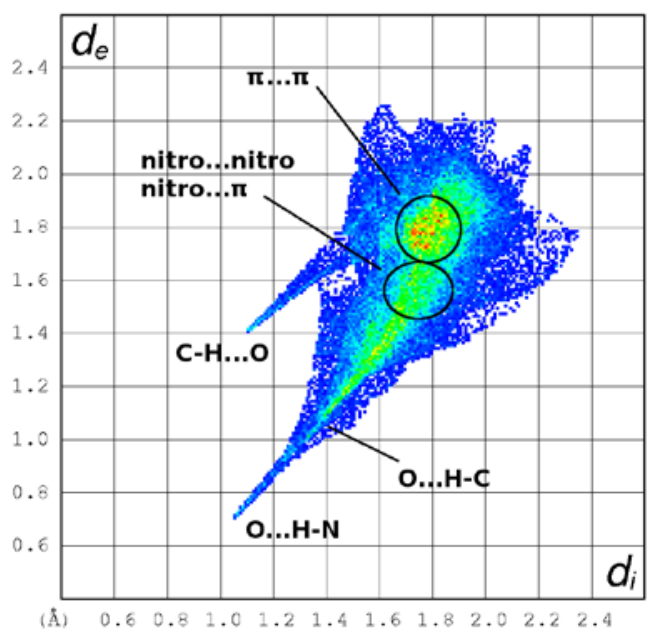

a) $(\gamma)(\mathrm{i})(\mathbf{p i p H})(\mathbf{p i c})$

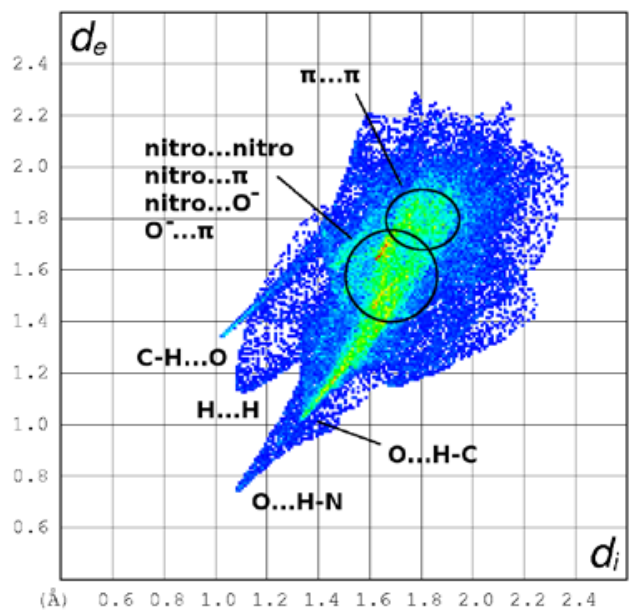

c) $(\gamma)(\mathrm{iii})$ (morH)(pic), molecule 1

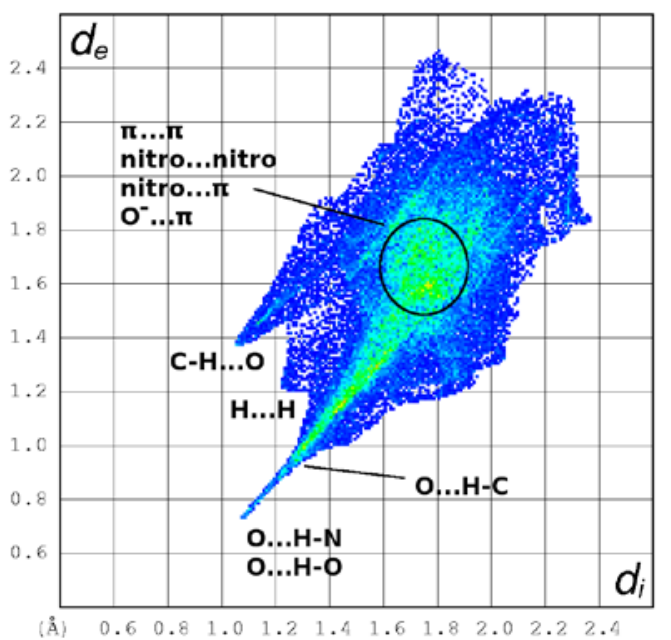

e) $(\gamma)(\mathrm{iv})(\mathbf{m o r H})(\mathbf{p i c})(\mathbf{x} 2)\left(\cdot \mathbf{H}_{2} \mathbf{O}\right)$, mol. 1

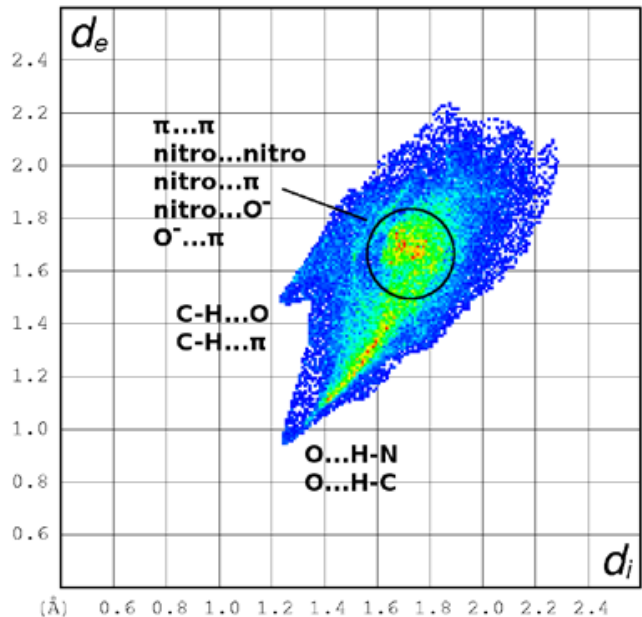

b) $(\gamma)(\mathrm{ii})(\mathbf{p i p H})(\mathbf{p i c})(\cdot \mathbf{p i p})$

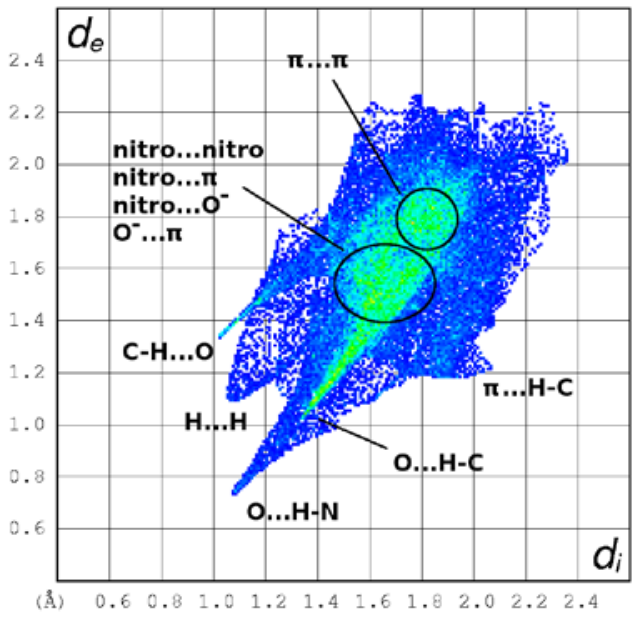

d) $(\gamma)($ iii) (morH)(pic), molecule 2

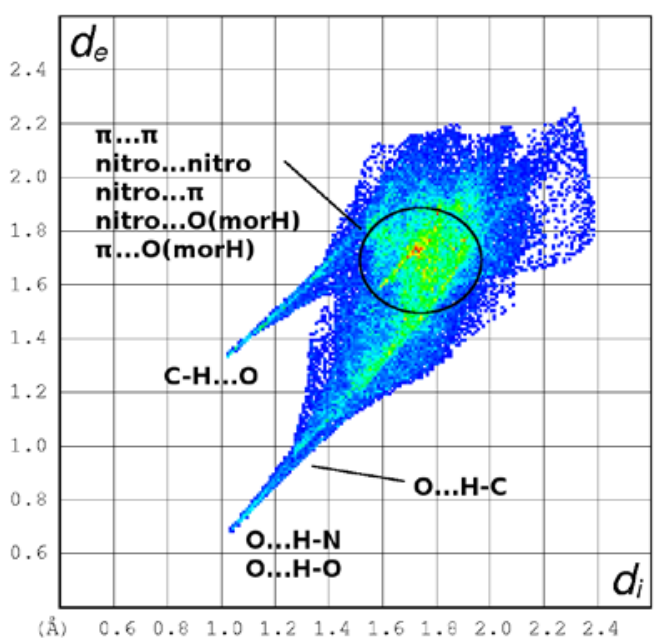

f) $(\gamma)(\mathrm{iv})(\mathbf{m o r H})(\mathbf{p i c})(\mathbf{x} 2)\left(\cdot \mathbf{H}_{2} \mathbf{O}\right)$, mol. 2

Fig. 6 a)-f) Fingerprint plots of group $(\gamma)$ compounds: saturated derivatives of pyridinium picrate. 
( $\gamma$ ) (ii) Piperidinium picrate monopiperidine solvate. Previously (pipH)(pic) was reported as prepared by evaporation of an ethanol solution of equimolar picric acid and piperidine ${ }^{21}$; our own crystallization from neat piperidine yielded a different product: piperidinium picrate monopiperidine solvate, '(pipH)(pic)(·pip)'. This compound crystallizes with one formula unit, devoid of crystallographic symmetry, comprising the asymmetric unit of the structure in a monoclinic $P 2_{1} / n$ cell. The protonated piperidine unit (labelled 2) has a hydrogen-bonding association with the picrate phenoxide-oxygen atom via one of the $\mathrm{NH}_{2}$ hydrogen atoms, more distant than in all of the other cases that exhibit this fundamental kind of anion-cation N-H...O hydrogen bond (N(21), H(21A)...O(1) 2.814(2), 2.25(2) (Fig. 2 c)). The other hydrogen atom is hydrogen-bonded to the unprotonated piperidine unit (labelled 1) with a remarkably short N-H...N hydrogen bond (N(21), H(21B)...N(11) 2.690(2), 1.81(2) $\AA$ ), the shortest in all compounds presented here. This unique motif suggests that the array may be regarded as comprised of (pipHpip)(pic) molecular units or clusters (Fig. 2 c)), although formally a piperidine monosolvate of the salt (pipH)(pic). However, in the (pipHpip) ${ }^{+}$unit, (see below), the protonated piperidine (component 2) remains clearly defined as such, by virtue of the asymmetries found in the associations of the relevant nearby hydrogen atoms. Nonetheless, the interactions between the two base hydrogen atoms $H(11,21 a)$ and the picrate anion $\mathrm{O}(1,15)$ and $\mathrm{O}(1,25)$ 'chelates' are quasi-symmetrical, as if between bidentate and tridentate components (Scheme 4). Supporting peripheral CH...o-nitro-O approaches are also evident. The fingerprint plot of the picrate entity is quite different to most of the other picrate fingerprint plots in Fig. 6 b) (in particular, that of the non-solvated piperidinium picrate) in that the $\mathrm{N}-\mathrm{H} . . . \mathrm{O}$ (phenoxy) interaction is not dominant, but as distant as the $\mathrm{CH}$...o-nitro-O contacts, in accordance with the features imposed by the unique (pipHpip)(pic) array 
discussed above. Also, C-H...O hydrogen bonds originating from the picrate anion as donor are nearly absent, being supplanted by C-H... $\pi$ interactions.

The relative distances of the interactions suggest some comparability of piperidine and picrate base strengths in the solid state. The geometries of the protonated and unprotonated non-hydrogen skeletons are closely similar (Table S1), the piperidine skeletal geometry being much less susceptible to variation upon protonation than is the case in the aromatic counterparts (see below). Counterpart C-C distances differ by less than $0.00_{4} \AA$ between the two species (pip, $\mathrm{pipH}^{+}$) for similar bonds, and C-N by ca $0.01_{3} \AA$; the angles at the nitrogen atoms are 111.65(9) (pipH) and 112.75(9) ${ }^{\circ}$ (pip), with other variations $<1.3^{\circ}$. The differences in counterpart torsion angles are $<1.8^{\circ}$.

The fingerprint plot suggests that $\pi . . . \pi$ interactions are extraordinarily important in this modification - even more significant than in the unsolvated form where the intense central marker is quite strong already - because the central markers for aromatic overlaps and nitrointeractions have merged below contact distances of 3.6 . As in the unsolvated structure, the

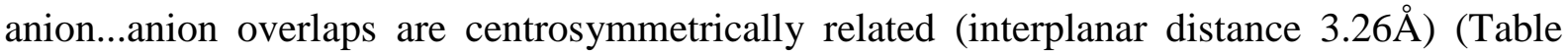
S6( $\gamma)($ ii); Fig. S2( $\gamma($ (ii)), and visualized in the Hirshfeld-surface projections of Figs. 7a) and b). Six pronounced red markers on both sides of the surface show that all six individual C-C contacts to the neighbouring picrate ions in the asymmetric units below and above are shorter than the corresponding sum of the carbon van-der-Waals radii. This is a most unusual situation, certainly without parallel in the present array of investigated compounds, $\pi-\pi$ interactions usually only giving rise to a web-like pattern on the Hirshfeld surface ( $c f .$, e.g., Fig. 3a), same colour scale).

More extensively, the lattice array, viewed down $a$, is an elegant honeycomb (Figs. 7c),d), S2( $\gamma($ ii)), with four-sided cavities, the almost exactly equi-dimensional sides (in an almost equi-dimensional cell) built up from hydrogen-bonded (pipHpip) ${ }^{+}$units to enclose 
infinite slipped stacks of picrate anions. These inversion/translation generated columns involve picrate units alternating in orientation as antiparallel dipoles, spacing $\sim a / 2(a=$ $6.5862(5) \AA$ (see above)), with projections perpendicular to the aromatic ring plane showing almost exact overlap on each anion by its neighbours to either side (Figs. 7c), S2( $\gamma$ )(ii), also evident on the Hirshfeld surface in Figs. 7a) and b), as discussed above), various inter-atom contacts (C...C, N...O) being less than 3.2 - $3.3 \AA$. The four-sided cavities apparent in projection down $a$ can be considered to be the result of the intersection of orthogonal sheets of the (pipHpip) ${ }^{+}$units, the intersection channels comprising lipophilic entities lined by methylene group hydrogen atoms on a pair of opposed sides, and with protonated polar portions oriented towards the enclosed anion stacks on the other pair. The void representation (Fig. 7d)) shows how the infinite column of stacked picrate ions occupies the centre of the unit cell without any space between the ions. In contrast, there are significant voids surrounding the cations and solvent molecules, allowing only directed hydrogen-bonded contacts.

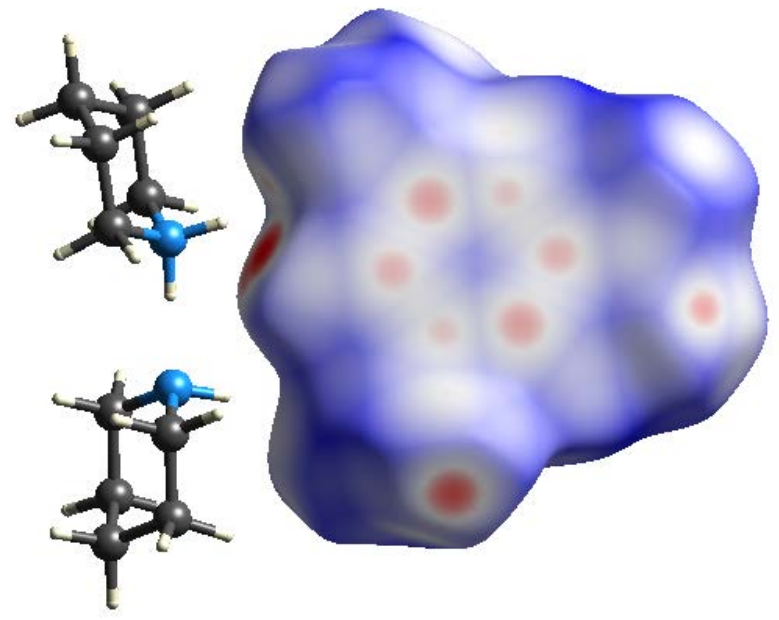

a)

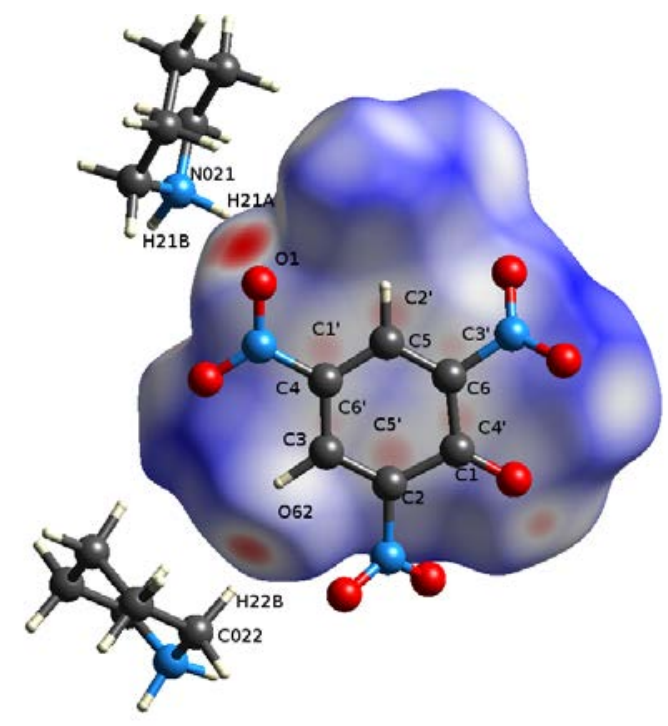

b) 


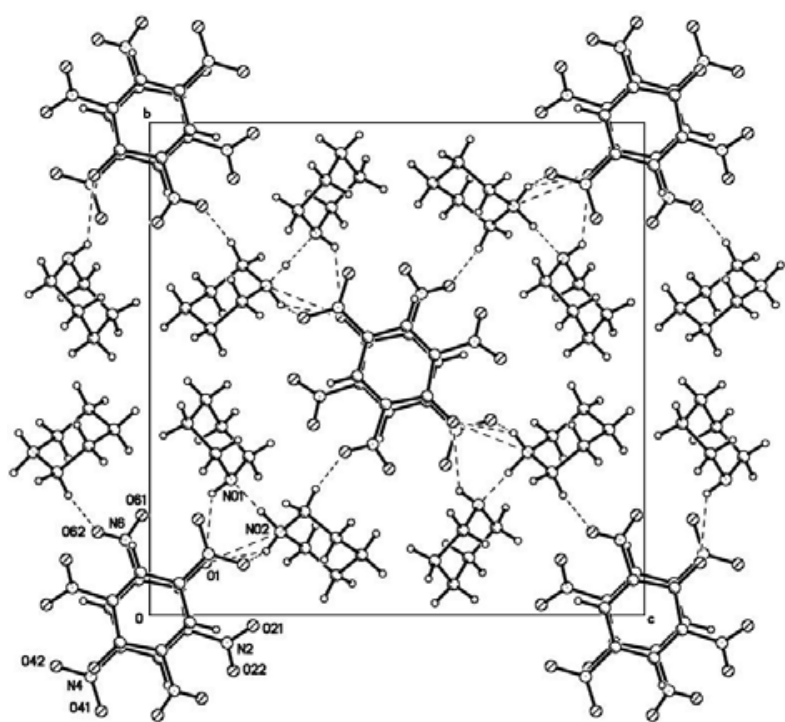

c)

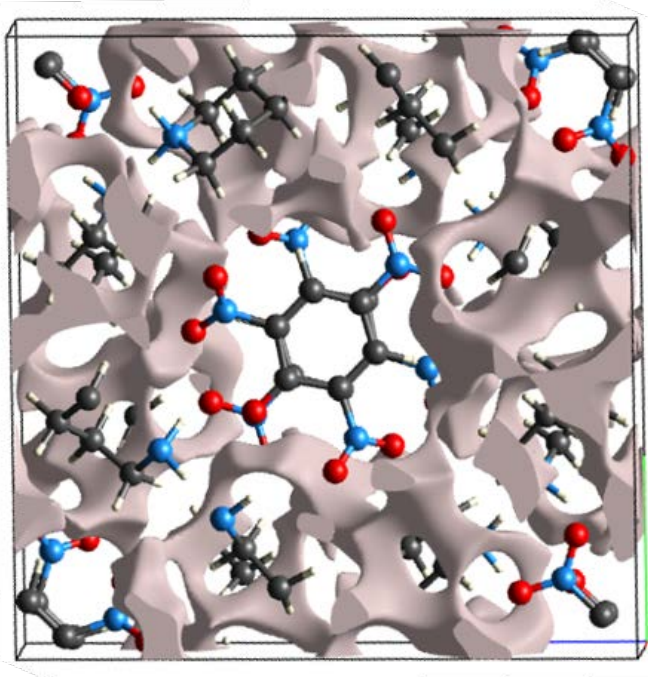

d)

Fig. 7 a)-b) Hirshfeld surface, c) unit cell projection and d) associated void representation (electron-density isovalue $=0.002$ a.u $)$ of $(\gamma)(\mathrm{ii})(\mathbf{p i p H})(\mathbf{p i c})(\cdot \mathbf{p i p})$, all projections down $a$.

( $\gamma$ ) (iii) Morpholinium picrate. The structure of morpholinium picrate (morH)(pic) has also been recorded in two determinations (ref. ${ }^{22}$ (KOMTUC) describing the more precise). Two formula units comprise the asymmetric unit of the structure in space group $P \overline{1}$. Replacement of one methylene unit of piperidine by oxygen to give morpholine would be expected to affect the lipophilicity of the molecule, as well as its polarity, and it is certainly associated with some quite substantial changes in the nature of the lattice of the picrate salt. The aggregation of the morpholinium and picrate entities may be considered in the first instance to be 'molecular'/ion-pair, with one of the $\mathrm{NH}_{2}$ hydrogen atoms of each cation interacting with a phenol-O/o-nitro-O 'chelate' and distant supporting $\mathrm{CH} . . \mathrm{O}$ approach (Scheme 4). In this complex, $c f$. the previous, the other $\mathrm{NH}_{2}$ hydrogen atom interacts with the morpholinium oxygen atom of the other ion-pair. Both independent picrate ions of the asymmetric unit experience the same pattern of intermolecular interactions, this being the only compound of this study which displays all possible interaction types: the fundamental cation-anion N-H...O hydrogen bond, additional C-H...O, C-H... $\pi$ interactions with the picrate aromatic system 
being the acceptor, and close $\mathrm{H} . . . \mathrm{H}$ contacts giving rise to a strong middle spike in the fingerprint plots. Regarding interactions not involving hydrogen atoms, the usual close nitroguided interactions are here accompanied by close phenoxy-O... $\pi$ and phenoxy-O...nitro interactions (O...O and $\mathrm{O} \ldots \mathrm{N}$ as close as $3.0 \AA$ (Table S6( $\gamma)(\mathrm{iii})$ ), together with $\pi \ldots \pi$ interactions that are overall more distant than in the previous piperidinium compounds. Anion overlaps here are inversion related (Table S6( $\gamma)($ iii); Fig. S2( $\gamma)($ iii)) with interplanar spacings of 3.45, 3.46 $\AA$. Sheets of cations are disposed about the plane $y=0$, anions about $y=1 / 2$ (Fig. S1( $\gamma)(\mathrm{iii}))$

( $\gamma$ ) (iv) Morpholinium picrate hemihydrate. In the present work, crystallization of picric acid from 'neat' morpholine has yielded: morpholinium picrate hemihydrate, $2[(\mathbf{m o r H})(\mathbf{p i c})]\left(\cdot \mathbf{H}_{2} \mathbf{O}\right)$. This compound crystallizes in space group $C 2 / c$, with one such formula unit, i.e. two independent cations, two independent anions and one water molecule, comprising the asymmetric unit. The projection down $b$ shows the disposition of cations and anions in alternating sheets about $z=0,1 / 4$ (etc.); while the gross stacking is not dissimilar to that in the (pipHpip) ${ }^{+}$adduct, the anions in the anion stacks are now not evenly spaced, but disposed pairwise with a spacing of $3.41 \AA$ (Figs. S1( $\gamma)(i v)$ ). In detail, the structure is more complex and interesting, best appreciated by reference to Fig. 2d), which shows the asymmetric unit of the structure to comprise a motif built as a cluster around the pair of anions linked to either side by one cation and the water molecule, the second cation linking to the latter (in the first instance) rather than either of the phenoxide oxygen atoms. Anions 1 and 2 have their phenoxide oxygen atoms bridged from one side of their 'plane' by the two $\mathrm{NH}_{2}$ hydrogen atoms of $(\mathrm{morH})^{+}$cation 1 (N(011), H(011a)...O(11) 2.706(2), 1.88(3); N(011),

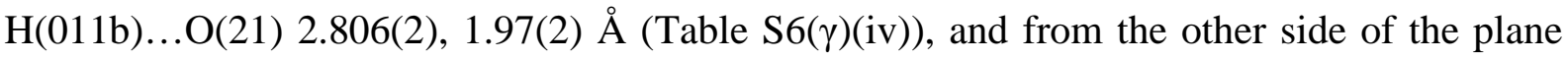
by the two hydrogen atoms of the water molecule in the manner identified by ref. ${ }^{8}$ as 'synthon 
II', one interacting directly with $\mathrm{O}(11)(\mathrm{O}(01 \mathrm{w}), \mathrm{H}(0 \mathrm{wa}) \ldots \mathrm{O}(11) 2.761(2), 1.81(3) \AA)$, the other being disposed between $\mathrm{O}(21)$ and the adjacent o-nitro oxygen $\mathrm{O}(261)(\mathrm{O}(1)$, $\mathrm{H}(0 \mathrm{wb}) \ldots \mathrm{O}(21)$; $\mathrm{O}(261) 3.019(2), 2.24(4)$; 2.938(2), 2.12(4) $\AA$ ). This may be visualised in the Hirshfeld surface representation (Fig. 3e)), with the water molecule/cation comprising a composite base. The $\mathrm{NH}_{2}$ hydrogen $\mathrm{H}(021 \mathrm{a})$ of cation $2(1 / 2-x, 1 / 2-y, 1-z)$ also interacts with $\mathrm{O}(11,161)$, so that paired systems stack above each other, with cations 2 interleaving and bridging them by the associated pair of $\mathrm{NH}_{2}$ hydrogen bonds, one directed toward the inversion-related water molecule oxygen of the other pair as described, and one directed towards picrate anion 2, contacting the phenoxide $(\mathrm{N}(021), \mathrm{H}(021 \mathrm{~b}) \ldots \mathrm{O}(221) 2.964(2)$, 2.34(3) Å), with, also, a further close contact to a p-nitro oxygen atom of an anion in an adjacent stack (N(021), $\mathrm{H}(021 b) \ldots \mathrm{O}(141)(x-1 / 2,1 / 2-y, z-1 / 2) 2.914(2), 2.53(2) \AA ̊)$, the water molecule being enclosed within the 'sandwich' as described. The stacking of the pairs, up $a$, is thus ...HHTTHHTT..., the result being that each anion stack presents a pair of faces, directed towards $\pm b$ with no hydrogenic interactions, confronting a similar face of the next stack, while the other pair of faces, directed $\pm c$, interact with cations and water molecules, with some species bridging to the next stack (Fig. S1( $\gamma)(\mathrm{iv})$ ). Interestingly, in this compound, $c f$. its anhydrous counterpart, the cation oxygen atoms do not appear to be involved in hydrogen approaches; instead we find, notably between anion 1 and cation $1(1-x, y, 1 / 2-z)$, $\mathrm{O}(014) . . . C(11-16)$ lying between 3.162(2)-3.419(2) $\AA$ showing a remarkable close ' $\eta^{6}$ approach of that morpholinium oxygen atom to the picrate aromatic ring. Morpholinium cation skeletal geometries are closely similar throughout both compounds, and, with due allowance for the differences arising from the presence of the oxygen atom, closely similar to those of the piperidinium species (above), all examples being in the usual 'chair' conformation (Table S1). The fingerprint plots of the two independent picrate anions in the asymmetric unit of this hydrate are remarkably different from each other (Figs. 6e),f)), and from those of the 
two independent picrates in the non-hydrated modification (Figs. 6c),d)). In detail, anion 1 possesses close dihydrogen interactions in contrast to anion 2, a less intense central marker being indicative of the absence of interactions involving the morpholine oxygen atom in the fingerprint plot of anion 1 which are present in that of anion 2, as well as a more diffuse left spike. However, in the fingerprint plots of both anions, the central regions for nitrointeractions and $\pi \ldots \pi$ interactions have merged as for the solvated piperidinium compounds. Comparing the piperidinium and morpholinium (aliphatic) systems (c) with the pyridinium (aromatic) systems (b), the most obvious difference is the significance of the $\pi$-stacking interactions between the anions in the former case, as expected given the absence of aromatic counterparts in the cations. Otherwise, in general, the interactions between the cation and the picrate oxygen triad (as compared in Scheme 4), guided by the strong N-H...O hydrogen bond, dominate the packing in all cases, with accompanying $\mathrm{C}-\mathrm{H} . . . \mathrm{O}$ and nitro...nitro/nitro... $\pi$ interactions being very similar between systems in $(\beta)$ and $(\gamma)$ as well.

\section{(ס) Bipyridinium picrates and derivative systems}

(ס) (i) 2,2'-Bipyridinium picrate. With extension of the size and denticity of the aza-aromatic base, the simpler of the structures of the monoprotonated picrates are found for 2,2'-bipyridine ('bpy'), the same with an aromatic fusion between the pair of pyridine rings in 1,10phenanthroline ('phen'), the same laterally extended as in 2,9-dimethyl-1,10-phenanthroline ('dmp'), and bpy extended with a further pyridine in 2,2':6',2"-terpyridine ('tpy'), or separated as a pair of pyridine rings linked by a notionally competitive amine group in bis(2-pyridyl)amine ('dpa'). Only the bpy species has been isolated as a solvate, and all crystallise with one formula unit as the asymmetric unit of the structure. 2,2'-bipyridine offers interesting motifs by virtue 
of the predominantly cis dispositions of the pairs of nitrogen atoms in its mono-protonated salts. Again, at the outset of the present project, a report of the structure of unsolvated 2,2'bipyridinium picrate (bpyH)(pic) crystallized from methanol was recorded (UCOFUO) ${ }^{23}$

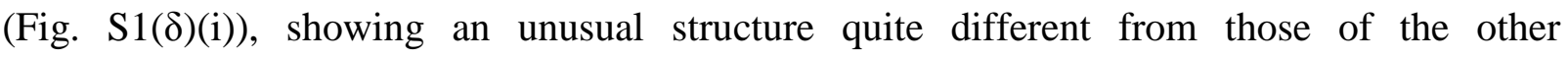
bipyridinium forms hereunder, but precursive of the arrangement found in (tpy)(pic) (below). Here we find that the p-nitro group rather than the phenolic oxygen atom of the picrate interacts with the protonated ring nitrogen atom of the 2,2'-bipyridinium in its cis form, together with the adjacent $\mathrm{C}-\mathrm{H}$ hydrogen. The O...O...O triad approaches $\mathrm{CH}-\mathrm{CH}$ pairs on the two bipyridinium rings resulting in more complex, more distant phenolic-oxygen...CH hydrogen interactions leading to the formation of hydrogen-bonded chains. The fingerprint plot (Fig. 8a)) confirms that the N-H...O hydrogen bond between cation and anion is not dominant (being as short as the C-H...O hydrogen bonds) and does not involve the phenoxy oxygen atom, clearly an exception in the array of compounds studied. C-H...O interactions with the donor group being located within the picrate anion (left spike) are nearly as pronounced as the hydrogen bonds to picrate-oxygen acceptors in the right spike, this being also an unusual phenomenon. Additionally, C-H... $\pi$ interactions between pairs of symmetry-related picrate anions are obvious from the fingerprint plot.

The reported crystal structure is nicely precise, at $120 \mathrm{~K}$, with the picrate geometry clearly emphasising the quinonoid contribution, and with the $\mathrm{C}-\mathrm{N}$ distance of the $p$-nitro group shorter than the corresponding values for the ortho counterparts. Substantial anion...cation and centrosymmetric anion pair overlaps (spacing $3.21 \AA$ ) are found (Fig.

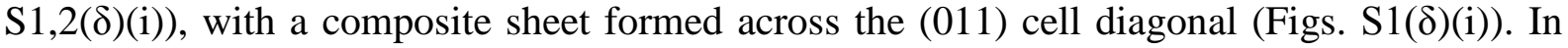
this compound, again, markers for the $\pi . . . \pi$ interactions merge which those of nitro...nitro, nitro... $\pi$, nitro...phenoxy, and phenoxy... $\pi$ interactions, all being present by way of various approaches (cf. Table S6). 


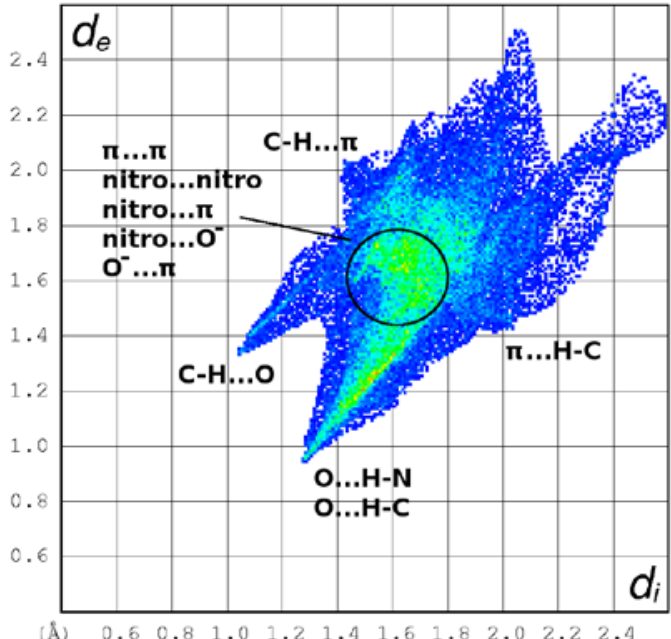

a) $(\delta)(\mathrm{i})$ (bpyH)(pic)

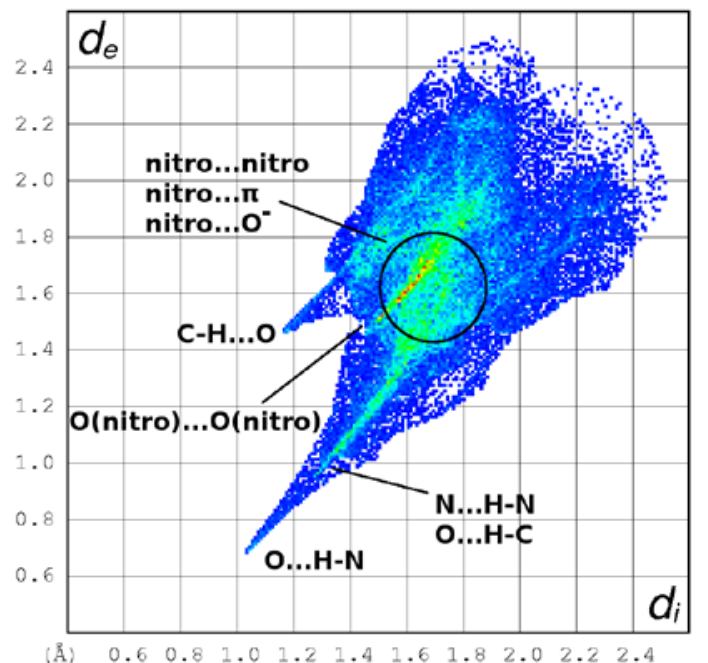

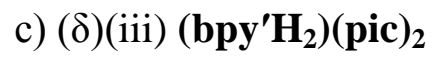

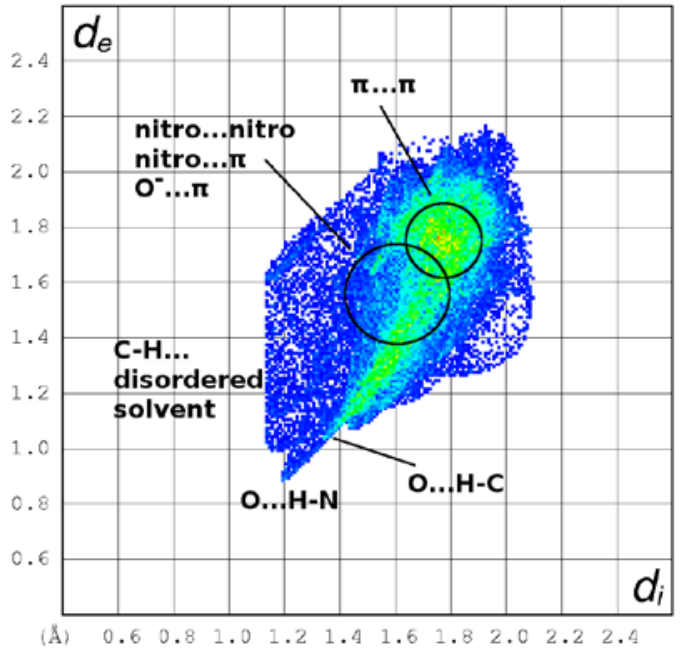

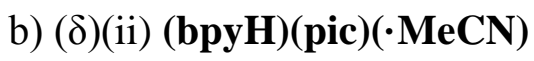

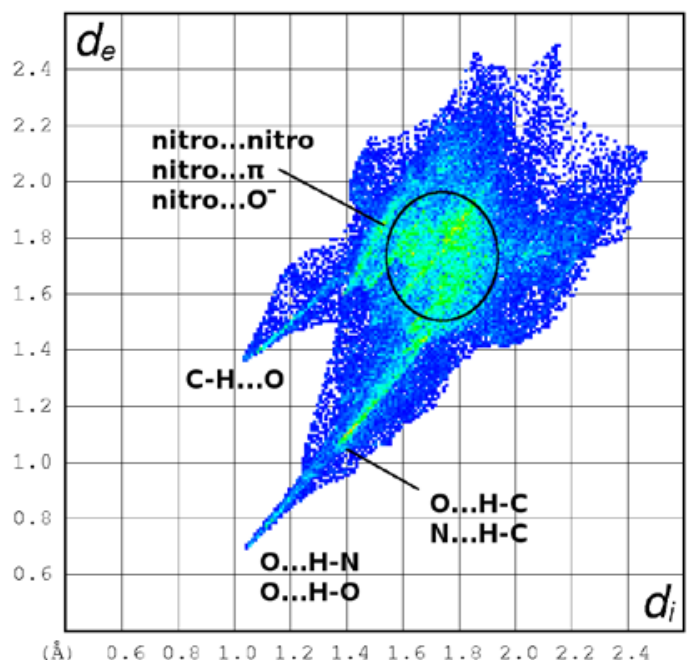

d) $(\delta)\left(\right.$ iv) $\left(\mathbf{b p y}^{\prime} \mathbf{H}_{2}\right)(\mathbf{p i c})_{\mathbf{2}}\left(\cdot \mathbf{H}_{\mathbf{2}} \mathbf{O}\right)$

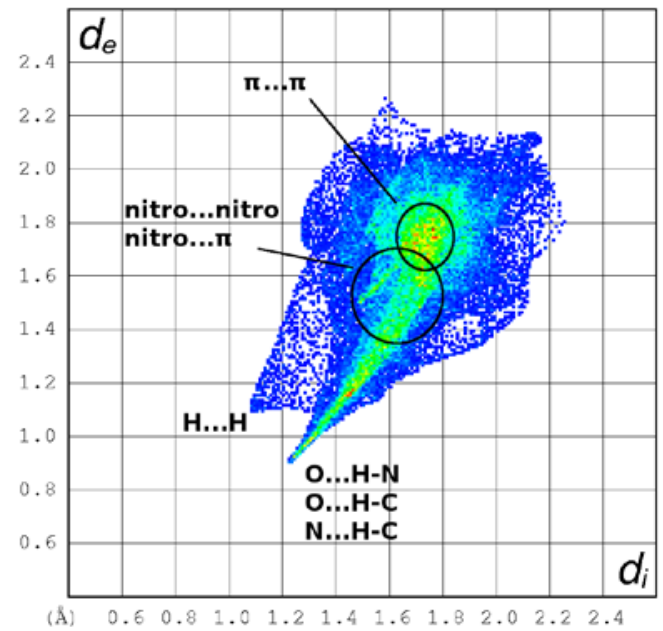

e) $(\delta)(\mathrm{v}) \mathbf{( t p y H ) ( p i c )}$

Fig. 8 a)-e) Fingerprint plots of group ( $\delta$ ) compounds: bi- and terpyridinium picrates. 
(ס) (ii) 2,2'-Bipyridinium picrate mono-acetonitrile solvate. In our own synthesis, which employed acetonitrile as solvent, a different (solvated) form was obtained: $\mathbf{2 , 2}$ '-bipyridinium picrate mono-acetonitrile solvate, (bpyH)(pic)( $\cdot \mathbf{M e C N})$. A number of attempts were made to obtain crystals of this compound from diverse solvents, all attempts which, unlike the recorded methanol crystallisation of ref. ${ }^{23}$, yielded lath-like material which desolvated rapidly resulting in poor quality data. In the event, a data set of useful quality was measured on material obtained from acetonitrile, the latter component remaining ill-defined in the solution/refinement, residing in tunnels evident in the unit-cell projection down $a$, from which it is, presumably, easily lost (Fig. 9). This projection also shows the segregation once again of protonated aza-aromatic and picrate units into separate stacking arrays, in this case with both being infinitely extended and the bipyridinium stacks involving significantly shorter (3.38 $\AA$ ) interplanar distances than those for (2mpH)(pic) (see above), the primary interaction again being the common NH... phenolic-O type (N,H(11)...O(11) 2.779(2), 2.1 $1_{3} \AA$ ). Each stack of units of the one type can be considered to be surrounded by four of the other type, with various interactions linking them. Nitro oxygen to bipyridinium carbon contacts indicate $\mathrm{CH}$...O bonding may be relevant for three of the four sets of neighbours, while for the fourth, a three-coordinate proton, here bridging both bipyridinium- $N$ atoms and phenoxide- $O$, again appears to be involved. Centrosymmetric anion-pair overlaps are found (Fig. S2( $\delta)(i i))$. The fingerprint plot (Fig. 8b)) features all hydrogen bonding and $\pi . . . \pi /$ nitro-based characteristics, but a meaningful comparison with the unsolvated form is prevented by the large diffuse areas caused by the disorder of the acetonitrile component. This indicates that there are significant interactions between the picrate and the solvent, unlike in the case of pyridinium picrate : 1naphthylamine above (PYNPCR) that also suffers from solvent disorder (Figs. 5 c) and $\mathrm{S} 1(\beta)(\mathrm{iii}))$ 


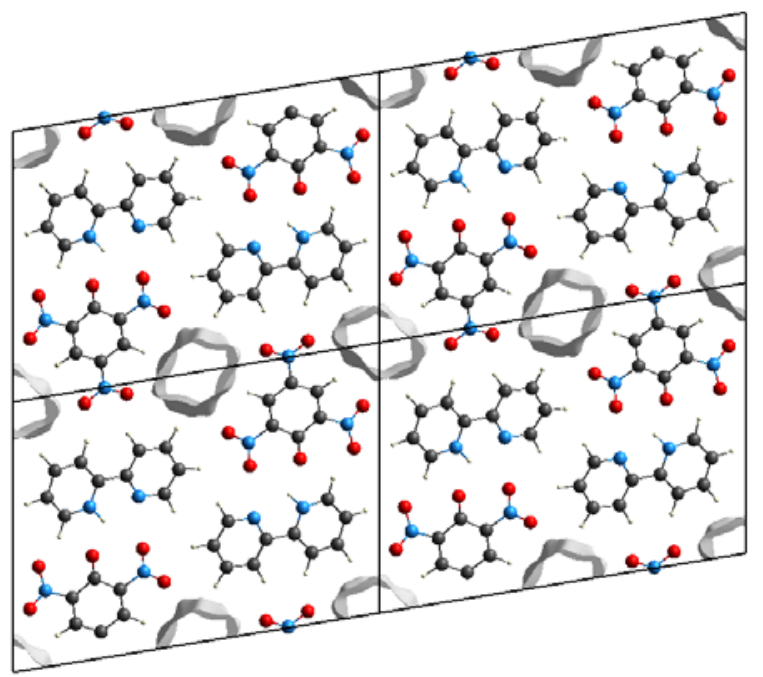

Fig. 9 Void channels (electron-density isovalue $=0.0003$ a.u.) down $a$, in the structure of $(\delta)$ (ii) (bpyH)(pic)(·MeCN), created after removing the disordered solvent molecules from the structure.

(ס) (iii, iv) 4,4'-Bipyridinium bis(picrate) and its monohydrate. Perhaps because of the stability of the mono-protonated cis-configuration of 2,2'-bipyridine ( $c f$. dipyridylamine, anti2,2':6',2"-terpyridine (see below)), we are not aware of any record of a diprotonated cation in a picrate or any other salt, for that matter. Such is not the case with 4,4'-bipyridine where the di-protonated salt 4,4'-bipyridinium bis(picrate), $\left(4,4^{\prime} \mathbf{b p y H}_{2}\right)(\mathbf{p i c})_{2}$ (= bpy' $\left.\left.\mathbf{H}_{2}\right)(\mathbf{p i c})_{2}\right)$, has been recorded, in unsolvated (KAMPIY) ${ }^{24}$ and mono-hydrate (UJOQUF) ${ }^{25}$ forms (Figs. S1( $\delta$ (iii,iv)). Unsurprisingly, in both of these, the principal interactions are those described between picrate phenolic oxygen atoms and the protonated nitrogen atoms at either end of the bipyridyl, associated with pronounced right spikes in the fingerprint plots (Figs. 8 c), d)), significantly more pronounced than in the 2,2'-bipyridinium cases (Figs. 8 a), b)). However, in the hydrated form this spike includes the water molecule interactions, ${ }^{25}$ which here involve 
nitro- rather than phenolic oxygen atoms. Further more distant C-H...O, C-H...N and N-H...N interactions occur in these compounds that are rather unusual among the complete array of compounds investigated. All other approaches and centrosymmetric anion pair overlaps (Fig. S2( $\delta)($ iii,iv)), are clearly governed by very close nitro...nitro interactions, accompanied by more distant nitro... $\pi$ and nitro...phenoxy contacts, with an absence of $\pi \ldots \pi$ interactions. In the unsolvated form, the notably short dichalcogen contacts $\mathrm{O}(2) \ldots \mathrm{O}(4)$ (2.976(2) $\AA$ ) and $\mathrm{O}(5) \ldots \mathrm{O}(6)$ (3.018(3) $\AA$ ) give rise to an intense feature on the fingerprint plot (Fig. 8 c), red) developing into a middle spike. All other nitro-guided interactions are manifest as the characteristic central marker found throughout all compounds. Through hydration of this compound, the middle spike, representative of close O...O contacts, is lost (Fig. 8 d)).

(ס) (v) 2,2':6',2"'-Terpyridinium picrate. Unsurprisingly, stacking is prominent again in the lattice here (Fig. $\mathrm{S} 1(\delta)(\mathrm{v}))$ and it can truly be regarded as an example of a mixed stacking system in that (quasi-) parallel overlaps (with antiparallel dipole arrangements) of tpy...tpy,

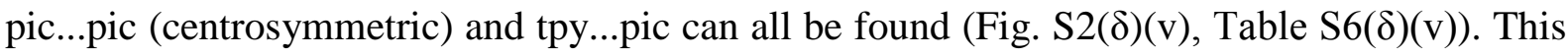
gives rise to a pronounced central $\pi \ldots \pi$ marker on the fingerprint plot (Fig. 8 e)). Although the tpy entities are not truly planar and in the picrate anions some nitro groups show substantial significant rotations out of the ring plane - accompanied by close nitro...nitro and nitro... $\pi$ interactions visible on the fingerprint plot as a second central marker - the mean ring planes of all the aromatic units are quasi-parallel, unlike some situations, e.g., that of the 2,9-dimethyl1,10-phenanthrolinium compound (see below). This is associated also with a different hydrogen-bonding array. Structurally characterized examples of singly protonated tpy units are less common than the doubly protonated form wherein all three nitrogen atoms are syn/cis, the outer pair carrying the protonating hydrogen atoms, these in turn capable of interacting as a chelate with a simple (monatomic) or more complex anion (e.g. $\mathrm{ClO}_{4}, \mathrm{NO}_{3}$, trihaloacetate) 
as the focus, the only singly protonated example with a 'simple' anion being the trifluoromethanesulfonate salt, in which the tpy has one of the peripheral rings (unprotonated) 'anti', as here. ${ }^{14}$ Here, the base provides a further example of the less usual singly protonated form, in the present less usual syn-anti form, presumably a concomitant of intra-cation protonbridging of two N-sites (N,H...N 2.6535(11), 2.25(2) Å) ) (cf. bpy), with further interaction of the proton with picrate involving a contact to a p-nitro oxygen atom $(\mathrm{N}, \mathrm{H}(31) \ldots \mathrm{O}(41)$ 3.108(2), 2.35(2) Å), supported by an adjacent $\mathrm{CH} . . \mathrm{O}$ interaction $(\mathrm{C}, \mathrm{H}(036) \ldots \mathrm{O}(42)$ 3.1666(14), 2.29(2) A) and certainly not to the phenoxy oxygen atom. As adumbrated in the structure of (bpyH)(pic) above, this latter atom and its associated pair of o-nitro oxygen atoms are doubly 'chelated' by pairs of $\mathrm{CH}$ groups on adjacent pyridyl rings $(24,25 ; 33,34)$

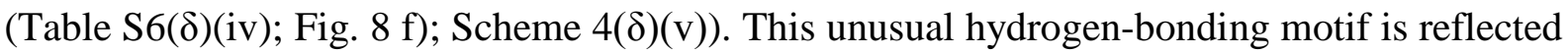
in the fingerprint plot (Fig. 8 e)) by a short right spike in which $\mathrm{C}-\mathrm{H} . . . \mathrm{N}, \mathrm{C}-\mathrm{H} . . . \mathrm{O}$ and $\mathrm{N}-\mathrm{H} . . . \mathrm{O}$ hydrogen bonds are indistinguishable, and by the absence of the left spike. Instead, dihydrogen interactions give rise to a diffuse left/middle spike. The parent base has been recorded in two different polymorphs, ${ }^{48}$ both of which adopt the quasi-planar trans-trans configuration.

\section{(ع) 1,10-Phenanthrolinium picrate and derivative systems}

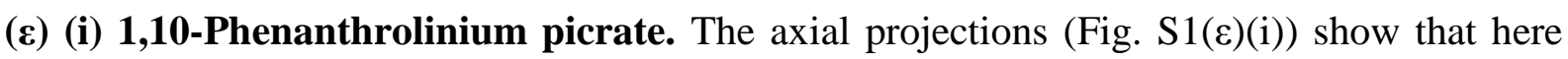
the situation of heterogeneous stacking arises, with phenanthrolinium and picrate entities alternating in the screw-generated stacks (Table S6(E)(i)), the mean aromatic planes being quasi-normal to $b$, with the $\mathrm{C}_{10} \mathrm{~N}_{2} / \mathrm{C}_{6}$ interplanar dihedral angle $3.12(4)^{\circ}$. An ion-pair comprises the asymmetric unit of the structure. The projection of a picrate component upon its adjacent phenanthrolinium counterion involves substantial overlap of ring atoms (and other short contacts) with the greater extent of phenanthroline compared to bipyridine possibly 
favouring hetero-stacking here (Fig. S3( $\delta)(\mathrm{i}))$. There are numerous instances for metal complexes of bpy and phen where stacking arrays are more prominent for the latter. ${ }^{3 \mathrm{c}}$ Two distinct central markers for $\pi \ldots \pi$ as well as nitro-based/ $\pi$...phenoxy interactions are present on the fingerprint plot (Fig. 10 a)). Despite the difference in stacking arrays for phenanthrolinium picrate, however, the hydrogen-bonding between the cation and anion has the usual NH...O(phenoxide) approach (Fig. 2 g)), buttressed by an approach of the adjacent $\mathrm{CH}$ hydrogen atom to an adjacent nitro oxygen atom, these linking adjacent stacks. The other adjacent nitro oxygen atom is devoid of interaction and the corresponding O...O distance is consequently enlarged (Table 2; Fig. 2 g)). That latter nitro-group is also considerably twisted out-of-plane concomitant with a number of close approaches to symmetry-related components, in agreement with the occurrence of a marker in the fingerprint as discussed above. The typical right and left spikes represent the $\mathrm{N}-\mathrm{H} . . . \mathrm{O}$ and $\mathrm{C}-\mathrm{H} . . . \mathrm{O}$ hydrogen bonds. Additionally, a close dihydrogen contact $(\mathrm{H}(5) \ldots \mathrm{H}(024) 2.41(3) \AA ̊)$ features as a quite pronounced middle spike on the fingerprint plot.

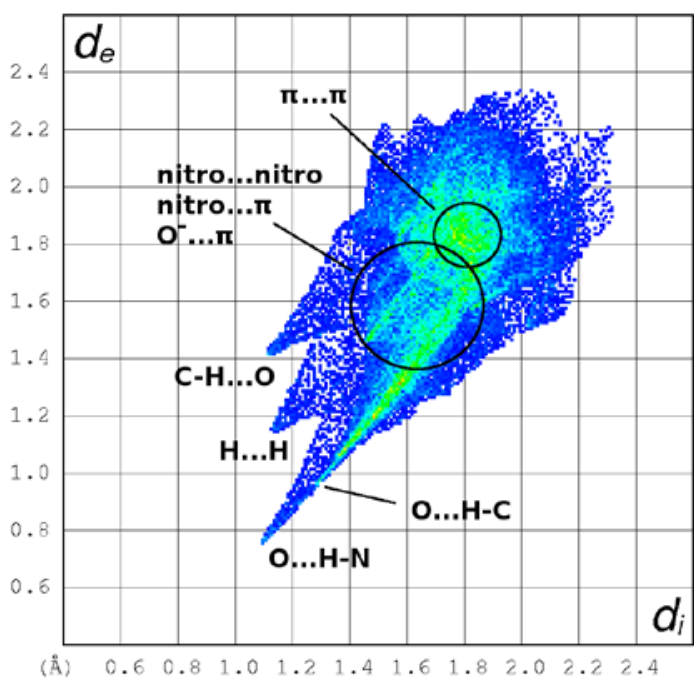

a) $(\varepsilon)(\mathrm{i})(\mathbf{p h e n H})($ pic)

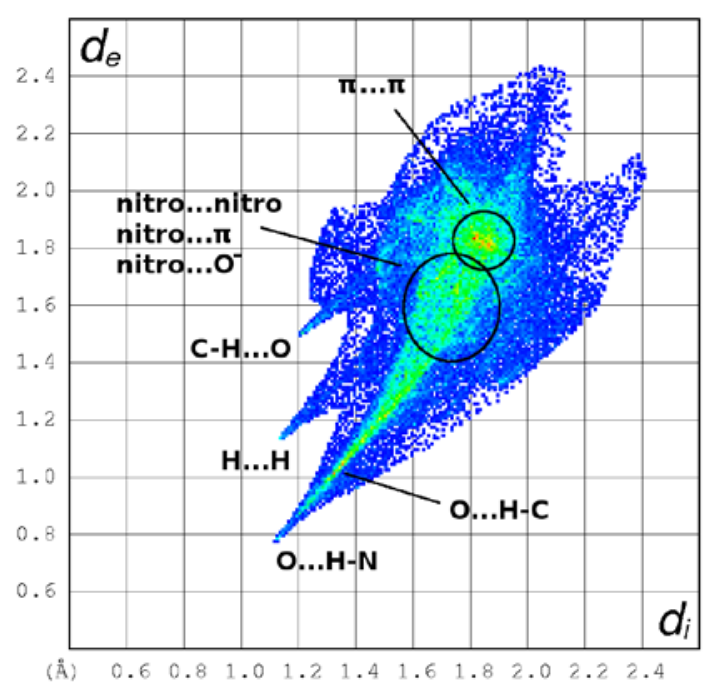

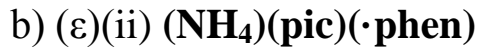




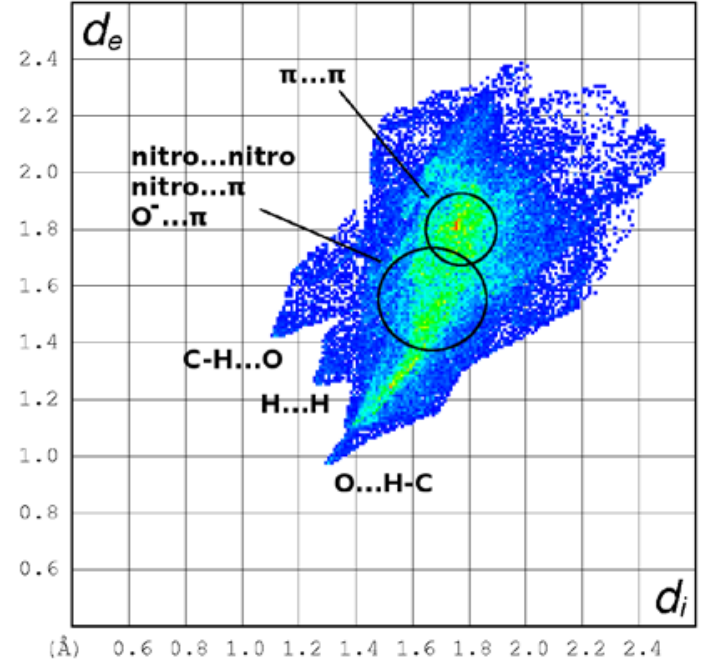

c) $(\varepsilon)\left(\right.$ iii) $\left[(\text { phenH)(bpy'H)(phen)](pic })_{2}\right.$

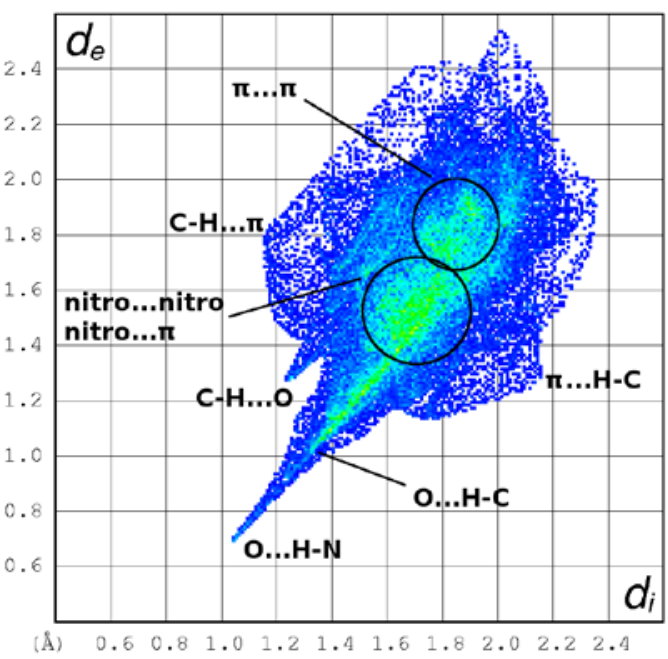

d) (E)(iv) (dmpH)(pic)

Fig. 10 a)-d) Fingerprint plots of group ( $\varepsilon$ ) compounds: phenanthrolinium picrate and derivative systems.

(ع) (ii) Ammonium picrate : 1,10-phenanthroline. In recent studies, we have explored the distribution of protonic hydrogen atoms among salts of mixed bases, notably phen and 'ethylenediamine' ('en'), (and water). ${ }^{17}$ In the present context it is of interest to note a de facto extension of the present study in that vein also, with the report of an adduct of ammonium picrate:phen (1:1) (AMPCPL), ${ }^{26 a}$ an ammonia 'solvate' of (phenH)(pic), $c f$. the hydrates discussed above. However, the protonic hydrogen here is clearly associated with the ammonia species (in contrast to the hydrates where the water molecules of a lesser basicity are not protonated), well removed from the phenanthroline. Here, one pair of the ammonium hydrogen atoms bridge the quasi-coplanar picrate and phenanthroline components $(\mathrm{H}(1) \ldots \mathrm{N}(5,6) 1.89,2.36 ; \mathrm{H}(3) \ldots \mathrm{O}(1,2) 1.99,2.20 \AA)$. Of the other pair, one almost directly out-of-plane, $(\mathrm{H}(2))$, links to an inversion related picrate, that, with its associated phenanthroline and ammonium ion forming the centrosymmetric dimer unit described in

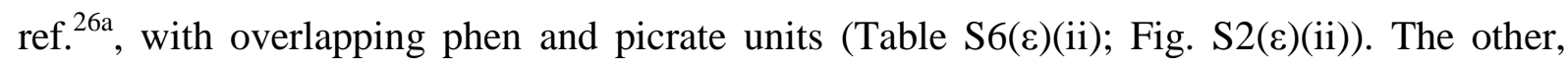
$\mathrm{H}(4)$, closer to the plane, links to the oblique screw-related set evident in the cell projections 
of Fig. S1(E)(ii), with the resultant quasi-columnar arrays shown in the cell projection down $c$. The Hirshfeld surface projection (Fig. $3 \mathrm{f}$ )) emphasizes that the ammonium-phenanthroline pair may be considered a protonated composite here $\left(c f .2[(\mathbf{m o r H})(\mathbf{p i c})]\left(\cdot \mathbf{H}_{2} \mathbf{O}\right)\right.$, Fig. 3 e)), interacting with the quasi-coplanar $\mathrm{O}_{3}$ triad of the picrate ion, with the additional $\mathrm{CH}$... approach. Accordingly, the typical features for $\mathrm{N}-\mathrm{H} . . \mathrm{O}$ and $\mathrm{C}-\mathrm{H} . . . \mathrm{O}$ hydrogen bonds are visible on the fingerprint plot (Fig. 10 b)), with an additional middle spike identifying a close H...H approach. Inversion-related anion...cation overlaps give rise to a distinct $\pi \ldots \pi$ marker, with the usual nitro...nitro, nitro... $\pi$ and nitro...phenoxy contacts also being present.

(ع) (iii) '1,10-Phenanthrolinium 4,4'-bipyridinium 1,10-phenanthroline' bis(picrate). A further complex of interest in this context is '1,10-phenanthrolinium 4,4'-bipyridinium 1,10-phenanthroline' bis(picrate) (INOSUZ) ${ }^{27}$ formulated as '[(phenH)(4,4'-bpyH)(phen)]' (pic) $)_{2}, Z=1$. A reconstitution of the structure has been made, as described in the footnote to Table S6(E)(iii). As modelled in space group $P \overline{1}$, the cation is disposed about an inversion centre located at the mid-point of the central bond of the bipyridyl, one-half of the formula unit comprising the asymmetric unit of the structure. The two nitrogen atoms of the phenanthroline approach that of the pyridyl unit unsymmetrically, the closer distance being 2.7158(4) Å. Associated geometries at these nitrogen atoms are consistent with association of the required protonic hydrogen atom within the pair (rather than the picrate), as modelled in the determination: two components of site occupancy 0.5. Therefore, in the fingerprint plot (Fig. 10 c)), only rather distant C-H...O hydrogen bonds feature, together with a dihydrogen contact, but none of the usual $\mathrm{N}-\mathrm{H}$...O hydrogen bonds. This makes the shape of the fingerprint plot for this compound notably different from any of the others in Fig. 10. Phenanthroline/ium units lie stacked up $a$ in the $a b$ cell face (a 'brick wall' ${ }^{27}$ ), with the bpy'/bpy'H units interleaving centrosymmetrically related pairs of anions, spacing $3.43 \AA$, in a 


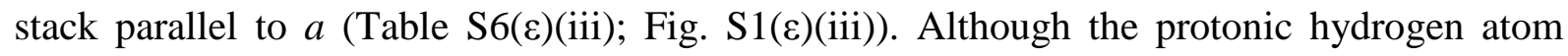
disorder of the model renders a Hirshfeld surface approach uncertain (not, however, impacting on the picrate fingerprint plot), it is evident that the approaches of the picrate to the aromatic components are unusual (Fig. S2(E)(iii)). Out-of-plane twists of the picrate nitro groups result in some less usual nitro-O...nitro-O approaches (Table S6(E)(iii)). These nitro contacts and, separately, $\pi \ldots \pi$ interactions are present in the fingerprint plots as central markers.

(E) (iv) 2,9-Dimethyl-1,10-phenanthrolinium picrate. The introduction of the bulkier methyl substituents onto the phenanthroline skeleton, as in the bridged fusion of a pair of 2mp units yielding 2,9-dimethyl-1,10-phenanthroline, dmp, results in a retreat towards homogeneous stacking arrays. A view of the lattice of this compound (dmpH)(pic) down $a$ (Fig. S2(E)(iv)) shows clearly the segregation of the two types of aromatic entities, the centrosymmetrically related anion pairs overlapping significantly (spacing $3.45 \AA$ ) (Table

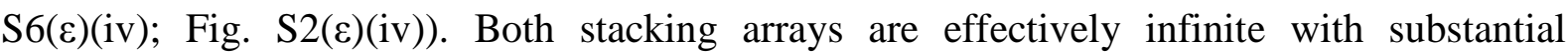
overlaps of the rings in projection and an antiparallel dipole alignment of each unit with respect to the next. In the fingerprint plot (Fig. $10 \mathrm{~d}$ )), a central marker for the $\pi . . . \pi$ interactions is present, but less pronounced and more diffuse than in other cases that exhibit obvious stacking arrangements. Interactions between the stacks/columns appear to involve nitro-O...(H)C contacts in addition to hydrogen-bonding in a manner closely similar to that in the phenanthrolinium compound (again involving three-coordination of the proton by the phenoxide-O and the pair of $\mathrm{HN}$-centres). The orientation required for this hydrogen-bonding, coupled with the steric demands of the methyl groups $(c f .(2 m p y H)(p i c))$, is possibly the reason that the mean planes for the two different stacks are not parallel (interplanar dihedral angle $\left.47.35(4)^{\circ}\right)$. The N,H...O distances (Fig. 2 h)) are 2.719(2), 1.82(3) A. (Structures of the 
forms of the parent base are described in ref. ${ }^{17}$ ). Although there are no C-H ring hydrogen atoms adjacent to the base nitrogen atoms, a close interaction is found between one of the methyl hydrogen atoms, adjacent to the protonating hydrogen atom, and the phenoxide oxygen atom (Tables 2, S6(E)(ii); Fig. 2 h)). The above hydrogen bonds are represented in the fingerprint plot in the usual way through a dominant right spike and a small left spike. A close approach is also found between the other methyl group and the picrate aromatic ring (Table

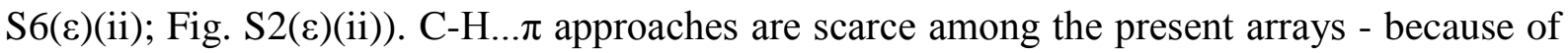
the predominance of interplanar overlaps or, alternatively, because of the nitro-guided interactions - but are apparent in the fingerprint plot in this case. However, nitro...nitro and nitro... $\pi$ interactions are additionally evident in this system, as in all the others.

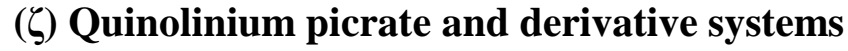

(ל) (i) Quinolinium picrate. Removal of one of the pyridine rings from these phenanthroline systems results in the extended aromatic mono-aza system quinoline, with 2-methylquinoline and 8-hydroxyquinoline derivative thereof and iso-quinoline as its isomer. The crystal structures of the picrate salts of all of these have been described, all with a single ion-pair formula unit comprising the asymmetric unit of the structure. In quinolinium picrate, (quinH)(pic) (UBEGAL), ${ }^{49}$ the cation and anion planes lie quasi-normal to monoclinic $b$

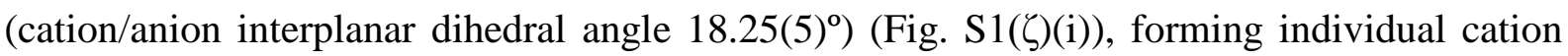
and anion stacks parallel to that axis, with hydrogen bonds between adjacent stacks, linking them into sheets parallel to $b$. Although the homogeneous stacking is clearly evident in the cell projection down $b$, the overlaps between the like components of each stack are rather

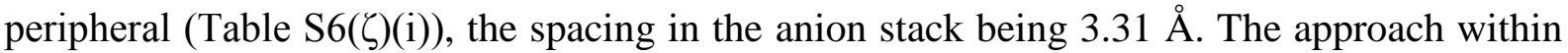
the quasi-coplanar fundamental ion-pair involves the $\mathrm{O}_{3}$ triad of the picrate with the

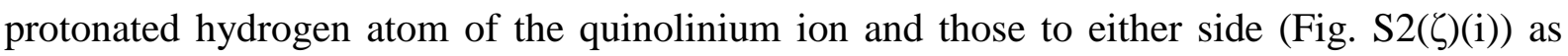


visualised in Figs. 3c) and d) using Hirshfeld-surface projections. In summary, this compound is the archetype of this array of compounds with regards to its intermolecular interaction pattern (cf. Scheme 4). Thus, the fingerprint plot (Fig. 11 a)) shows all of the typical features that present in the other compounds to a greater or lesser degree: Two distinct central markers for nitro-guided interactions and $\pi \ldots \pi$ contacts (even if peripheral and weak in this compound), the strong and short fundamental $\mathrm{N}-\mathrm{H} . . . \mathrm{O}$ hydrogen bond in the right spike together with more distant $\mathrm{C}-\mathrm{H} . . . \mathrm{O}$ and $\mathrm{N}-\mathrm{H} . . . \mathrm{O}$ ones, $\mathrm{C}-\mathrm{H} . . . \mathrm{O}$ interactions with the picrate as donor (left spike), and a middle spike for H...H contacts.

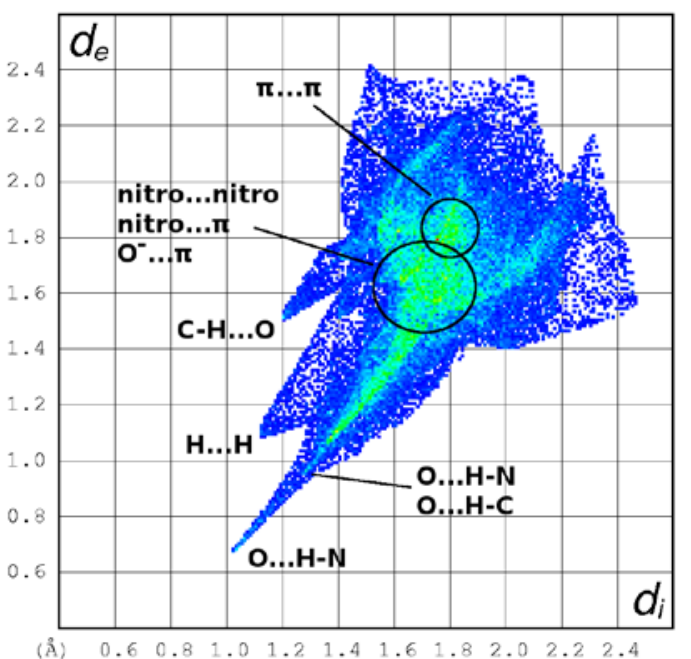

a) (ל)(i) (quinH)(pic)

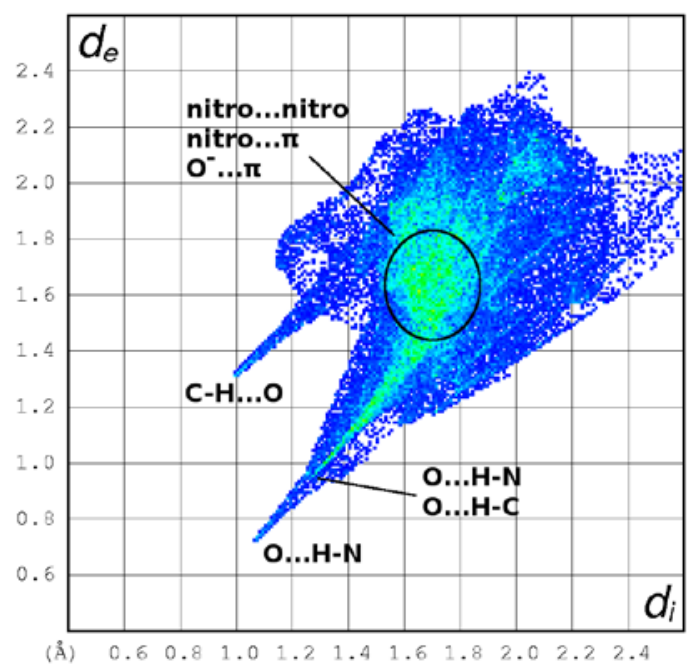

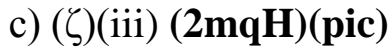

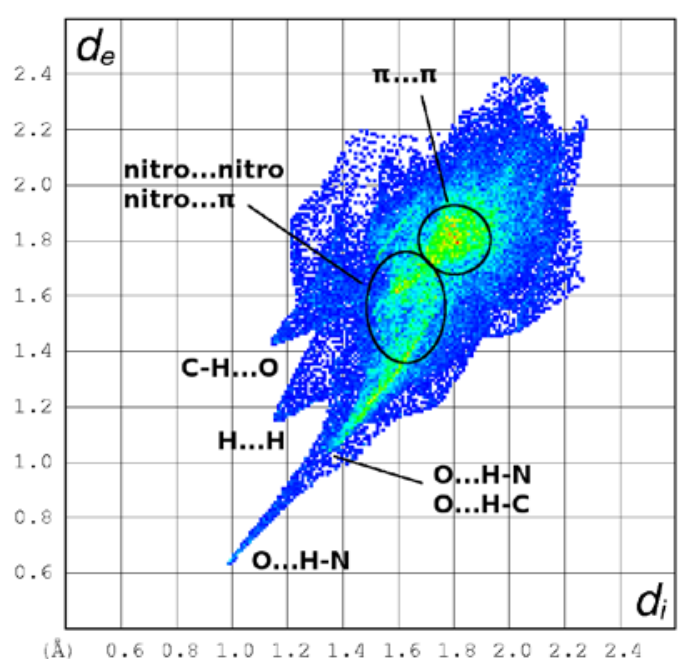

b) (ל)(ii) (iqH)(pic)

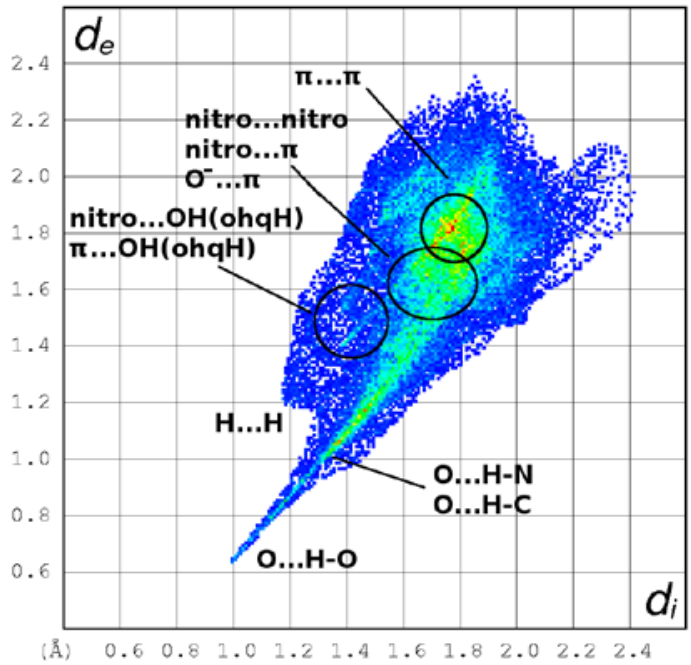

d) (ל)(iv) (ohqH)(pic) 


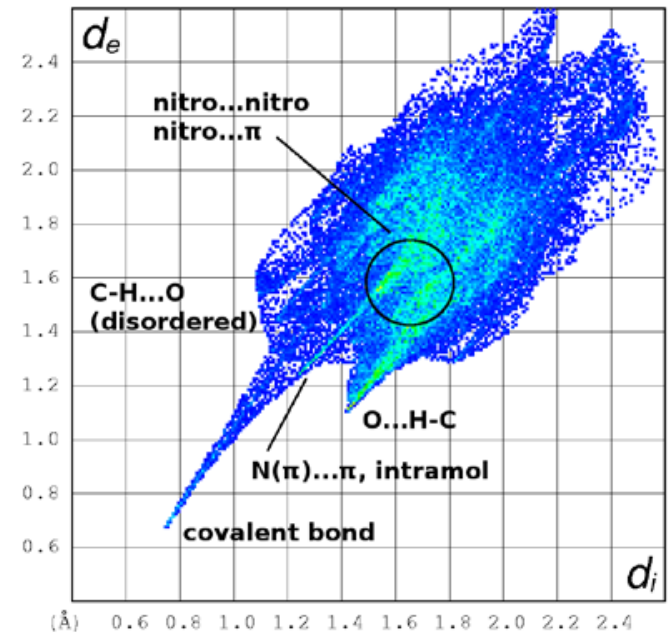

e) $(\zeta)(v)$ (oqpic), picrate component only

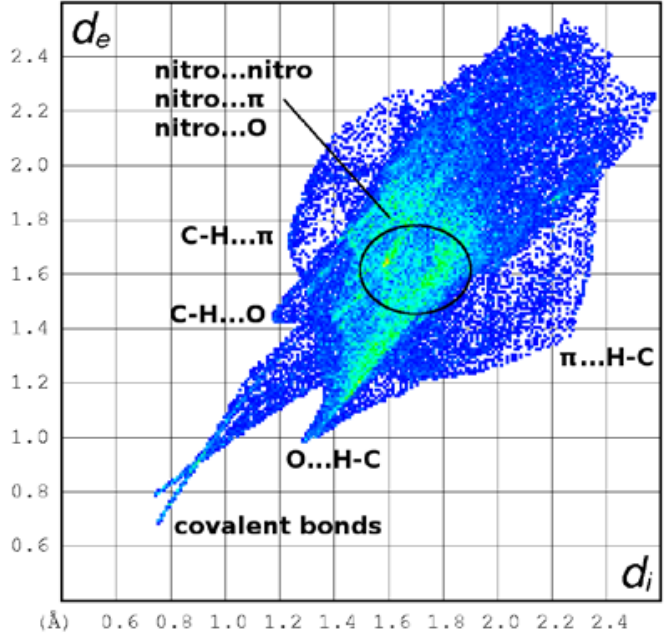

f) $(\zeta)(v i)$ (omppic), picrate component only

Fig. 11. a)-f) Fingerprint plots of group $(\zeta)$ compounds: Quinolinium picrate and derivative systems.

(ל) (ii) iso-Quinolinium picrate. The description of the isomeric iso-quinolinium picrate,

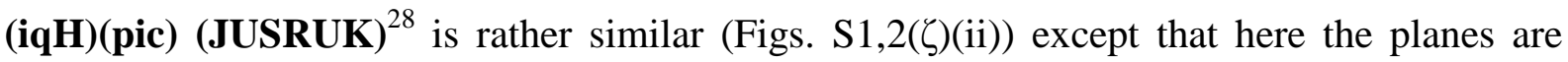
more oblique to $b$ (Fig. $\mathrm{S} 1(\zeta)(\mathrm{ii})$ ), and the stacks have alternating cation and anion members

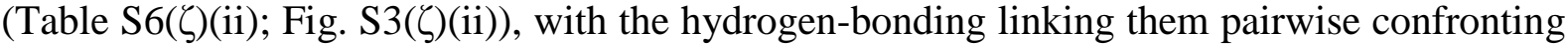
into parallel columns parallel to $b$ and evident in the projection down that axis; significant cation/anion overlaps are found (Fig. S2(ל)(ii)). CH...O support of the NH...O approach comes from the $\mathrm{CH}$ component more distant from the ring junction. Consequently, the fingerprint plot of the isomeric form (Fig. $11 \mathrm{~b}$ )) is very similar to that of quinolinium picrate. The only apparent difference is the much more intense central marker for the $\pi \ldots \pi$ interactions, confirming that the cation/anion overlaps are much more significant here than in the parent quinolinium picrate. $^{50}$ 
(ל) (iii) 2-Methylquinolinium picrate. With substitution of (quinH)(pic) by a 2-methyl group, 2-methylquinolinium picrate, $(\mathbf{2 m q H})(\mathbf{p i c}),{ }^{29}$ (triclinic, $\left.P \overline{1}\right)$ (VATTER), results. Here, as has tended to be the case with other salts of 2-methyl-substituted bases, the anion and cation planes no longer lie parallel to each other. This is readily seen in the cell projection down $a$ (Fig. S1(ל)(iii)), wherein it is clear that the cations lie more nearly quasi-normal to $a$, and may be considered to comprise a sheet parallel to the $a b$ face of the cell (110), such sheets being interleaved with sheets comprised of anions, lying normal to the $b c$ cell diagonal (011). Overlaps between components are rather peripheral, the spacing between centrosymetrically

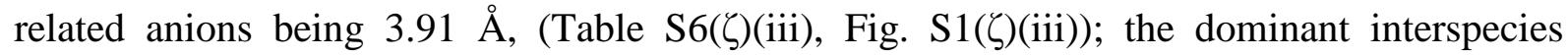
interaction is between protonating and methyl hydrogen atoms and the phenoxide and one $o$ nitro-O atom. The other o-nitro group is twisted markedly out of plane (reflected in unsymmetrical distances in the O...O triad) with both oxygen atoms engaged in quite close approaches to aromatic rings of both types, and assisting engagement with, a pair of inversion-related anions (Fig. S2(ל)(iii)). Therefore, the fingerprint plot (Fig. 11 c)) only shows similarities with those of (iso-)quinolinium picrate (Figs. 11 a), b)) in the spikes for the hydrogen bonds. The central region is completely different in that it presents only a single central marker that represents nitro...nitro, nitro... $\pi$, and $\pi$...phenoxy interactions, with an obvious absence of $\pi \ldots \pi$ interactions.

Comparative non-hydrogen atom geometries are given for the above three (iso-) quinoline species in Table S4, showing little appreciable variation except in the immediate vicinity of the nitrogen atom and any nearby methyl substituent.

(ל) (iv) 8-Hydroxyquinolinium picrate. A further extension, in which the substituent has more far-reaching consequences, and which can itself be competitive in protonation/complexation behaviour, is found in protonated, neutral, de-protonated and 
complexed 8-hydroxyquinoline forms, as recorded in Table S7. In the parent ligand, 'ohq', alone or as a lattice solvate, it is usual to find the $\mathrm{OH}$ hydrogen atom directed toward or even incipiently transferred to the nitrogen atom of a neighbouring molecule forming centrosymmetric dimers. ${ }^{51,52}$ The present complex, prepared for this study, presents a further example of the protonated form, this time as the picrate salt. 8-Hydroxyquinolinium picrate, (ohqH)(pic), crystallizing with one quasi-coplanar ion-pair formula unit as the asymmetric unit of a monoclinic $P 2_{1} / c$ cell. In the cation, both the phenoxy oxygen and aromatic nitrogen atom are protonated. The closest approach to the anion is that of the phenolic hydrogen atom to the phenoxy oxygen atom, with a subsidiary semi-chelate approach to one of the $o$-nitro oxygen atoms, the other $o$-nitro oxygen atom being approached by the ortho HC hydrogen atom (Fig. 2 i)). The NH hydrogen atom exhibits no close approaches within the ion-pair, the Hirshfeld surface projection demonstrating its lack of interaction clearly (Table S6(ל)(iv); Fig.

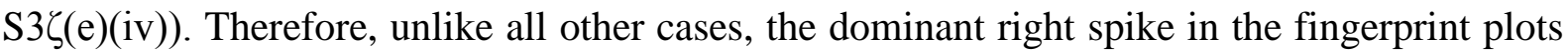
(Fig. 11 d)) refers to the major O-H...O hydrogen bond and not a N-H...O hydrogen bond. More distant $\mathrm{C}-\mathrm{H} . . . \mathrm{O}$ and $\mathrm{N}-\mathrm{H} . . . \mathrm{O}$ hydrogen bonds are visible superimposed on the major spike. However, the C-H groups in the picrate moiety do not act as hydrogen bond donors, so that there is no right spike in the fingerprint plot. Apart from the parameters in the immediate vicinity of the (de-)protonated or coordinating species, the geometries of the aromatic systems remain remarkably constant across the various possibilities exemplified in Table S4.

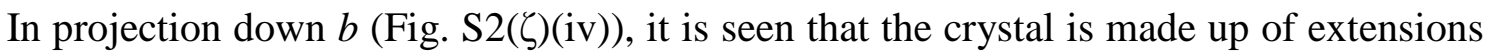
of the ion pair across the $(a c)$ (102) diagonal; consideration of the projection down $(10 \overline{2})$ shows these to comprise ribbons bounded in $b$ with anions and cations alternately overlapping

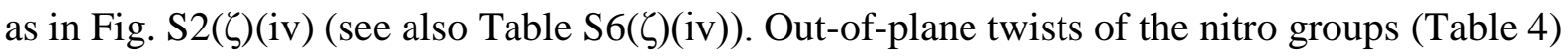
result in a number of close interspecies approaches between the ribbons, evident in the

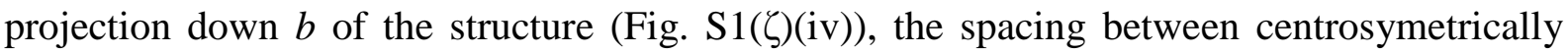


related anions being $3.27 \AA \AA$. They are present as a lower marker on the fingerprint plot and include approaches of the oxygen atom of the hydroxyl group and other $\pi$-systems or nitro groups. Significant $\pi . . . \pi$ interactions feature as a distinct upper central marker, arising from inversion-related anion....anion and anion...cation overlaps. The development of a rather diffuse middle/left spike indicates H...H approaches.

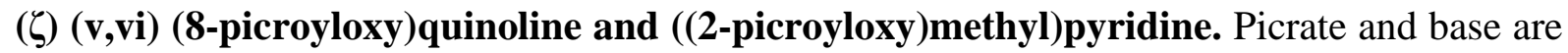
unusually combined in interesting extensions of this system as found in structurally characterized (8-picroyloxy)quinoline (oqpic) (JOKTOS), notionally 'precursive' of the cyclized form found in the counterpart Meisenheimer complex picroyloxy)methyl)pyridine (omppic) (JOKTIM), ${ }^{30}$ (Figs. $\mathrm{S} 1(\zeta)(\mathrm{v}, \mathrm{vi})$ ); each has one molecule comprising the asymmetric unit of the structure. In the former, the 'phenolic' C-O distance is appreciably longer than those in the acid or salts presently described (Table 2), and the two components of the structure essentially act independently in the manner described above for the other quinoline picrate compounds. It is evident from the cell and overlap

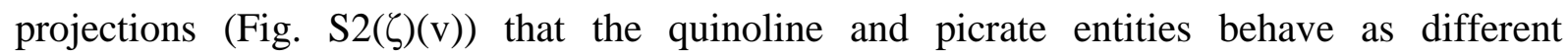
components of the structure, the former lying in a parallel stack up $c$, in the cell projection down $b$, while the picrate groups, oriented more nearby normal to $b$, overlap and form interleaving sheets parallel to the $b c$ face, spacing $3.46 \AA$ A. Contacts between symmetry related picrate groups by way of out-of-plane nitro orientations are evident. The presence of nitrogroup disorder hampers Hirshfeld surface analysis, but we have provisionally generated a fingerprint plot of the picrate entity only (Fig. 11 e)) to enable comparability and compatibility with the other picrate plots. Disregarding the long spike for the covalent C-O bond, the following features, also typical of the unbound picrates, are visible: C-H...O hydrogen bonds (right and left spikes) as well as nitro...nitro and nitro... $\pi$ interactions, with a 
clear lack of $\pi \ldots \pi$ contacts of the picrate unit. However, intramolecular interaction between $\mathrm{N}(1)$ in the quinoline moiety with the picrate $\pi$-system is evident as a thin but intense middle spike superimposing on that of the covalent bond.

In the actual Meisenheimer complex, JOKTIM, where the independence of the two major components is lost (intramolecular interplanar $\mathrm{C}_{5} \mathrm{~N}(\mathrm{ar}) / \mathrm{C}_{6}\left(\mathrm{ar} \mathbf{r}^{\prime}\right)$ dihedral $\left.89.48(9)^{\circ}\right)$, interactions are more complex, although the formation of sheets parallel to $b$, with intersheet nitro-O...nitro-O contacts, is evident (Table S6(ל)(vi); Fig. $\mathrm{S} 1(\zeta)(\mathrm{vi}))$, the nitro groups again being twisted well out-of-plane; the inter-picrate spacing is $3.51 \AA$. The fingerprint plot of the picrate unit only (Fig. $11 \mathrm{f}$ )) is quite similar to the one for JOKTOS in that C-H...O hydrogen bonds and nitro...nitro interactions are identifiable, with a clear absence of $\pi . . . \pi$ interactions. However, faint and diffuse features indicating distant C-H... $\pi$ interactions have appeared. Interestingly, the development of the spike describing the interaction between $\mathrm{N} 1$ and the picrate system in the fingerprint plot of JOKTOS has now developed into a second spike for a covalent $\mathrm{C}-\mathrm{N}$ bond, but maintaining the direction and principal position of the spike.

\section{Conclusions}

Single crystal X-ray studies have defined the structures of a number of crystalline pricrate salts with the monoprotonated aromatic nitrogen bases pyridine, 2-methylpyridine, 2,2'bipyridine, 2,2':6',2"-terpyridine, 1,10-phenanthroline, 2,9-dimethyl-1,10-phenanthroline, bis(2-pyridyl)-amine and 8-hydroxyquinoline, augmented by studies of those of the saturated cyclic counterparts piperidine and morpholine, and systematically extending previous studies recording salts of aliphatic and related base salts. In company with related literature systems, the nature of interactions within the crystal have been explored, assisted by the use of the Hirshfeld surface approach. The analysis of Hirshfeld-surface derived fingerprint plots is shown to be a valuable tool to facilitate the identification of different types of intermolecular 
interactions and their relative significance with respect to each other. In one of the most extended comparisons of this kind to date, different crystalline environments for the same molecular species (the picrate ion) give rise to a remarkable variety of features, while still maintaining the central motifs in most of them. In almost all cases, the fundamental motif of the structure is a quasi-coplanar cation...anion pair, the N-protonating hydrogen atom of the cation approaching the phenoxy-oxygen of the anion; the latter, in conjunction with the quasicoplanar nitro-groups to either side, is capable of acting in a quasi-tridentate O...O...O mode, and the principal interaction of the $\mathrm{NH}$ component, unsymmetrically chelated between an O...O pair, may be buttressed by an often more significant interaction of the third oxygen atom with a $\mathrm{CH}$ hydrogen atom of the base ortho to the nitrogen atom. A notable exception to the above description is found in the literature report of the unsolvated 2,2'-bipyridinium salt, wherein the protonating $\mathrm{NH}$ hydrogen atom approaches the oxygen atoms of the picrate group, consistent with the presence of negative charge contingent on the presence of significant quinonoid resonance contribution. The latter, together with the bonding in the parent picric acid, are explored theoretically confirming the importance of this resonance form in the picrate salts. The (co-) planar components are found to stack in most cases, although Hirshfeld surface analysis suggests interaction between the individual atom components of the planes to be feeble, albeit extensive, relative to those of the $\mathrm{NH}$...O grouping. Where the aromatic base carries a 2-methyl substituent ortho to the nitrogen atom, the above interaction is thwarted and the aromatic base and picrate components lie oblique to each other; where the base ring is saturated, the structures are dominated by picrate stacks alone, surrounded by sometimes interesting base dispositions. Furthermore, nitro...nitro as well as nitro... $\pi$ contacts occurring at the picrate peripheries are significant motifs that lead to remarkably short O...O or $\mathrm{N}$...O contacts and may be at least as important as $\pi$-stacking. 


\section{Acknowledgments}

We gratefully acknowledge the assistance of Dr Adam I. Stash of the Karpov Institute, Moscow, in accessing relevant Russian data. We also thank Prof. Zilu Chen (Guangxi Normal University, Guilin 541004, PRC) who kindly made available the coordinates of INOSUZ ${ }^{27}$. Simon Grabowsky acknowledges financial support by the Australian Research Council within the Discovery Projects DP110105347 and DP130103488.

\section{References}

(1) (a) M. A. Bredig and H. Moller, Z. Kristallogr., Kristallgeom., Kristallphys., Kristallchem., 1929, 71, 331; (b) E. N. Duesler, J. H. Engelmann, D. Y. Curtin and I. C. Paul, Cryst. Struct. Comm., 1978, 7, 449 (CCDC: PICRAC, 295 K); (c) T. Srikrishnan, M. Soriano-Garcia and R. Parthasarathy, Z. Krist., 1980, 151, 317 (PICRAC11, '283-303' K (seemingly the CCDC interpretation of unspecified or 'room'-temperature)); (d) M. SorianoGarci, T. Srikrishnan and R. Parthasarathy, Acta Crystallogr., Sect. A, 1978, 34, S114 (PICRAC01, '283-303' K (no deposited coordinates)); (e) L. Yang, T.-L. Zhang, C.-G. Feng, J.-G. Zhang and K.-B. Yu, Hanneng Cailiao, 2001, 9, 37 (no CCDC entry); (f) B. Narayana, B. K. Sarojini, H. S. Yethirajan and M. Bolte, CCDC Private Communication, 2007 (PICRAC12, 173 K); (g) V. Bertolasi, P. Gilli and G. Gilli, Cryst. Growth Des., 2011, 11, 2724 (PICRAC13, 120 K).

(2) (a) C. L. Perrin, Pure Appl. Chem., 2009, 81, 571; (b) P. Gilli, L. Pretto, V. Bertolasi and G. Gilli, Acc. Chem. Res., 2009, 42, 33; (c) T. Steiner, Angew. Chem. Int. Ed., 2002, 41, 48.

(3) (a) C. Janiak, J. Chem. Soc., Dalton Trans., 2000, 3885; (b) W. B. Jennings, B. M. Farrell and J. F. Malone, Acc. Chem. Res., 2001, 34, 885; (c) J. M. Harrowfield, J. Chem. Soc., Dalton Trans., 1996, 3165 (and references therein); (d) L. Loots and L. J. Barbour, 
Cryst. Eng. Comm., 2012, 14, 300; (e) S. Ehrlich, J. Moellmann and S. Grimme, Acc. Chem. Res., 2013, 46, 916.

(4) (a) D. A. Dougherty, Acc. Chem. Res., 2013, 46, 885; (b) H. T. Chifotides and K. R. Dunbar, Acc. Chem. Res., 2013, 46, 894; (c) M. M. Watt, M. S. Collins and D. W. Johnson, Acc. Chem. Res., 2013, 46, 955.

(5) (a) (benzene) H. Takayanagi, Y. Toubai, M. Goto, S.-I. Yamaguchi and H. Ogura, Chem. Pharm. Bull., 1991, 39, 2491 (ZZZAGV01, 296 K); (b) (naphthalene) A. Banerjee and C. J. Brown, Acta Crystallogr., Sect. C, 1985, 41, 82 (PVVBHJ01, 293 K); (c) (anthracene) F. H. Herbstein and M. Kaftory, Acta Crystallogr., Sect. B, 1976, 32, 387 (ANTPIC, '283303' K); (d) (phenanthrene) S. Yamaguchi, M. Goto, H. Takayanagi and H. Ogura, Bull. Chem. Soc. Jpn., 1988, 61, 1026 (PVVBGS01, 296 K). (Preliminary surveys are recorded in (e) (benzene) F. H. Herbstein, M. Kaftory and H. Regev, J. Appl. Cryst., 1976, 9, 361 (ZZZAGV); (f) (benzene, naphthalene) F. H. Herbstein and M. Kaftory, Acta Crystallogr., Sect. B, 1975, 31, 60 (PVVBHJ).)

(6) H. Takayanagi, M. Goto, K. Takeda and Y. Osa, Yakugaku Zasshi, 2004, 124, 751 (pyridinium, quinolinium, iso-quinolinium picrates) (no CCDC files available, see ref. ${ }^{11}$ (PYRPICXX); refs. ${ }^{8,49}$ (UBEGAL); ref. $^{28}$ (JUSRUK)).

(7) K. Honda, H. Yamawaki, M. Matsukawa, M. Goto, T. Matsunaga, K. Aoki, M. Yoshida and S. Fujiwara, Acta Crystallogr., Sect. C, 2003, C59, m319 (EJELIO).

(8) V. Stilinovic and B. Kaitner, Cryst. Growth Des., 2011, 11, 4110 (UBEXXX (18 CCDC entries; UBEGAL relevant to the present); IPOZAO01, KABKIJ02 (all 295 K)).

(9) H. Takayanagi, T. Kai, S.-I. Yamaguchi, K. Takeda and M. Goto, Chem. Pharm. Bull., 1996, 44, 2199 (REYDXX (with ZZZLBS01, 6 CCDC entries)). 
(10) Ref. $^{1 \mathrm{~g}}$ (OWIXXX (10 CCDC entries); KABKIJ01, KUVLAP01, GISDES01, KIKLEW01 (all 'room-temperature' ('283-303'), except PICRAC13 (120K), relevant to the present)).

(11) (a) A. N. Talukdar and B. Chaudhuri, Acta Crystallogr., Sect. B, 1976, 32, 803 (PYRPIC; monoclinic, '283-303' K); (b) H. Takayanagi, R. Kawaoka, K. Chin, M. Goto, S.-I. Yamaguchi and H. Ogura, Anal. Sci., 1990, 6, 321 (PYRPIC01, monoclinic '283-303' K); (c) M. Botoshansky, F. H. Herbstein and M. Kapon, Acta Crystallogr., Sect. B, 1994, 50, 191 (PYRPIC02, monoclinic, 298 K (correcting PYRPIC (11a)); PYRPIC03, triclinic, 298K); (d) K. Kulicki, Biul. Wojsk. Akad. Tech. Jar. Dabrow., 1967, 16, 91 (no CCDC entry); (e) A. Kofler, Z. Elektrochem., 1944, 50, 200 is an early report noting the existence of two phases.

(12) E. J. Chan, J. M. Harrowfield, B. W. Skelton and A. H. White, Inorg. Chim. Acta, to be submitted.

(13) V. Russell, M. Scudder and I. Dance, J. Chem. Soc., Dalton Trans., 2001, 789.

(14) (a) P. C. Junk, C. J. Kepert, L. I. Semenova, B. W. Skelton and A. H. White, Z. Anorg. Allg. Chem., 2006, 632, 1293; (b) A. Hergold-Brundić, Z. Popović and D. MatkowičČalogović, Acta Crystallogr., Sect. C, 1996, 52, 3154 (the triflate).

(15) P. C. Junk, W.-M. Lu, L. I. Semenova, B. W. Skelton and A. H. White, Z. Anorg. Allg. Chem., 2006, 632, 1303.

(16) Effendy, P. C. Junk, C. J. Kepert, L. M. Louis, T. C. Morien, B. W. Skelton and A. H. White, Z. Anorg. Allg. Chem., 2006, 632, 1312.

(17) (a) J. H. Buttery, Effendy, P. C. Junk, S. Mutrofin, B. W. Skelton, C. R. Whitaker and A. H. White, Z. Anorg. Allg. Chem., 2006, 632, 1326. Other reports of the two 'polymorphs' of 2,9-dimethyl-1,10-phenanthroline are recorded in (b) D. K. Sen, Acta Crystallogr., Sect. B, 1969, 25, 988 (tetragonal, space group $\left(I 4_{1} / a\right)$ and cell only); (c) S. Baggio, R. Baggio and A. W. Mombru, Acta Crystallogr., Sect. C, 1998, 54, 1900 (the 
monoclinic C2/m dihydrate); (d) D. Britton, L. C. Thompson and R. C. Holz, Acta Crystallogr., Sect. C, 1991, 47, 1101 (the tetragonal ( $\left.I 4_{1} / a\right)$ (cf. (b)) hemihydrate).

(18) P. C. Junk, Y. Kim, B. W. Skelton and A. H. White, Z. Anorg. Allg. Chem., 2006, 632, 1340.

(19) A. Szumna, J. Jurczak and Z. Urbańczyk-Lipkowska, J. Mol. Struct., 2000, 526, 165.

(20) J. Bernstein, H. Regev and F. H. Herbstein, Acta Crystallogr., Sect. B, 1980, 36, 1170 (PYNPCR).

(21) K. Saminathan, C. Muthamizhchelvan and K. Sivakumar, Acta Crystallogr., Sect. E, 2005, 61, o4379 (VAZJAI, 293 K).

(22) (a) N. Vembu and F. R. Fronczek, Acta Crystallogr., Sect. E, 2009, 65, o516 (KOMTUC, 90.0(5) K); (b) M. S. Refat, L. A. El-Zayat and O. Z. Yeşilel, Spectrochim. Acta, 2010, A75, 745 (KOMTUC01, 293(2) K). An early brief and rather mysterious allusion to two phases of this compound (and also the pyridinium salt) is found in (c) J. Mitchell and W. M. D. Bryant, J. Am. Chem. Soc., 1943, 65, 128.

(23) S. J. Kavitha, K. Panchanatheswaran, J. N. Low, G. Ferguson and C. Glidewell, Acta Crystallogr., Sect. C, 2006, 62, 0165 (UCOFUO, 120 K).

(24) L.-F. Ma, B.-T. Zhao and L.-Y. Wang, Acta Crystallogr., Sect. E, 2005, 61, 0964 (KAMPIY, 293 K).

(25) T. Akutagawa, T. Uchimaru, K.-I. Sakai, T. Hasegawa and T. Nakamura, J. Phys. Chem. B, 2003, 107, 6248 (UJOQUF, 296 K).

(26) (a) C. L. Jones, G. H. Milburn, L. Sawyer and D. L. Hughes, Acta Crystallogr., Sect. B, 1981, 37, 1548 (AMPCPL, '283-303' K); (b) R. K. Tiwari, C. Chandrakumar and T. P. Singh, Z. Kristallogr., 1981, 156, 245 (AMPCPL01, '283-303' K; no coords. available); (c) 
R. K. Tiwari, V. V. Joshi, T. P. Singh and N. S. Poonia, Ind. J. Phys. A, 1982, 56, 381 (AMPCPL11, '283-303' K).

(27) Z. Chen, F. Liang, R. Hu, H. Liang and K. Yu, Chem. J. Internet, 2001, 3, 10 (INOSUZ, $295 \mathrm{~K}$; no deposited coordinates. Correspondence with Professor Chen has enabled reconstitution of a .CIF file from the supplied set of non-hydrogen atom coordinates; see Table S6( $\delta)(\mathrm{iv}))$.

(28) M. Goto, H. Takayanagi, K. Furuhata, H. Ogura, K. Saito, K. Sugai and N. Sugiyama, Chem. Pharm. Bull., 1992, 40, 1612 (JUSRUK, '283-303' K).

(29) S. Jin, X.-H. Lu, D. Wang and W. Chen, J. Mol. Struct., 2012, 1010, 17 (VATTER, 298 K).

(30) V. N. Knyazev, V. N. Drozd, N. S. Patalakha, D. S. Yufit and Y. T. Struchkov, Zh. Org. Khim., 1991, 27, 192 (JOKTIM, JOKTOS, 295 K).

(31) (a) M. A. Spackman and D. Jayatilaka, Cryst. Eng. Comm., 2009, 11, 19; (b) S. K. Wolff, D. J. Grimwood, J. J. McKinnon, M. J. Turner, D. Jayatilaka and M. A. Spackman, Crystal Explorer (Version 3.0), University of Western Australia, 2010; (c) M. J. Turner, J. J. McKinnon, D. Jayatilaka and M. A. Spackman, Cryst. Eng. Comm., 2011, 13, 1804.

(32) J. J. McKinnon, D. Jayatilaka and M. A. Spackman, Chem. Commun., 2007, 3814.

(33) M. A. Spackman and J. J. McKinnon, Cryst. Eng. Comm., 2002, 4, 378.

(34) J. J. McKinnon, F. P. A. Fabbiani and M. A. Spackman, Cryst. Growth Des., 2007, 7, 755 .

(35) J. Bernstein, Cryst. Growth Des., 2011, 11, 632.

(36) G. M. Sheldrick, Acta Crystallogr., Sect. A, 2008, 64, 112.

(37) K. Anitha, S. Athimoolam and S. Natarajan, Acta Crystallogr., Sect. C, 2006, 62, o567 (PEMCUG, 293 K).

(38) A. L. Spek, J. Appl. Cryst., 2003, 36, 7. 
(39) (a) C. C. Carvalho, A. J. Camargo, M. Y. Teijido, P. C. Isolani, G. Vicentini and J. Zukerman-Schpector, Z. Krist., 2003, 218, 575 (GAHHUT, 293 K); (b) V. K. Kumar and R. Nagalakshmi, Spectrochim. Acta, 2007, A66, 924 (GAHHUT01, '283'-303' K) (no coords.).

(40) Gaussian 03, Revision E.01, M. J. Frisch, G. W. Trucks, H. B. Schlegel, G. E. Scuseria, M. A. Robb, J. R. Cheeseman, J. A. Montgomery, T. Vreven, K. N. Kudin, J. C. Burant, J. M. Millam, S. S. Iyengar, J. Tomasi, V. Barone, B. Mennucci, M. Cossi, G. Scalmani, N. Rega, G. A. Petersson, H. Nakatsuji, M. Hada, M. Ehara, K. Toyota, R. Fukuda, J. Hasegawa, M. Ishida, T. Nakajima, Y. Honda, O. Kitao, H. Nakai, M. Klene, X. Li, J. E. Knox, H. P. Hratchian, J. B. Cross, V. Bakken, C. Adamo, J. Jaramillo, R. Gomperts, R. E. Stratmann, O. Yazyev, A. J. Austin, R. Cammi, C. Pomelli, J. W. Ochterski, P. Y. Ayala, K. Morokuma, G. A. Voth, P. Salvador, J. J. Dannenberg, V. G. Zakrzewski, S. Dapprich, A. D. Daniels, M. C. Strain, O. Farkas, D. K. Malick, A. D. Rabuck, K. Raghavachari, J. B. Foresman, J. V. Ortiz, Q. Cui, A. G. Baboul, S. Clifford, J. Cioslowski, B. B. Stefanov, G. Liu, A. Liashenko, P. Piskorz, I. Komaromi, R. L. Martin, D. J. Fox, T. Keith, M. A. AlLaham, C. Y. Peng, A. Nanayakkara, M. Challacombe, P. M. W. Gill, B. Johnson, W. Chen, M. W. Wong, C. Gonzalez and J. A. Pople, Gaussian, Inc., Wallingford CT, 2004.

(41) A. E. Reed, L. A. Curtiss and F. Weinhold, Chem. Rev., 1988, 88, 899.

(42) E. D. Glendening, A. E. Reed, J. E. Carpenter and F. Weinhold, NBO, Version 3.1.

(43) (a) Z. Zimpel, B. R. Nelson and J. A. Weil, Can. J. Chem., 1996, 74, 70; (b) P. C. Chen and C. C. Huang, J. Mol. Struct. (Theochem), 1993, 282, 287; (c) B. M. Rice, S. Sahu and F. J. Owens, J. Mol. Struct. (Theochem), 2002, 583, 69.

(44) (a) P. Srinivasan, T. Kanagasekaran and R. Gopalakrishnan, Cryst. Growth Des., 2008, 8, 2340; (b) R. M. Claramunt, C. Lopez and J. Elguero, J. Heterocyclic Chem., 1999, 36, 595; (c) P. Srinivasan, T. Kanagasekaran and R. Gopalakrishnan, Spectrochim. Acta, 
2008, A71, 592; (d) J. P. Jasinski, R. J. Butcher, Q. N. M. H. Al-Arique, H. S. Yathirajan and B. Narayana, Acta Crystallogr., Sect. E, 2009, 65, o1738; (e) J. P. Jasinski, R. J. Butcher, M. T. Swamy, H. S. Yathirajan and A. R. Ramesha, Acta Crystallogr., Sect. E, 2009, 65, o2788.

(45) J. M. Harrowfield, B. W. Skelton and A. H. White, Aust. J. Chem., 1995, 48, 1311.

(46) (a) Y. H. Lee, Y. C. Park, J. M. Harrowfield, P. Thuéry and Y. Kim, Bull. Kor. Chem. Soc., 2007, 28, 1429; (b) J. M. Harrowfield, G. H. Sharverdizadeh and A. A. Soudi, Supramol. Chem., 2003, 15, 367; (c) P. M. Zorky and O. V. Grineva, J. Inc. Phen. Macr. Chem., 2004, 48, 81; (d) M. E. Brezgunova, J. Lieffrig, E. Aubert, S. Dahaoui, P. Fertey, S. Lebègue, J. G. Ángyán, M. Fourmigué and E. Espinosa, Cryst. Growth Des., 2013, 13, 3283.

(47) (a) J. E. Johnson and R. A. Jacobsen, Acta Crystallogr., Sect. B, 1973, 29, 1669 (orthorhombic, Pccn, Z = 4, 297 K); (b) H. Schödel, C. Näther, H. Bock and F. Butenschön, Acta Crystallogr., Sect. B, 1996, 52, 842 (monoclinic, $P 2_{1} / c, Z=8,150$ K); (c) G. J. Pyrka and A. A. Pinkerton, Acta Crystallogr., Sect. C, 1992, 48, 91 (triclinic, $P \overline{1}, Z=$ 4, 296 K).

(48) (a) C. A. Bessel, R. F. See, D. L. Jameson, M. R. Churchill and K. J. Takeuchi, J. Chem. Soc., Dalton Trans., 1992, 3223 (orthorhombic, $P 22_{1} 2_{1} 2_{1}, Z=4,295$ K); (b) K. F. Bowes, I. P. Clark, J. M. Cole, M. Gourlay, A. M. E. Griffin, M. F. Mahon, L. Ooi, A. W. Parker, P. R. Raithby, H. A. Sparkes and M. Towrie, Cryst. Eng. Comm., 2005, 269 (monoclinic $P 2{ }_{1} / c, Z=8,100,150,200,250 \mathrm{~K}$ ).

(49) (a) see ref. ${ }^{8}$ (UBEGAL, '283-303' K); (b) a different polymorph of the compound is also described in: M. Goto, G. Takayanagi, K. Furuhata, H. Ogura, K.-I. Saito, K. Sugai and N. Sugiyama, Anal. Sci., 1992, 8, 579 (no CCDC entry (coords. supplied in paper (T?))).

(50) C. A. Hunter and J. K. M. Sanders, J. Am. Chem. Soc., 1990, 112, 5525.

(51) The following have associated published or deposited CCDC coordinate files: (a) P. Roychowdhury, B. N. Das and B. S. Basak, Acta Crystallogr., Sect. B, 1978, 34, 1047 (HXQUIN10, '283-303' K); (b) T. Banerjee and N. N. Saha, Acta Crystallogr., Sect. C, 1986, 
42, 1408 (HXQUIN03, 297 K); (c) J. Zhang and L. Wu, CCDC Private communication, 2005 (HXQUIN11, '283-303' K); (d) P. G. Jones, CCDC Private communication, 2012 (HXQUIN13, 100K); (e) The report of V. Krishnakumar, R. Nagalakshmi and P. Janaki, Spectrochim. Acta, 2005, A61, 1097 (HXQUIN12, '283-303' K) carries no deposited coordinates, reporting the space group as $P 2_{1} / m$ rather than $F d d 2$.

(52) D. L. Hughes and M. R. Truter, J. Chem. Soc., Dalton Trans., 1979, 520 (KHQUIN, KHQUDQ, '283-303' K (both)). 